Supporting Information For:

\title{
A Cyclic Ruthenium Benzylidene Initiator Platform Enhances Reactivity for Ring- Expansion Metathesis Polymerization
}

Teng-Wei Wang, Pin-Ruei Huang, Jayme L. Chow, Werner Kaminsky and Matthew R. Golder*

Department of Chemistry and Molecular Engineering \& Science Institute,

University of Washington, Seattle, WA 98195, United States

Corresponding author: goldermr@uw.edu

\section{Table of Contents}

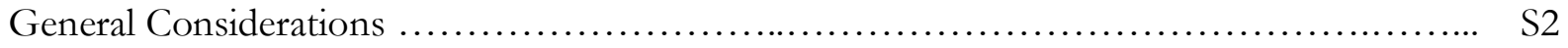

Synthetic and Experimental Procedures......................................... S3

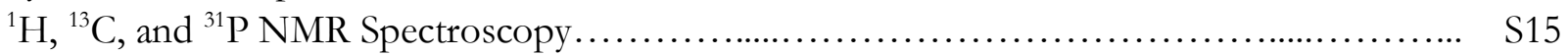

X-ray Crystallography ........................................................... S28

Solution State Stability .................................................. S48

Computational Strain Energy Analysis............................................ S48

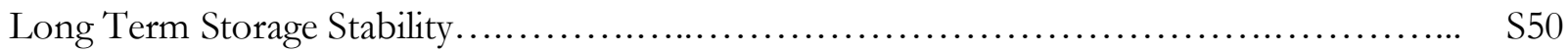

Additional GPC-MALS-IV Data............................................ S52

References.............................................................. S57

Raw NMR Kinetics Data..................................................... S58

DFT Coordinates ................................................................ S62 


\section{General Considerations}

\section{Materials}

All reagents were purchased from commercial suppliers and used as received unless otherwise noted. Glassware was flame dried or dried in an oven overnight at $120{ }^{\circ} \mathrm{C}$ before use. Degassed and anhydrous tetrahydrofuran (THF), dichloromethane (DCM), benzene $\left(\mathrm{C}_{6} \mathrm{H}_{6}\right)$, and pentane were obtained from a JC Meyer solvent purification system. Toluene, 1,4-dioxane, and dimethylformamide (DMF) were dried over $3 \AA$ molecular sieves for at least 3 days before use. 1,2-dichloroethane (DCE) and chloroform $\left(\mathrm{CHCl}_{3}\right)$ were fractionally distilled and stored over $3 \AA$ molecular sieves before use. Triethyl orthoformate was dried over sodium sulfate and distilled from potassium hydroxide, degassed, and stored over $3 \AA$ molecular sieves before use. All moisture and air-sensitive reactions were performed under inert atmosphere (nitrogen) using standard Schlenk technique or, when noted, in a Vacuum Atmosphere glovebox. SiliaFlash F60 (40-63 $\mu \mathrm{m}, 230-400$ mesh) silica gel was used for column chromatography. Automated flash chromatography was performed using a Yamazen Smart Flash AKROS system.

\section{Characterization}

${ }^{1} \mathrm{H}$ nuclear magnetic resonance $\left({ }^{1} \mathrm{H} \mathrm{NMR}\right),{ }^{13} \mathrm{C}$ nuclear magnetic resonance $\left({ }^{13} \mathrm{C} \mathrm{NMR}\right)$, and ${ }^{31} \mathrm{P}$ nuclear magnetic resonance $\left({ }^{31} \mathrm{P}\right.$ NMR) spectra were obtained on a Bruker AVANCE-300, Bruker AVANCE500 , or Bruker DRX-500 NMR spectrometer. ${ }^{1} \mathrm{H}$ NMR spectra were taken in chloroform- $d\left(\mathrm{CDCl}_{3}\right.$, referenced to TMS, $\delta 0.00 \mathrm{ppm}), 1$,2-dichloroethane- $d 4\left(\mathrm{C}_{2} \mathrm{D}_{4} \mathrm{Cl}_{2}\right.$, referenced to residual $\mathrm{C}_{2} \mathrm{H}_{4} \mathrm{Cl}_{2}, \delta$ $3.72 \mathrm{ppm})$, and benzene- $d 6\left(\mathrm{C}_{6} \mathrm{D}_{6}\right.$, referenced to residual $\left.\mathrm{C}_{6} \mathrm{H}_{6}, \delta 7.16 \mathrm{ppm}\right)$. All ${ }^{13} \mathrm{C}$ NMR spectra were taken in chloroform- $d$ (referenced to chloroform, $\delta 77.16 \mathrm{ppm}$ ) and benzene- $d 6$ (referenced to benzene, $\delta 128.06 \mathrm{ppm}) .{ }^{31} \mathrm{P}$ NMR spectra were externally referenced to $85 \% \mathrm{H}_{3} \mathrm{PO}_{4}(0.00 \mathrm{ppm})$. Spectra were analyzed on TopSpin software. Chemical shifts are represented in parts per million (ppm); splitting patterns are assigned as s (singlet), d (doublet), t (triplet), q (quartet), p (quintet), m (multiplet), and br (broad); coupling constants, $J$, are reported in hertz (Hz).

Gel permeation chromatography (GPC) data were collected on Agilent 1260 HPLC equipped with a Wyatt 8-angle DAWN NEON light-scattering detector, ViscoStar NEON viscometer, and Optilab NEON refractive index detector. GPC samples were analyzed at a flow rate of $1 \mathrm{~mL} / \mathrm{min}$ in chloroform stabilized with 0.5\%-1.0\% ethanol through two Agilent PLgel MIXED-C columns at 35 ${ }^{\circ} \mathrm{C}$. $\mathrm{dn} / \mathrm{dc}$ values $(0.0480$ for poly $(\mathrm{AcNb})$ and 0.0974 for poly $(\mathrm{BnNb})$ ) were determined by the $100 \%$ mass recovery method using Wyatt Astra 7.3 software. High-resolution mass spectroscopy (HRMS) data were collected on an LTQ Orbitrap (ThermoScientific) operating in positive mode electrospray ionization. Instrument resolution was set to 60000 and elemental composition was confirmed by electrospray ionization HRMS. X-ray crystallography data was collected at $-173{ }^{\circ} \mathrm{C}(100 \mathrm{~K})$ on a Nonius Kappa CCD FR590 single crystal X-ray diffractometer, Mo-radiation. The data was integrated and scaled using SAINT, SADABS within the APEX2 software package by Bruker. 


\section{Synthetic and Experimental Procedures}

\section{Synthesis of 4-((6-bromohexyl)oxy)benzaldehyde (1)}

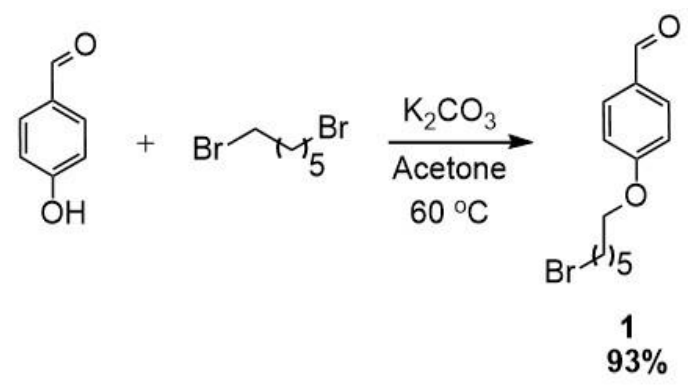

To a round bottom flask equipped with a stir bar was added 4-hydroxylaldehyde (5.00 g, $40.9 \mathrm{mmol})$, 1,6-dibromohexane $(18.9 \mathrm{~mL}, 123 \mathrm{mmol})$, potassium carbonate $(9.61 \mathrm{~g}, 69.6 \mathrm{mmol})$, and $90.0 \mathrm{~mL}$ (90 $\mathrm{mL})$. The round bottom flask was then attached to an air condenser and refluxed at $60{ }^{\circ} \mathrm{C}$ for $2 \mathrm{~d}$ (monitored by NMR). The reaction was cooled to room temperature and extracted with ethyl acetate $(100 \mathrm{~mL})$ and washed with water $(30 \mathrm{~mL} \times 3)$, brine $(20 \mathrm{~mL} \times 2)$. The organic layer was then dried over sodium sulfate, filtered, and concentrated under vacuum. The crude yellow oil was eluted with a DCM/hexane (50:50) solvent mixture on a silica column to yield 1 as a white solid $(11.2 \mathrm{~g}, 93 \%) .{ }^{1} \mathrm{H}$ NMR (300 MHz, CDCl $): \delta(\mathrm{ppm}) 9.86(\mathrm{~s}, 1 \mathrm{H}), 7.81(\mathrm{~d}, J=8.3 \mathrm{~Hz}, 2 \mathrm{H}), 6.97(\mathrm{~d}, J=8.3 \mathrm{~Hz}, 2 \mathrm{H}), 4.03$ $(\mathrm{t}, J=6.7 \mathrm{~Hz}, 2 \mathrm{H}), 3.41(\mathrm{t}, J=6.7 \mathrm{~Hz}, 2 \mathrm{H}), 1.85$ (overlap, 4H), 1.51 (overlap, 4H); ${ }^{13} \mathrm{C} \mathrm{NMR}(75 \mathrm{MHz}$, $\left.\mathrm{CDCl}_{3}\right): \delta(\mathrm{ppm}) 190.63,164.14,131.93,129.93,114.76,68.1433 .5332 .5928 .8727 .8325 .19$.

\section{Synthesis of 4-((6-(4-bromo-3,5-dimethylphenoxy)hexyl)oxy)benzaldehyde (2)}

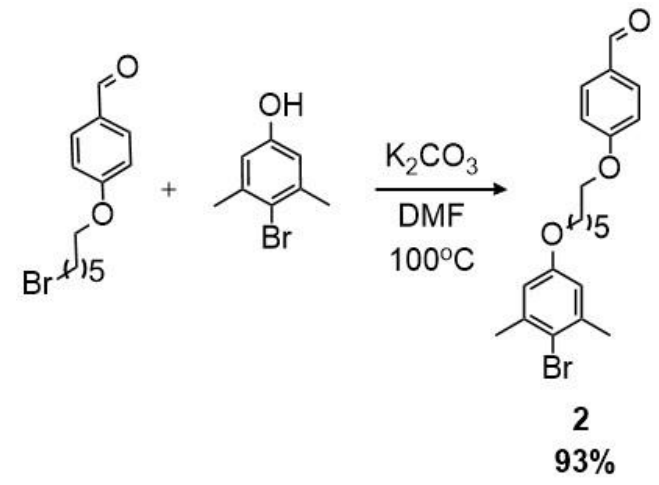

To a round bottom flask equipped with a stir bar was added 1 (4.00 g, $14.0 \mathrm{mmol}$ ), 4-bromo-3,5dimethylphenol (3.38 g, $16.8 \mathrm{mmol}$ ), and potassium carbonate $(3.29 \mathrm{~g}, 23.8 \mathrm{mmol})$ was added. The same flask was charged with DMF $(150 \mathrm{~mL})$ and the reaction mixture was stirred at $100{ }^{\circ} \mathrm{C}$ for $24 \mathrm{hr}$. The reaction was cooled to room temperature then extracted with ethyl acetate $(150 \mathrm{~mL})$. The organic layer was washed with $3 \%$ lithium chloride solution (15 mL x 3), DI water (15 mL x 5), and brine (15 $\mathrm{mL} \times 2$ ). The organic layer was dried over sodium sulfate, filtered, and concentrated under vacuum. The crude oil was purified on a silica column using ethyl acetate/hexane (2:8) solvent mixture to yield 2 as a white solid (5.68 g. 97\%). ${ }^{1} \mathrm{H}$ NMR $\left(300 \mathrm{MHz} \mathrm{CDCl}_{3}\right): \delta(\mathrm{ppm}) 9.86(\mathrm{~s}, 1 \mathrm{H}), 7.81(\mathrm{~d}, J=8.8 \mathrm{~Hz}$, $2 \mathrm{H}), 6.97(\mathrm{~d}, J=8.8 \mathrm{~Hz}, 2 \mathrm{H}), 6.63(\mathrm{~s}, 2 \mathrm{H}) 4.03(\mathrm{t}, J=6.5 \mathrm{~Hz}, 2 \mathrm{H}), 3.41(\mathrm{t}, J=6.5 \mathrm{~Hz}, 2 \mathrm{H}), 2.37$ (s, 
6H), 1.85 (overlap, 4H), 1.51 (overlap, 4H); ${ }^{13} \mathrm{C}$ NMR (75 MHz, $\mathrm{CDCl}_{3}$ ): $\delta(\mathrm{ppm})$ 190.64, 164.26, $157.77,139.03,131.93,129.93,114.76,114.47,68.23,67.85,29.15,28.99,25.75,23.96$. HRMS $\mathrm{m} /$ z calcd for $\mathrm{C}_{21} \mathrm{H}_{25} \mathrm{O}_{3}\left[\mathrm{M}^{+}\right]$405.106, found 405.106.

\section{Synthesis of 2-bromo-1,3-dimethyl-5-((6-(4-vinylphenoxy)hexyl)oxy)benzene (3)}

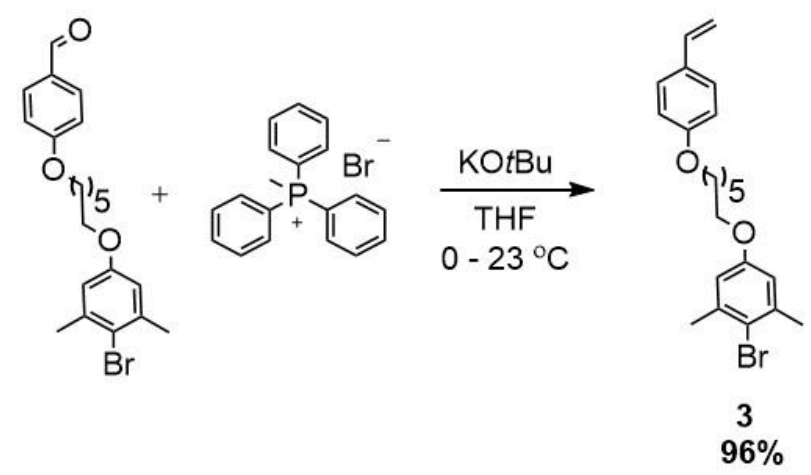

To a Schlenk flask equipped with a stir bar was added methyltriphenylphosphonium bromide (1.05 g, $2.96 \mathrm{mmol})$ and dry THF $(10 \mathrm{~mL})$. The flask was purged with nitrogen and the solution was cooled to $0{ }^{\circ} \mathrm{C}$. Potassium tert-butoxide $(0.550 \mathrm{~g}, 4.93 \mathrm{mmol})$ was then added to the Schlenk flask under a stream of nitrogen. The mixture was stirred at $0{ }^{\circ} \mathrm{C}$ for $10 \mathrm{~min}$. In a separate flask, $2(1.00 \mathrm{~g}, 246 \mathrm{mmol})$ was dissolved in $20 \mathrm{~mL}$ of dried THF under nitrogen and transferred (via cannula) to the Schlenk flask. The mixture was stirred at $0{ }^{\circ} \mathrm{C}$ for $15 \mathrm{~min}$ then the ice bath was removed. The reaction continued to stir at room temperature for $12 \mathrm{hr}$. Once the reaction was complete, the solution was quenched with methanol. Solvent was evaporated under vacuum. The residue was dissolved in DCM $(20 \mathrm{~mL})$ and washed with $\mathrm{H}_{2} \mathrm{O}(30 \mathrm{~mL} \times 3)$, and brine $(30 \mathrm{~mL} \times 3)$. The organic phase was then dried on sodium sulfate and concentrated under vacuum. The crude product was then loaded onto a silica column and the product was eluted with EtOAc/hexane (2:8) to yield 3 as a white solid $(0.920 \mathrm{~g}, 96 \%)$. ${ }^{1} \mathrm{H}$ NMR (300 MHz, $\left.\mathrm{CDCl}_{3}\right): \delta(\mathrm{ppm}) 7.35(\mathrm{~d}, J=8.8 \mathrm{~Hz}, 2 \mathrm{H}), 6.86(\mathrm{~d}, J=8.8 \mathrm{~Hz}, 2 \mathrm{H}), 6.69-6.57(\mathrm{~m}$, $1 \mathrm{H}) 6.63(\mathrm{~s}, 2 \mathrm{H}), 5.62(\mathrm{~d}, J=18.9 \mathrm{~Hz}, 1 \mathrm{H}), 5.13(\mathrm{~d}, J=11.9 \mathrm{~Hz}, 1 \mathrm{H}), 3.97-3.94$ (overlap, 4H), 2.39 (s, $6 \mathrm{H}), 1.84-1.81$ (overlap, 4H), 1.57-1.53 (overlap, 4H); ${ }^{13} \mathrm{C} \mathrm{NMR}\left(75 \mathrm{MHz}, \mathrm{CDCl}_{3}\right.$ ): $\delta(\mathrm{ppm}) 158.92$, $157.62,139.04,136.31,130.43,127.37,118.13,114.54,111.45,67.92,29.22,25.85,24.01$. HRMS m/z. calcd for $\mathrm{C}_{22} \mathrm{H}_{28} \mathrm{O}_{2}\left[\mathrm{M}^{+}\right]$403.1267, found 403.1267 . 


\section{Synthesis of $N$-mesitylethane-1,2-diamine-HBr salt (4a)}

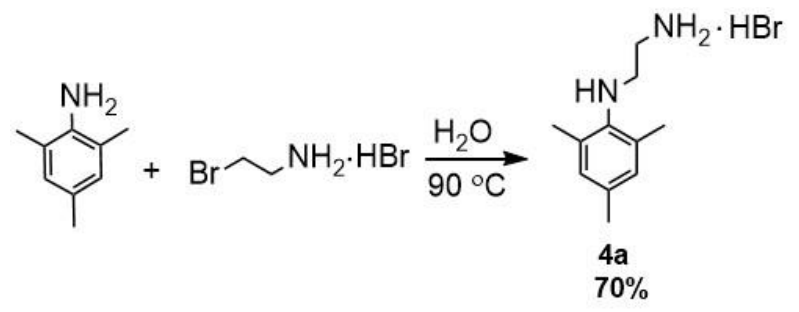

To a round bottom flask equipped with a stir bar was added 2,4,6-trimethyl aniline (20.0 g, $148 \mathrm{mmol})$ and 2-bromoethylamine hydrobromide $(15.2 \mathrm{~g}, 74.0 \mathrm{mmol})$. The solids were dissolved in $\mathrm{H}_{2} \mathrm{O}$ (40 $\mathrm{mL})$. The flask was equipped with a condenser and the mixture was stirred at $90{ }^{\circ} \mathrm{C}$ for $12 \mathrm{hr}$. The reaction was cooled to room temperature, extracted with water $(50 \mathrm{~mL})$, and washed with EtOAc $(50$ $\mathrm{mL} \times 3)$. The aqueous layer was collected and concentrated under vacuum. The solid residue was crystallized from hot EtOAc/methanol (2:1) to yield $\mathbf{4 a}$ as a white solid (13.4 g, 70\%) after filtering under vacuum and washing with minimal cold EtOAc. The solid $\mathrm{HBr}$ salt $4 \mathrm{a}$ could be stored for at least 3 months at a time in a dessicator on the benchtop. NMR data is consistent with reported values. ${ }^{1}$

\section{Synthesis of $N$-mesitylethane-1,2-diamine (4)}

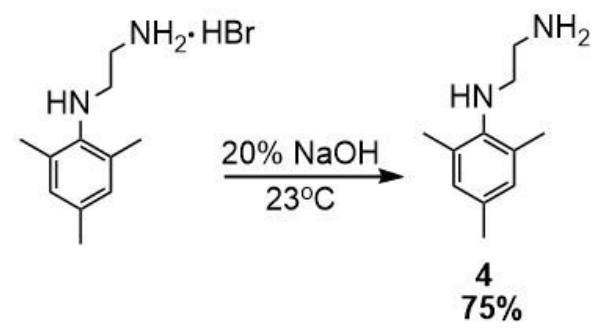

To a round bottom flask equipped with a stir bar was added $4 \mathrm{a}(1.00 \mathrm{~g}, 3.85 \mathrm{mmol})$ and 20\% aq. $\mathrm{NaOH}(20 \mathrm{~mL})$. The solution was stirred for $30 \mathrm{~min}$ at room temperature. The solution was extracted with DCM $(20 \mathrm{~mL}$ x 3$)$ and the combined organics were washed with water $(15 \mathrm{~mL}$ x 3$)$ and brine (15 $\mathrm{mL} \times 2$ ). The organic phase was dried over sodium sulfate and concentrated under vacuum to yield 4 as a yellow oil $(0.480 \mathrm{~g}, 70 \%)$. Fresh 4 was used within $24 \mathrm{hr}$ (stored under nitrogen at $4{ }^{\circ} \mathrm{C}$ ) after freebasing $\mathrm{HBr}$ salt $\mathbf{4 a}$. The yield of the subsequent amination reaction to generate $\mathbf{5}$ was significantly lower if 4 was not generated fresh. ${ }^{1} \mathrm{H}$ NMR $\left(300 \mathrm{MHz}, \mathrm{CDCl}_{3}\right): \delta(\mathrm{ppm}) 6.82(\mathrm{~s}, 2 \mathrm{H}), 2.97(\mathrm{~m}, 2 \mathrm{H})$ $2.89(\mathrm{~m}, 2 \mathrm{H}) 2.28(\mathrm{~s}, 6 \mathrm{H}), 2.22(\mathrm{~s}, 3 \mathrm{H}) ;{ }^{13} \mathrm{C} \mathrm{NMR}\left(75 \mathrm{MHz}, \mathrm{CDCl}_{3}\right): \delta(\mathrm{ppm}) 143.65,131.18,129.79$, 129.48, 51.34, 42.63, 20.60, 18.39 . 


\section{Synthesis of $\mathrm{N}$-(2,6-dimethyl-4-(4-(4-vinylphenoxy)butoxy)phenyl)-N-(3,4,5- trimethylphenyl)ethane-1,2-diamine (5)}

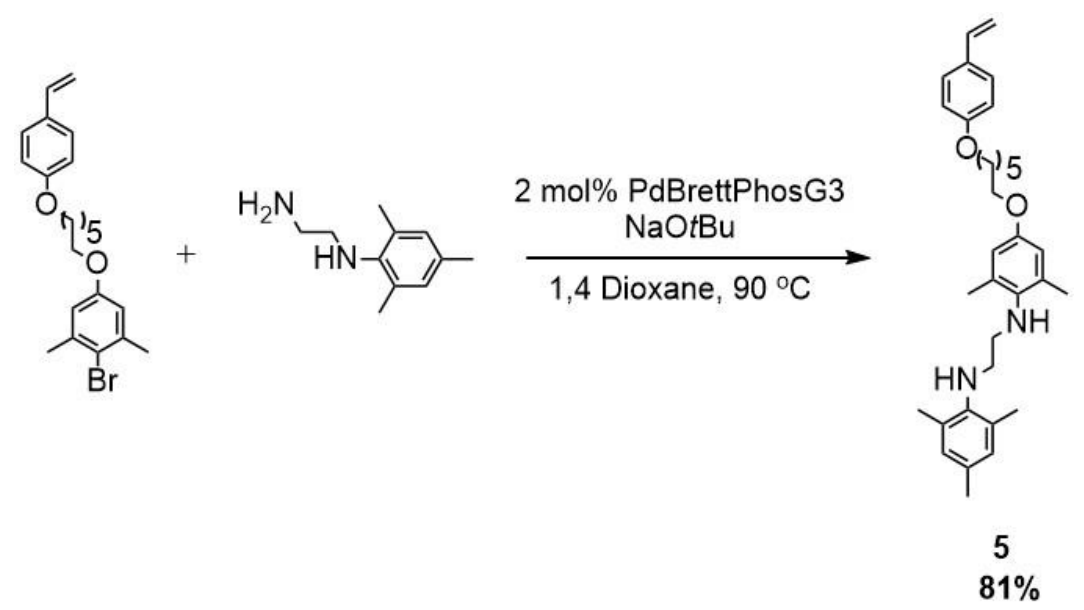

To a dry round bottom flask equipped with a stir bar was added $3(0.600 \mathrm{~g}, 1.48 \mathrm{mmol})$ and $4(0.420$ $\mathrm{g}, 2.38 \mathrm{mmol})$. The solids were dissolved in dry 1,4-dioxane and the solution was degassed by sparging with nitrogen for an hour. After an hour, the mixture was transferred (via cannula) to a separate Schlenk flask equipped with a stir bar containing sodium tert-butoxide $(0.230 \mathrm{~g}, 2.38 \mathrm{mmol})$ and PdBrettPhosG3 (0.0260 g, $0.0290 \mathrm{mmol})$ under nitrogen. The reaction mixture was stirred for $12 \mathrm{hr}$ at $90{ }^{\circ} \mathrm{C}$ (monitored by NMR). The reaction was cooled to room temperature and concentrated under vacuum. The crude mixture was loaded onto a silica column and the product was eluted with hexane/EtOAc/triethylamine (9:1:0.01). Product $\mathbf{5}$ was collected as a yellow oil $(0.610 \mathrm{~g}, 81 \%)$. ${ }^{1} \mathrm{H}$ $\operatorname{NMR}\left(300 \mathrm{MHz}, \mathrm{CDCl}_{3}\right): \delta(\mathrm{ppm}) 7.34(\mathrm{~d}, J=8.7 \mathrm{~Hz}, 2 \mathrm{H}), 6.89-6.84$ (overlap, $\left.4 \mathrm{H}\right), 6.67$ (dd, $J=17.6$, $10.9 \mathrm{~Hz}, 1 \mathrm{H}), 6.59(\mathrm{~s}, 2 \mathrm{H}), 5.61(\mathrm{~d}, J=17.6 \mathrm{~Hz}, 1 \mathrm{H}), 5.12(\mathrm{~d}, J=10.9 \mathrm{~Hz}, 1 \mathrm{H}), 3.97-3.91$ (overlap, 4H), 3.15-3.10 (overlap, 4H), 2.30 (s, 12H), 2.24 (s, 3H), 1.85-1.80 (overlap, 4H), 1.58-1.53 (overlap, $4 \mathrm{H}) ;{ }^{13} \mathrm{C}$ NMR $\left(75 \mathrm{MHz}, \mathrm{CDCl}_{3}\right): \delta(\mathrm{ppm}) 159.05,154.57,143.54,139.16,136.42,132.04,131.39$, $130.43,129.73,129.60,127.44,114.82,114.64,111.46,68.03,49.68,49.34,29.47,29.33,25.99,20.61$, 18.64, 18.50. HRMS $m / z$ calcd for $\mathrm{C}_{33} \mathrm{H}_{45} \mathrm{O}_{2} \mathrm{~N}_{2}\left[\mathrm{M}^{+}\right] 501.3476$, found 501.3481 . 
7. Synthesis of 3-(2,6-dimethyl-4-(4-(4-vinylphenoxy)butoxy)phenyl)-1-mesityl-4,5-dihydroimidazol-3-ium salt (6)

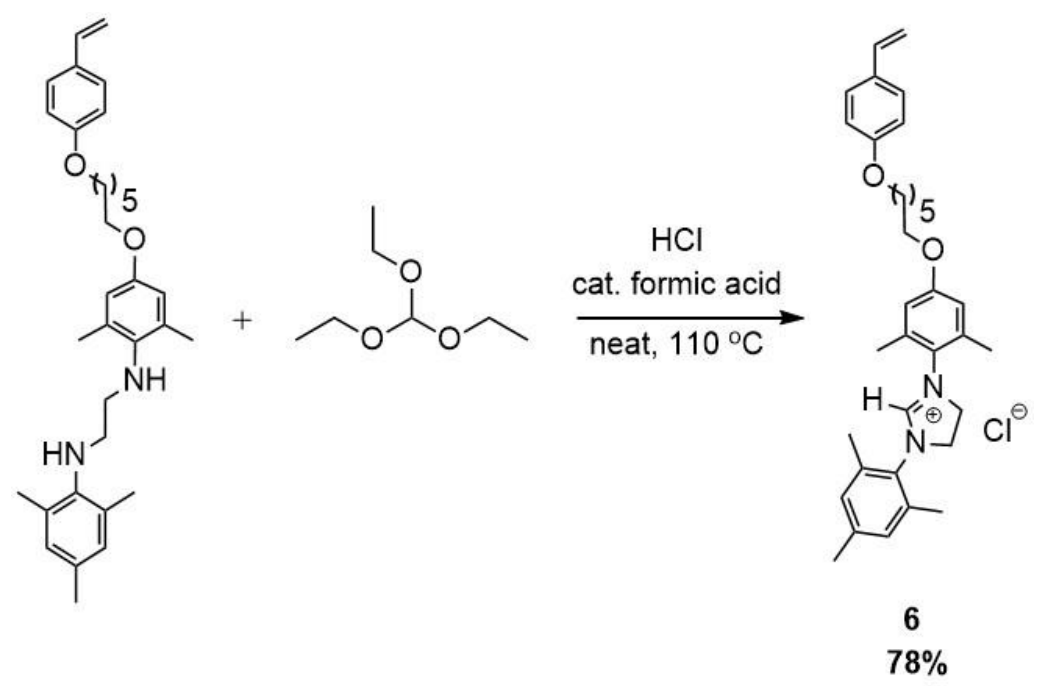

To a Schlenk flask equipped with a stir bar was added $5(0.400 \mathrm{~g}, 0.800 \mathrm{mmol})$ and dry toluene $(3 \mathrm{~mL})$ under nitrogen. $2 \mathrm{M} \mathrm{HCl}$ in diethyl ether $(0.660 \mathrm{~mL}, 6.07 \mathrm{mmol})$ was added to the flask and stirred for $20 \mathrm{~min}$ at room temperature. After 20 minutes, 1 drop of formic acid and triethyl orthoformate (4.03 $\mathrm{mL}, 24.4 \mathrm{mmol}$ ) were added to the vessel and the mixture was stirred at $110{ }^{\circ} \mathrm{C}$ for $15 \mathrm{~min}$. The reaction was cooled to room temperature and concentrated under vacuum. The crude mixture was loaded onto a silica column and the product was eluted with methanol/DCM (1:9). Product 6 was collected as a white solid $(0.260 \mathrm{~g}, 78 \%) .{ }^{1} \mathrm{H}$ NMR $\left(300 \mathrm{MHz}, \mathrm{CDCl}_{3}\right): \delta(\mathrm{ppm}) 9.27(\mathrm{~s}, 1 \mathrm{H}), 7.28(\mathrm{~d}, J$ $=8.7 \mathrm{~Hz}, 2 \mathrm{H}), 6.82(\mathrm{~s}, 2 \mathrm{H}), 6.81(\mathrm{~d}, J=8.7 \mathrm{~Hz}, 2 \mathrm{H}), 6.66-6.57$ (overlap, 3H), $5.56(\mathrm{~d}, J=18.6 \mathrm{~Hz}$, $1 \mathrm{H}), 5.08$ (d, $J=10.9 \mathrm{~Hz}, 1 \mathrm{H}), 4.52$ (s, 4H), 3.90-3.93 (overlap, 4H), 2.34 (s, 9H), 2.25 (s, 3H), 1.801.76 (overlap, 4H), 1.53-1.49 (overlap, 4H); ${ }^{13} \mathrm{C} \mathrm{NMR} \mathrm{(75} \mathrm{MHz,} \mathrm{CDCl}_{3}$ ): $\delta(\mathrm{ppm}) 160.42,159.57$, 158.81, 139.91, 136.69, 136.12, 134.93, 130.43, 130.21, 129.70, 127.20, 125.66, 114.71, 114.49, 111.31, 67.89, 67.82, 52.00, 51.82, 29.03, 28.95, 25.65, 20.84, 18.17, 17.78. HRMS $m /$ z calcd for $\mathrm{C}_{34} \mathrm{H}_{43} \mathrm{O}_{2} \mathrm{~N}_{2}$ $\left[\mathrm{M}^{+}\right]$511.3319, found 511.3319. 


\section{Synthesis of 1-(2,6-dimethyl-4-((6-(4-vinylphenoxy)hexyl)oxy)phenyl)-3-mesityl-2-}

(trichloromethyl)imidazolidine (7)
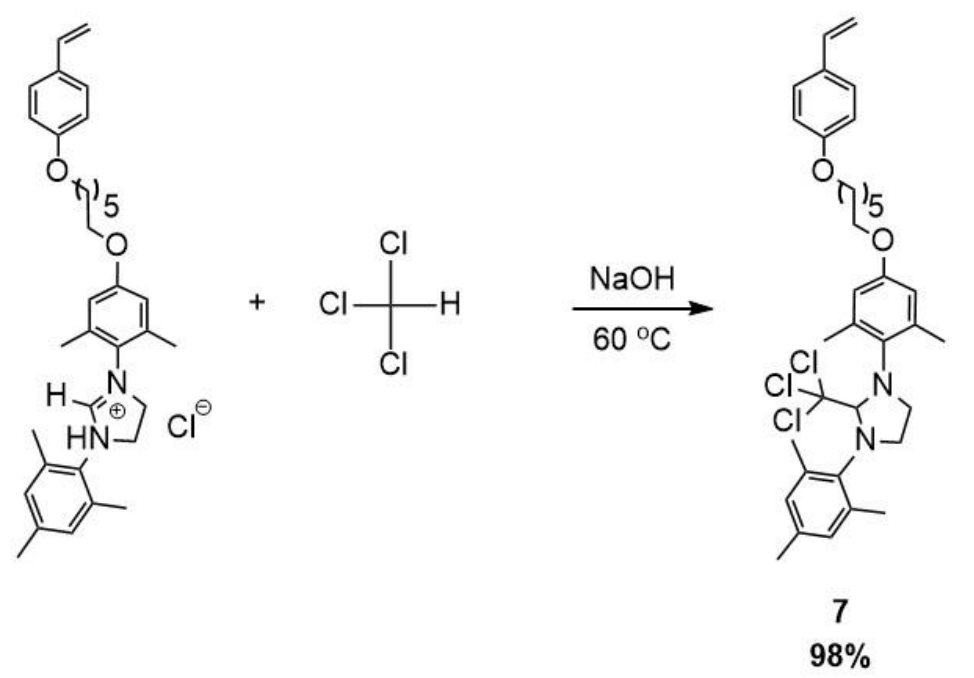

To a Schlenk flask was added freshly powdered (ground with mortar and pestel) sodium hydroxide $(0.100 \mathrm{~g}, 2.71 \mathrm{mmol})$, which was suspended in dry chloroform $(0.0730 \mathrm{~mL}, 0.910 \mathrm{mmol})$, followed by dry toluene $(8 \mathrm{~mL})$ under nitrogen. The mixture was stirred for $10 \mathrm{~min}$ and then $\mathbf{6}(0.100 \mathrm{~g}, 0.180$ mmol) was added to the flask under a stream of nitrogen. The flask was stirred at $60{ }^{\circ} \mathrm{C}$ for $2 \mathrm{hr}$. After the reaction was complete, the solution was cooled to room temperature and filtered through Celite. The filtrate was then concentrated under vacuum to yield 7 as a yellow oil $(0.110 \mathrm{~g}, 98 \%)$. 7 was used without further purification. ${ }^{1} \mathrm{H}$ NMR $\left(300 \mathrm{MHz}, \mathrm{CDCl}_{3}\right): \delta(\mathrm{ppm}) 7.22(\mathrm{~m}, 2 \mathrm{H}), 6.84-6.81$ (overlap, 4H), 6.68-6.55 (overlap, 3H), 5.58 (d, $J=17.6 \mathrm{~Hz}, 1 \mathrm{H}), 5.51$ (s, $1 \mathrm{H}), 5.09$ (d, $J=10.8 \mathrm{~Hz}, 1 \mathrm{H}), 3.94$ 3.92 (overlap, 6H), 3.28 (m, 2H), 2.46 (s, 9H), 2.33 (s, 3H), 2.23 (s, 3H), 1.81-1.77 (overlap, 4H), 1.531.48 (overlap, $4 \mathrm{H}) ;{ }^{13} \mathrm{C}$ NMR $\left(75 \mathrm{MHz}, \mathrm{CDCl}_{3}\right)$ : $\delta$ (ppm) 159.04, 156.41, 141.53, 140.17, 138.16, 137.13, 136.40, 135.58, 134.79, 133.89, 130.06, 129.83, 129.08, 128.27, 127.42, 125.36, 114.64, 111.45, 107.9, 86.42, 67.99, 67.77, 51.99, 51.76, 29.41, 29.30, 25.96, 21.45, 21.38, 20.68, 20.20, 19.92. 


\section{Synthesis of CB6}
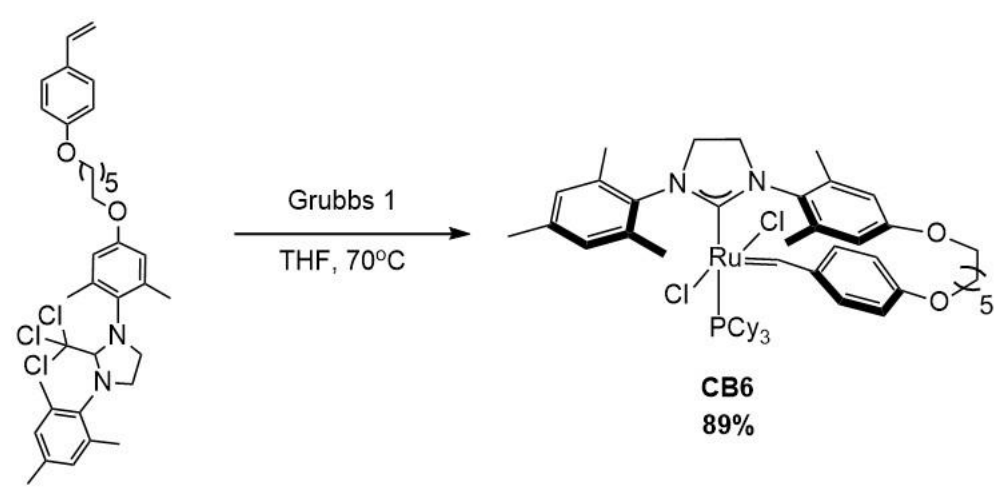

To a dry Schlenk flask equipped with a stir bar was added $7(0.100 \mathrm{~g}, 0.159 \mathrm{mmol})$, Grubbs $1^{\text {st }}$ generation catalyst $(0.0650 \mathrm{~g}, 0.0790 \mathrm{mmol})$, and dry THF $(80 \mathrm{~mL}, 2 \mathrm{mM})$ under nitrogen. The resulting solution was stirred at $70{ }^{\circ} \mathrm{C}$ for $1 \mathrm{hr}$ (monitored by NMR). After the reaction was complete, the mixture was cooled to room temperature then concentrated under vacuum (Figure S1a). The brown crude mixture was triturated in an ultrasonic bath with diethyl ether/pentane $(6: 4 ; 4 \mathrm{~mL}$ for every $100 \mathrm{mg}$ of crude mixture) (Figure S1b) until all solid was fully suspended. The suspension was then centrifuged at $10{ }^{\circ} \mathrm{C}(3000 \mathrm{rpm}, 10 \mathrm{~min})$ followed by decanting the supernatant (Figure S1c). This process was repeated 2 more times; after the $3^{\text {rd }}$ round, the decanted supernatant was colorless (Figure S1d). The collected microcrystalline tan solid was dried under vacuum (Figure S1e) to yield CB6 (0.066 g, 89\%). The following analytical data were collected after 3 rounds of washing, after which a single benzylidene peak is observed by ${ }^{1} \mathrm{H}$ NMR spectroscopy and a single phosphine peak is observed by ${ }^{31} \mathrm{P}$ NMR spectroscopy. Purity is $>20: 1$ based on ${ }^{1} \mathrm{H}$ NMR spectroscopy LOD. The crude ${ }^{1} \mathrm{H}$ and ${ }^{31} \mathrm{P}$ NMR spectra show the presence of other benzylidene-containing species, but we have been unable to elucidate their structure. Crystals for X-ray diffraction could be obtained by slow diffusion of pentane to a solution of $\mathbf{C B 6}$ in benzene (1:1 v/v). ${ }^{1} \mathrm{H}$ NMR $\left(500 \mathrm{MHz}, \mathrm{C}_{6} \mathrm{D}_{6}\right): \delta(\mathrm{ppm}) 19.40(\mathrm{~s}, 1 \mathrm{H}) 9.39$ (s, 1H), 7.32 (s, 1H), 6.98-6.93 (overlap, 2H), $6.92(\mathrm{~s}, 2 \mathrm{H}), 6.45(\mathrm{~m}, 2 \mathrm{H}), 5.91(\mathrm{~s}, 1 \mathrm{H}), 3.99(\mathrm{~s}, 1 \mathrm{H}), 3.65$ (s, 1H), 3.42-3.17 (overlap, 6H), 2.95 (s, 3H), $2.78(\mathrm{~s}, 3 \mathrm{H}), 2.62(\mathrm{~s}, 3 \mathrm{H}), 2.53(\mathrm{~m}, 3 \mathrm{H}), 2.42(\mathrm{~s}, 3 \mathrm{H}), 2.20$ $(\mathrm{s}, 3 \mathrm{H}), 1.77-1.11$ (overlap, 38H); ${ }^{13} \mathrm{C}$ NMR $\left(125 \mathrm{MHz}, \mathrm{C}_{6} \mathrm{D}_{6}\right): \delta(\mathrm{ppm}) 222.32\left(J_{\mathrm{CP}}=78.6 \mathrm{~Hz}\right), 158.79$, 158.51, 147.51, 139.43, 138.40, 138.23, 135.92, 135.37, 133.61, 132.71, 130.17, 117.06, 114.70, 113.68, 109.46, 66.17, 65.04, 52.17, 51.61, $32.16\left(\mathrm{~J}_{\mathrm{CP}}=16.0 \mathrm{~Hz}\right), 29.86,29.51,28.24\left(J_{\mathrm{CP}}=9.5 \mathrm{~Hz}\right), 27.92$, $27.17\left(J_{\mathrm{CP}}=11.3 \mathrm{~Hz}\right), 26.64,26.49,24.25,22.43,21.21,20.57,19.54 .{ }^{31} \mathrm{P} \mathrm{NMR}\left(121 \mathrm{MHz}, \mathrm{C}_{6} \mathrm{D}_{6}\right)$ : $\delta(\mathrm{ppm})$ 28.7. HRMS $m / z$ calcd for $\mathrm{C}_{51} \mathrm{H}_{73} \mathrm{O}_{2} \mathrm{~N}_{2} \mathrm{Cl}_{2} \mathrm{RuP}\left[\mathrm{M}^{+}\right]$948.3825, found 948.3782 .
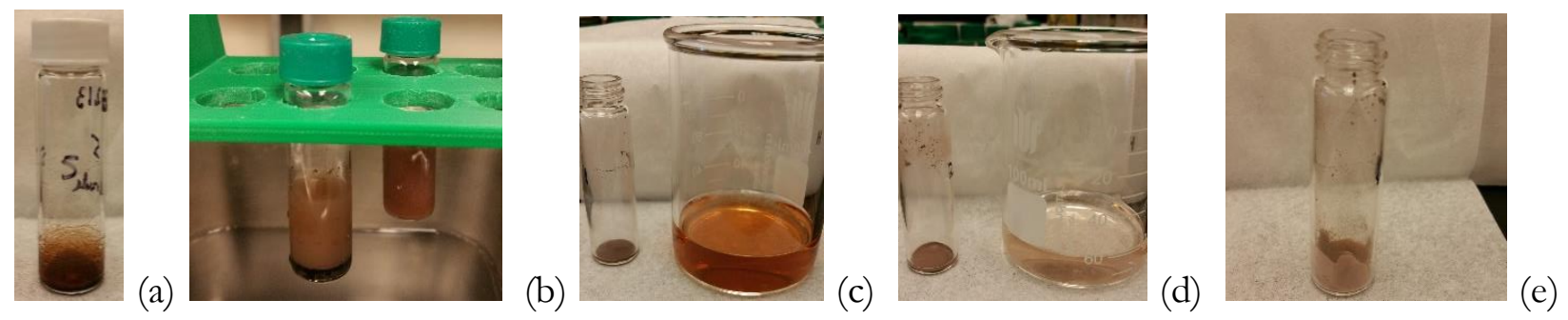

Figure S1. (a) Crude CB6; (b) Before (front) and after (back) $1^{\text {st }}$ round of sonication in $\mathrm{Et}_{2} \mathrm{O} / \mathrm{pentane}$; (c) Decanted supernatant after $1^{\text {st }}$ round of washing; (d) Decanted supernatant after $3^{\text {rd }}$ round of washing; (e) Dried solid CB6 


\section{Synthesis of $N$-mesitylimidazole (U1)}

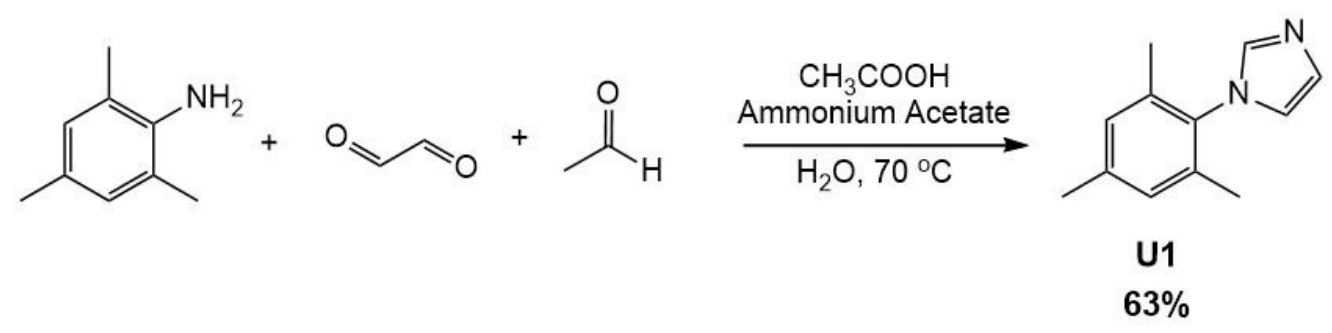

To a round bottom flask equipped a stir bar was added glacial acetic acid $(10.0 \mathrm{~mL}, 175 \mathrm{mmol})$, aqueous formaldehyde $(3.00 \mathrm{~mL}, 109 \mathrm{mmol})$ and aqueous glyoxal $(4.60 \mathrm{~mL}, 101 \mathrm{mmol})$. The resulting mixture was heated to $70{ }^{\circ} \mathrm{C}$. A freshly prepared solution of glacial acetic acid $(10.0 \mathrm{~mL}, 175 \mathrm{mmol})$, ammonium acetate $(3.08 \mathrm{~g}, 40.0 \mathrm{mmol})$ in water $(2 \mathrm{~mL})$, and 2,4,6-trimethylaniline $(5.60 \mathrm{~mL}, 39.9$ $\mathrm{mmol}$ ) were added dropwise to the reaction flask over a period of $35 \mathrm{~min}$. A condenser was attached to the round bottom flask and the solution was stirred for $18 \mathrm{hr}$ at $70^{\circ} \mathrm{C}$. The cooled reaction mixture was added dropwise to a stirring solution of $\mathrm{NaHCO}_{3}(29.4 \mathrm{~g})$ in water $(300 \mathrm{~mL})$ to precipitate brown solids. The precipitate was filtered, washed with water $(3 \times 20 \mathrm{~mL})$, and dried under vacuum. The dried solid was redissolved in minimal methanol, loaded onto a silica column, and eluted with methanol:DCM (1:19). Product U1 was collected as a brown solid (4.67 g, 63\%). 'NMR data is consistent with reported data. ${ }^{1} \mathrm{H}$ NMR $\left(500 \mathrm{MHz}, \mathrm{CDCl}_{3}\right): \delta(\mathrm{ppm}) 7.43(\mathrm{~m}, 1 \mathrm{H}), 7.23(\mathrm{~m}, 1 \mathrm{H}), 6.97$ (s, 2H), 6.89 (m, 1H), 2.34 (s, 3H), 1.99 (s, 6H).

\section{Synthesis of 3-(hex-5-en-1-yl)-1-mesityl-1-imidazol-3-ium salt (U2)}

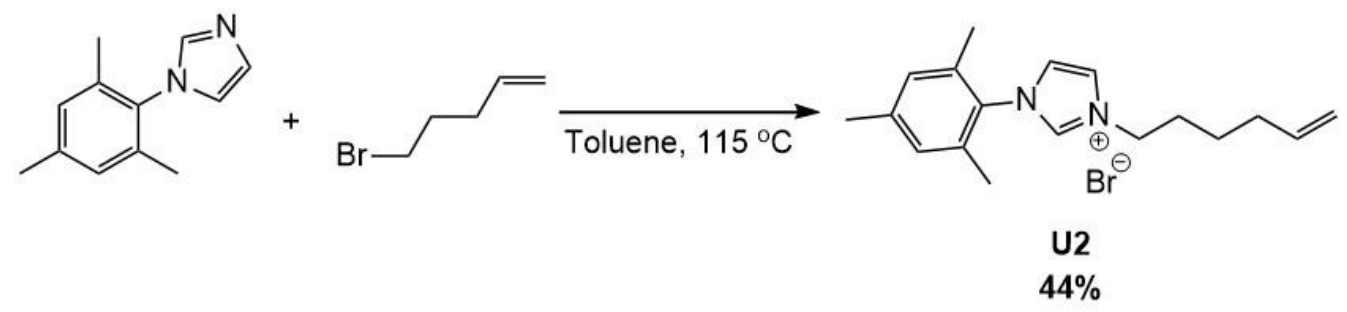

To a round bottom flask equipped with stir bar was added $\mathbf{U 1}$ (0.498 g, $2.67 \mathrm{mmol})$, 6-bromo-1-hexene $(0.523 \mathrm{~g}, 3.21 \mathrm{mmol})$, and toluene $(25 \mathrm{~mL})$. A condenser was attached to the round bottom flask and the solution was heated to $115^{\circ} \mathrm{C}$ under nitrogen for $21 \mathrm{hr}$. The crude reaction mixture was cooled to room temperature and concentrated under vacuum. The solid residue was redissolved in $1 \mathrm{~mL}$ of DCM and added slowly to flask containing $20 \mathrm{~mL}$ of Et $\mathrm{t}_{2} \mathrm{O}$. Immediate brown precipitates were formed. The precipitate was collected and dried under vacuum to yield U2 as a tan solid (0.410 g, 44\%). NMR data is consistent with reported data. ${ }^{3}{ }^{1} \mathrm{H}$ NMR $\left(500 \mathrm{MHz}, \mathrm{CDCl}_{3}\right): \delta(\mathrm{ppm}) 10.54(\mathrm{~s}, 1 \mathrm{H}), 7.68(\mathrm{br}$, 1H), $7.16(\mathrm{br}, 1 \mathrm{H}), 7.00(\mathrm{~s}, 2 \mathrm{H}), 5.74(\mathrm{ddt}, J=17.0,10.4,6.7 \mathrm{~Hz}, 1 \mathrm{H}), 5.01(\mathrm{~d}, J=17.0 \mathrm{~Hz}, 1 \mathrm{H}), 4.97$ $(\mathrm{d}, J=10.4 \mathrm{~Hz}, 1 \mathrm{H}), 4.77(\mathrm{~m}, 2 \mathrm{H}), 2.33(\mathrm{~s}, 3 \mathrm{H}), 2.13(\mathrm{~m}, 2 \mathrm{H}), 2.07(\mathrm{~s}, 6 \mathrm{H}), 2.01(\mathrm{~m}, 2 \mathrm{H}), 1.49(\mathrm{~m}, 2 \mathrm{H})$. 


\section{Synthesis of acyclic unsaturated 5-carbon ruthenium complex (UA5)}

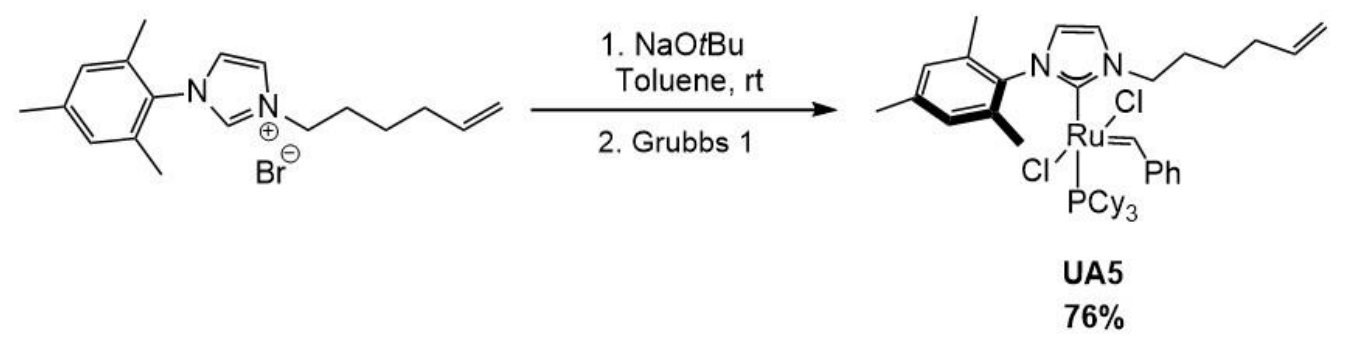

To a dry Schlenk flask equipped with a stir bar was added U2 $(0.205 \mathrm{~g}, 0.587 \mathrm{mmol})$, solid sodium tertbutoxide $(0.0564 \mathrm{~g}, 0.587 \mathrm{mmol})$, and dry toluene $(5 \mathrm{~mL})$. The reaction mixture was stirred under nitrogen for $2 \mathrm{hr}$ at room temperature. To the same flask, Grubbs $1^{\text {st }}$ generation catalyst $(0.241 \mathrm{~g}$, $0.293 \mathrm{mmol}$ ) was added under nitrogen and the reaction was stirred for an additional $1.5 \mathrm{hr}$. The crude solution was loaded onto deactivated silica (treated with $\mathrm{Et}_{3} \mathrm{~N}$ ) and the product was eluted with $\mathrm{Et}_{2} \mathrm{O}$. The collected fractions were dried under vacuum to yield a red/pink solid, UA5 (0.362 g, $76 \%)$. NMR data is consistent with reported data. ${ }^{3} \mathrm{H}$ NMR $\left(500 \mathrm{MHz}, \mathrm{CDCl}_{3}\right): \delta(\mathrm{ppm}) 19.26(\mathrm{~s}, 1 \mathrm{H}), 7.88(\mathrm{br}$, 2H), $7.40(\mathrm{t}, J=7.3 \mathrm{~Hz}, 1 \mathrm{H}), 7.20(\mathrm{~d}, J=1.8 \mathrm{~Hz}, 1 \mathrm{H}), 7.11(\mathrm{~m}, 2 \mathrm{H}), 6.80(\mathrm{~d}, J=1.8 \mathrm{~Hz}, 1 \mathrm{H}), 6.29$ (br, $2 \mathrm{H}), 5.86(\mathrm{ddt}, J=17.1,10.4,6.7 \mathrm{~Hz}, 1 \mathrm{H}), 5.10-4.70$ (overlap, $2 \mathrm{H}), 4.72(\mathrm{t}, J=7.7 \mathrm{~Hz}, 2 \mathrm{H}), 2.42-2.28$ (overlap, 4H), 2.26-2.15 (overlap, 4H), $2.00-1.84$ (overlap, 8H), $1.77-1.50$ (overlap, 14H), 1.44-1.23 (overlap, 8H), $1.23-1.03$ (overlap, 10H).

\section{Synthesis of unsaturated cyclic 5-carbon ruthenium complex (UC5)}
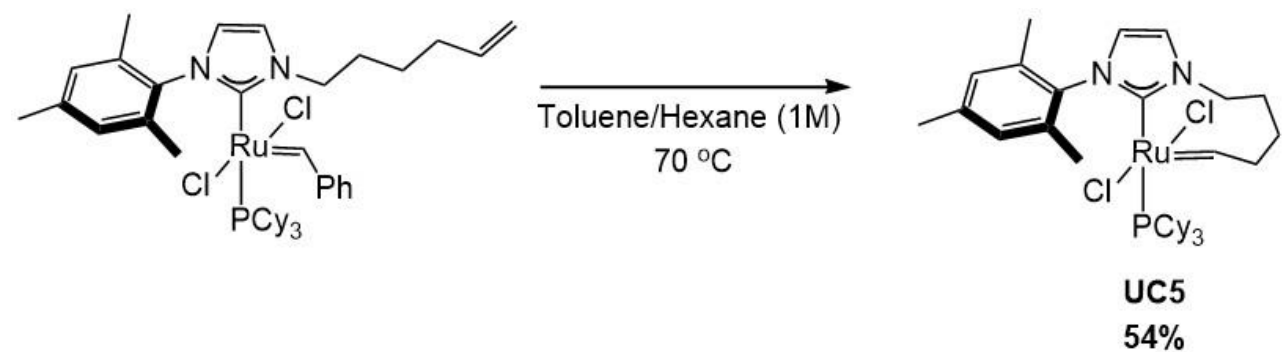

To a dry Schlenk flask equipped with a stir bar was added UA5 (0.170 g, $0.209 \mathrm{mmol})$, degassed toluene $(3 \mathrm{~mL})$, and degassed hexane $(200 \mathrm{~mL})$. The solution was stirred at $70{ }^{\circ} \mathrm{C}$ for $1 \mathrm{hr}$ then cooled to room temperature. The mixture was concentrated under vacuum. The crude residue was triturated with diethyl ether/pentane (1:4; $4 \mathrm{~mL}$ for every $100 \mathrm{mg}$ of crude mixture), filtered, and the collected solid was dried under vacuum. The process was repeated 3 times to yield UC5 as a green solid (0.0650 g, 54\%). NMR data is consistent with reported data. ${ }^{3} \mathrm{H}$ NMR $\left(500 \mathrm{MHz}, \mathrm{CDCl}_{3}\right): \delta(\mathrm{ppm}) 20.25(\mathrm{dt}$, $J=10.0,5.2 \mathrm{~Hz}, 1 \mathrm{H}), 7.03(\mathrm{~d}, J=2.1 \mathrm{~Hz}, 1 \mathrm{H}), 6.99(\mathrm{~s}, 2 \mathrm{H}), 6.81(\mathrm{~d}, J=2.1 \mathrm{~Hz}, 1 \mathrm{H}), 3.58(\mathrm{~m}, 2 \mathrm{H})$, $3.00(\mathrm{~m}, 2 \mathrm{H}), 2.41-2.15$ (overlap, 14H), $1.84-1.59$ (overlap, 17H), $1.35-1.06$ (overlap, 15H). 


\section{Synthesis of $\mathbf{N}$-benzyl-exo-norbornene-2,3-dicarboximide monomer $(\mathrm{BnNb})$}

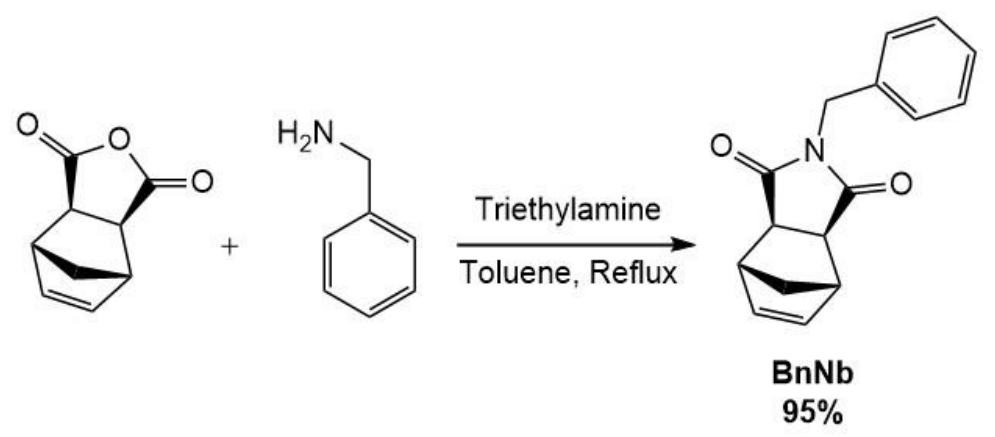

To a round bottom flask equipped with a stir bar was added cis-5-norbornene-exo-2,3-dicarboxylic anhydride $(1.00 \mathrm{~g}, 6.09 \mathrm{mmol})$, benzylamine $(0.730 \mathrm{~mL}, 6.70 \mathrm{mmol})$, triethylamine $(0.930 \mathrm{~mL}, 6.70$ mmol), and dry toluene $(35 \mathrm{~mL})$. The reaction mixture was stirred at reflux for $20 \mathrm{hr}$ under nitrogen. The reaction mixture was cooled to temperature and extracted with EtOAc $(30 \mathrm{~mL})$ and was washed with $10 \% \mathrm{HCl}(2 \times 15 \mathrm{~mL})$, water $(3 \times 15 \mathrm{~mL})$, and brine $(2 \times 15 \mathrm{~mL})$. The organic phase was collected and dried with sodium sulfate. The filtered solution was concentrated under vacuum to yield a yellow solid. The solid was further purified by crystallization in DCM to obtain $\mathbf{B n N b}$ as a white solid (1.47 g, 95\%). ${ }^{1} \mathrm{H}$ NMR $\left(300 \mathrm{MHz}, \mathrm{CDCl}_{3}\right.$ ): $\delta(\mathrm{ppm})$ 7.44-7.31 (overlap, $\left.5 \mathrm{H}\right), 6.33(\mathrm{~s}, 2 \mathrm{H}), 4.68(\mathrm{~s}, 2 \mathrm{H}), 3.31$ $(\mathrm{s}, 2 \mathrm{H}), 2.74(\mathrm{~s}, 2 \mathrm{H}), 1.47(\mathrm{~d}, J=9.9 \mathrm{~Hz}, 1 \mathrm{H}), 1.12(\mathrm{~d}, J=9.9 \mathrm{~Hz}, 1 \mathrm{H}) ;{ }^{13} \mathrm{C} \mathrm{NMR}\left(75 \mathrm{MHz}, \mathrm{CDCl}_{3}\right)$ : $\delta(\mathrm{ppm}) 177.66,137.95,135.94,128.89,128.65,127.92,47.83,45.33,42.65,42.38$.

\section{Synthesis of $\mathbf{N}$-ethyl hydroxyl-exo-norbornene-2,3-dicarboximide (8)}
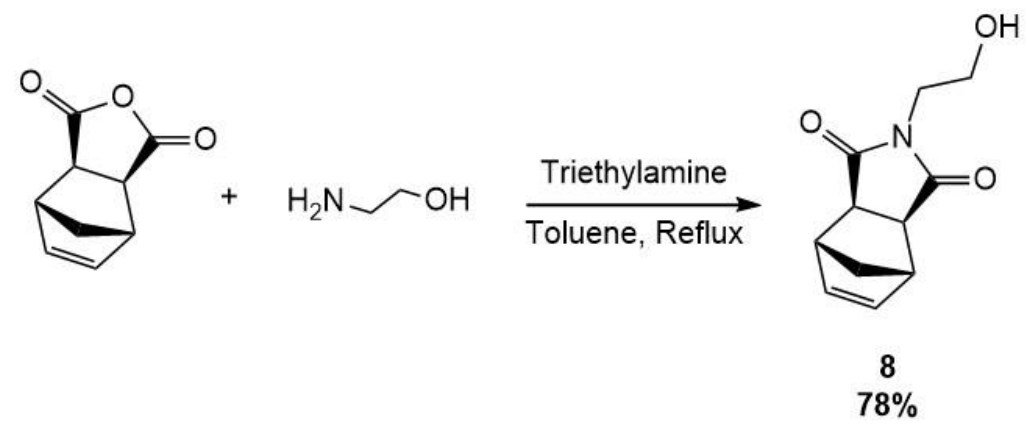

To a round bottom flask equipped with a stir bar was added cis-5-norbornene-exo-2,3-dicarboxylic anhydride $(1.00 \mathrm{~g}, 6.09 \mathrm{mmol})$, ethanolamine $(0.460 \mathrm{~mL}, 7.61 \mathrm{mmol})$, triethylamine $(0.110 \mathrm{~mL}, 7.61$ $\mathrm{mmol})$, and dry toluene $(35 \mathrm{~mL})$. The reaction mixture was stirred at reflux for $12 \mathrm{hr}$ under nitrogen. The reaction mixture was cooled to room temperature, extracted with EtOAc $(30 \mathrm{~mL})$, and washed with $10 \% \mathrm{HCl}(15 \mathrm{~mL} \times 3)$, water $(15 \mathrm{~mL} \times 3)$ and brine $(15 \mathrm{~mL}$ x 3). The organic phase was collected and dried with sodium sulfate. The filtered solution was concentrated under vacuum to yield $\mathbf{8}$ as a white solid (1.18 g, 78\%). ${ }^{1} \mathrm{H}$ NMR $\left(300 \mathrm{MHz} \mathrm{CDCl}_{3}\right): \delta(\mathrm{ppm}) 6.29(\mathrm{~s}, 2 \mathrm{H}), 3.78(\mathrm{t}, J=5.2 \mathrm{~Hz}, 2 \mathrm{H})$, $3.70(\mathrm{t}, J=5.2,2 \mathrm{H}), 3.29(\mathrm{~s}, 2 \mathrm{H}), 2.72(\mathrm{~s}, 2 \mathrm{H}), 2.13(\mathrm{br}, 1 \mathrm{H}), 1.52(\mathrm{~d}, J=9.9 \mathrm{~Hz}, 1 \mathrm{H}), 1.35(\mathrm{~d}, J=9.9$ $\mathrm{Hz}, 1 \mathrm{H}) ;{ }^{13} \mathrm{C} \mathrm{NMR}\left(75 \mathrm{MHz}, \mathrm{CDCl}_{3}\right): \delta(\mathrm{ppm}) 178.71,137.84,60.35,47.91,45.29,42.79,41.33$. 


\section{Synthesis of $\mathrm{N}$-ethyl acetoxy-exo-norbornene-2,3-dicarboximide (AcNb)}

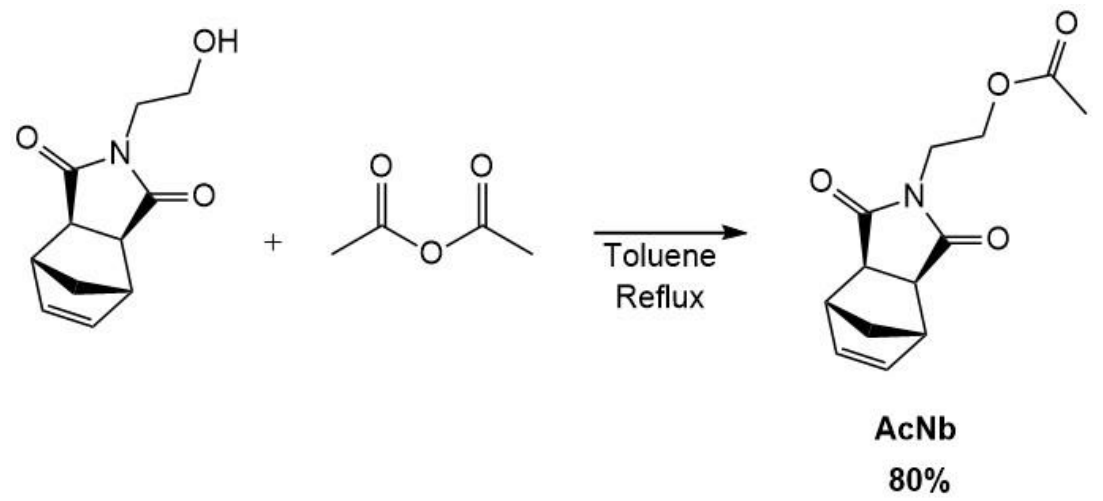

To a round bottom flask equipped with a stir bar was added $8(1.00 \mathrm{~g}, 4.82 \mathrm{mmol})$ and toluene (30 $\mathrm{mL})$. Once dissolved, acetic anhydride $(1.37 \mathrm{~mL}, 14.5 \mathrm{mmol})$ was added to the flask. The mixture was stirred at reflux for $12 \mathrm{hr}$. After the reaction was complete, the mixture was cooled to $0{ }^{\circ} \mathrm{C}$ and $40 \mathrm{aq}$ $\mathrm{wt} \%$ methylamine was added dropwise in excess $(1 \mathrm{~mL})$ to quench the reaction. The resulting crude mixture was extracted with EtOAc and washed with $10 \%$ aq. $\mathrm{NaHCO}_{3}(15 \mathrm{~mL}$ x 2), water $(15 \mathrm{~mL} x$ $3)$, and brine $(15 \mathrm{~mL} \times 2)$. The organic phase was collected and dried over sodium sulfate. The solution was filtered and concentrated under vacuum. The crude oil was loaded onto a silica column and purified with hexane/EtOAc (2:1) to yield $\mathbf{A c N b}$ as a yellow oil (0.962 g, 80\%). ${ }^{1} \mathrm{H}$ NMR (300 MHz, $\left.\mathrm{CDCl}_{3}\right): \delta(\mathrm{ppm}) 6.29(\mathrm{~s}, 2 \mathrm{H}), 4.22(\mathrm{t}, J=5.2 \mathrm{~Hz}, 2 \mathrm{H}), 3.75(\mathrm{t}, J=5.2 \mathrm{~Hz}, 2 \mathrm{H}), 3.28(\mathrm{~s}, 2 \mathrm{H}), 2.70$ (s, $2 \mathrm{H}), 1.99(\mathrm{~s}, 3 \mathrm{H}), 1.51(\mathrm{~d}, J=9.8 \mathrm{~Hz}, 1 \mathrm{H}), 1.30(\mathrm{~d}, J=9.8 \mathrm{~Hz}, 1 \mathrm{H}) ;{ }^{13} \mathrm{C}$ NMR $\left(75 \mathrm{MHz}, \mathrm{CDCl}_{3}\right)$ : $\delta(\mathrm{ppm}) 177.81,170.61,137.82,60.66,47.84,45.27,42.64,37.55,20.68$.

\section{Representative general procedure for REMP reaction with CB6}

In a nitrogen-filled glovebox, a $2 \mathrm{~mL}$ (8-425) screw-cap vial was charged with monomer $\mathbf{A c N b}(0.0200$ g, $0.0800 \mathrm{mmol})$ and DCE $(0.363 \mathrm{~mL})$. The solution was heated to $55^{\circ} \mathrm{C}$, then an aliquot of freshly prepared CB6 stock solution $(0.038 \mathrm{~mL}, 0.01 \mathrm{M} \mathrm{CB6}$ in DCE) was added via micropipette to afford a final monomer concentration of $0.200 \mathrm{M}$. The vial was sealed with a solid PTFE-lined cap and the polymerization reaction was stirred at $55^{\circ} \mathrm{C}$. The reaction vial was cooled to room temperature at the desired reaction time. The polymer solution was added dropwise into a stirring cold methanol solution. The pale yellow/white precipitate was collected using a centrifuge and dried under vacuum. The solid was redissolved in DCM and reprecipitated in cold methanol two additional times to afford a white solid polymer. The resulting white solid was dried under vacuum, dissolved in chloroform, filtered through a $0.2 \mu \mathrm{m}$ syringe filter, and analyzed by GPC-MALS-IV. Identical procedures were used to prepare polymers from $\boldsymbol{U} \boldsymbol{C} 5\left(55^{\circ} \mathrm{C}\right.$ in DCE) and $\boldsymbol{G} \mathbf{2}\left(40^{\circ} \mathrm{C}\right.$ in DCM). 


\section{General procedure for ${ }^{1} \mathrm{H}$ NMR spectroscopy reaction kinetics experiments}

The target monomer and initiator were brought into a nitrogen-filled glove box. In a dry "threadedtop" NMR tube, the monomer $(0.000580 \mathrm{mmol})$ was dissolved in DCE- $d 4\left(\mathrm{C}_{2} \mathrm{D}_{4} \mathrm{Cl}_{2}, 0.450 \mathrm{~mL}\right)$ and the NMR tube was sealed with a septum screw cap (8-425 thread size). In a separate vial, the desired Ru initiator, CB6, UC5, or G2, $(0.0290 \mathrm{mmol})$ was dissolved in $\mathrm{C}_{2} \mathrm{D}_{4} \mathrm{Cl}_{2}(0.0500 \mathrm{~mL})$ and was fitted with a septum screw cap. The contents of the NMR tube were thermally equilibrated in the NMR probe at $55^{\circ} \mathrm{C}$ for $5 \mathrm{~min}$. The tube was removed from the spectrometer and the previously prepared Ru-initiator solution was immediately injected into the NMR tube via microsyringe. The NMR tube was quickly inverted to mix the contents and then was immediately reinserted into the spectrometer for data collection. The time between injection of the $\mathrm{Ru}$ initiator and the collection of the first spectrum was at least 5 seconds. Reactions were monitored by comparing the integration of the diagnostic cis-alkene monomer peak (ca. $5.8-6.2 \mathrm{ppm}$ ) at a given time point to the integration of the same diagnostic peak at $t=0 \mathrm{~s}$. This "initial time point" sample is a solution of target monomer at the initial working concentration $(0.000580 \mathrm{mmol} / 0.500 \mathrm{~mL}=1.16 \mathrm{mM})$; it is indicative of a sample with the expected diagnostic monomer peak integration after dilution with the initiator solution (assuming no monomer reacts initially). All integrations were normalized against residual 1,2-dichloroethane $(\delta$ 3.7 ppm). See Tables S7 - S9 (pp. S58 - S61) for the raw data used to generate the plot in Figure 4a in the main text. Please note that a short d1 value $(0.1 \mathrm{~s})$ had to be used for $\mathbf{G} \mathbf{2}$ data acquisition in order to record a sufficient number of time points under these experimental conditions. 


\section{NMR Spectroscopy}
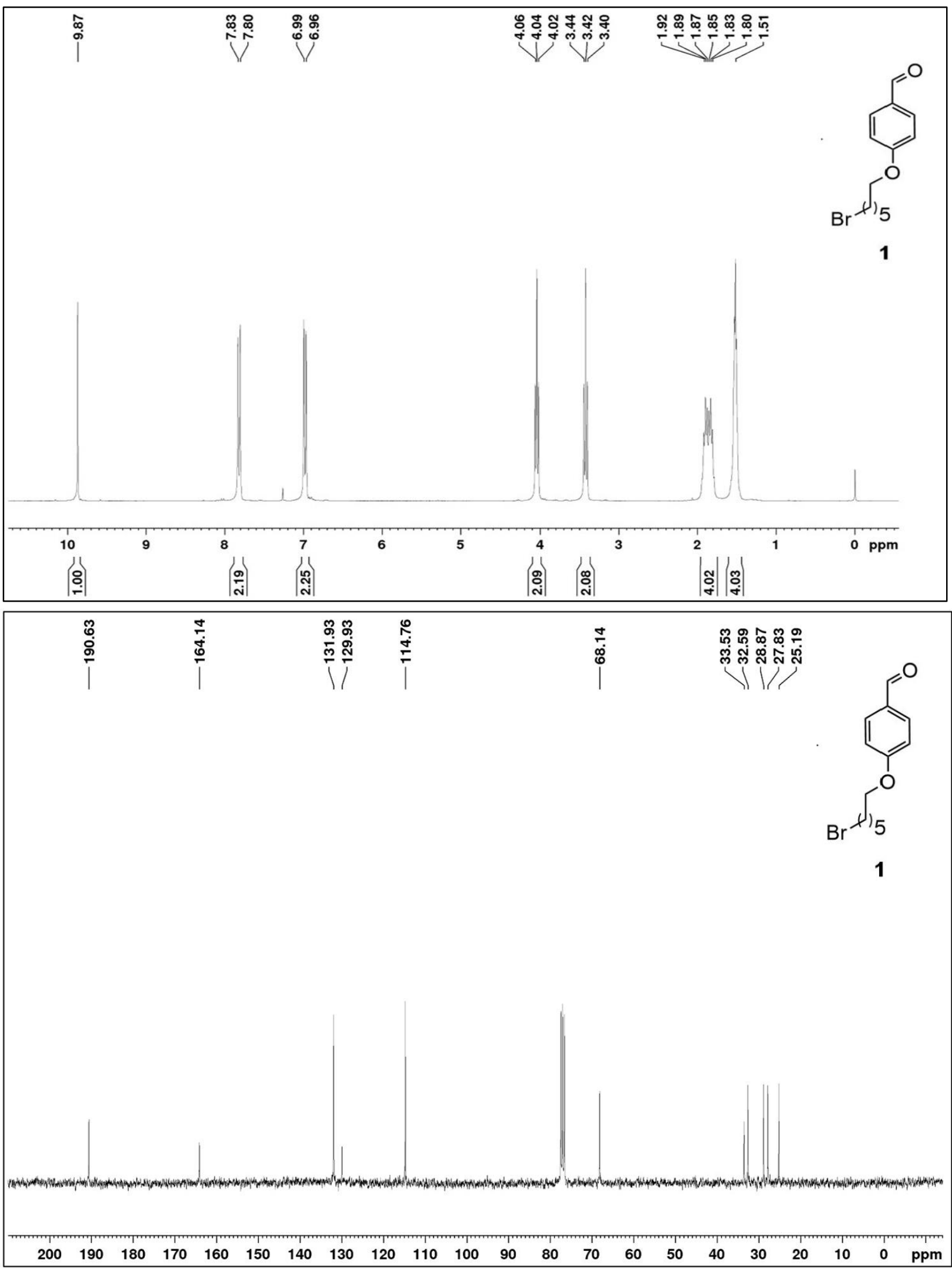

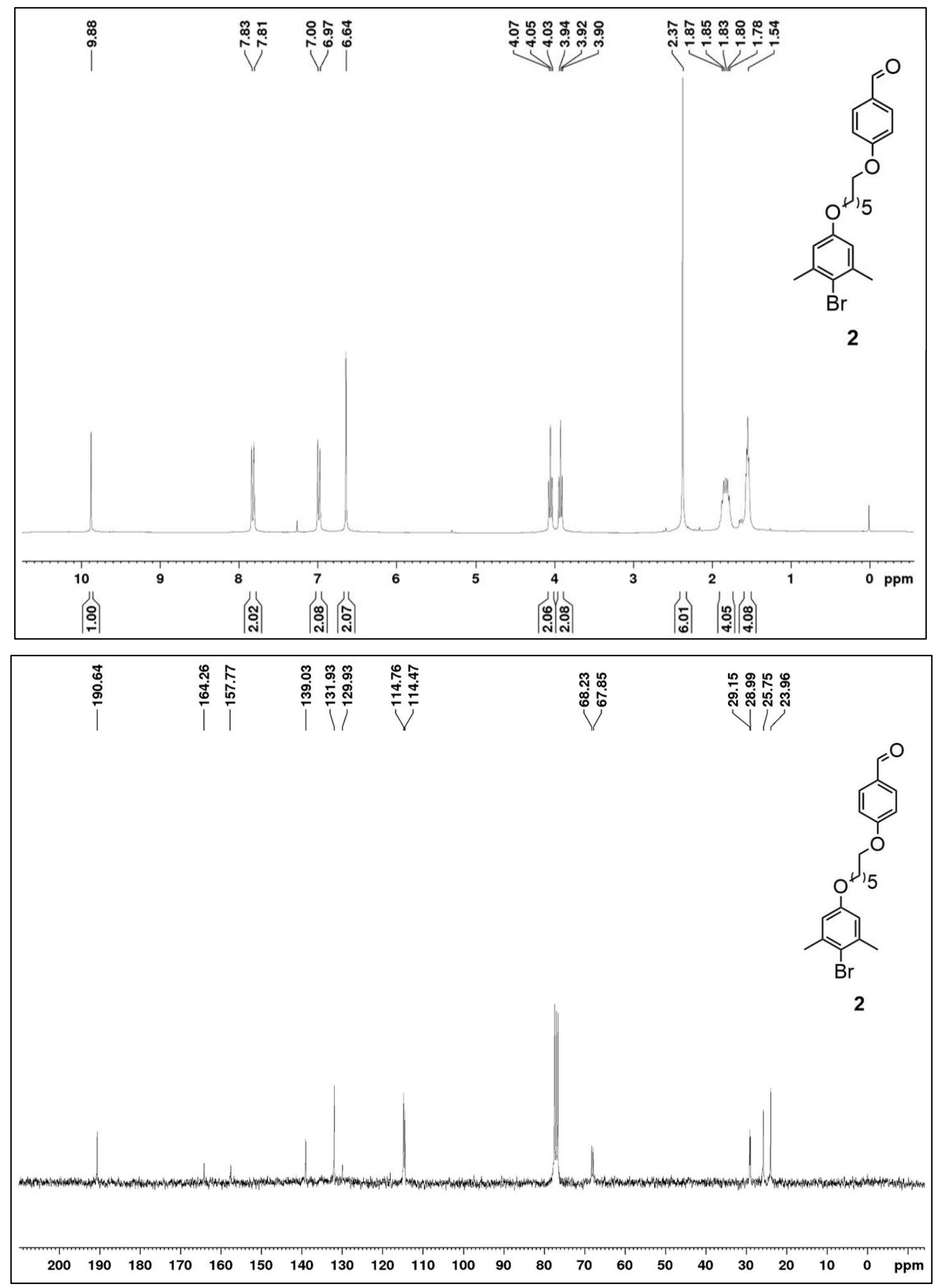

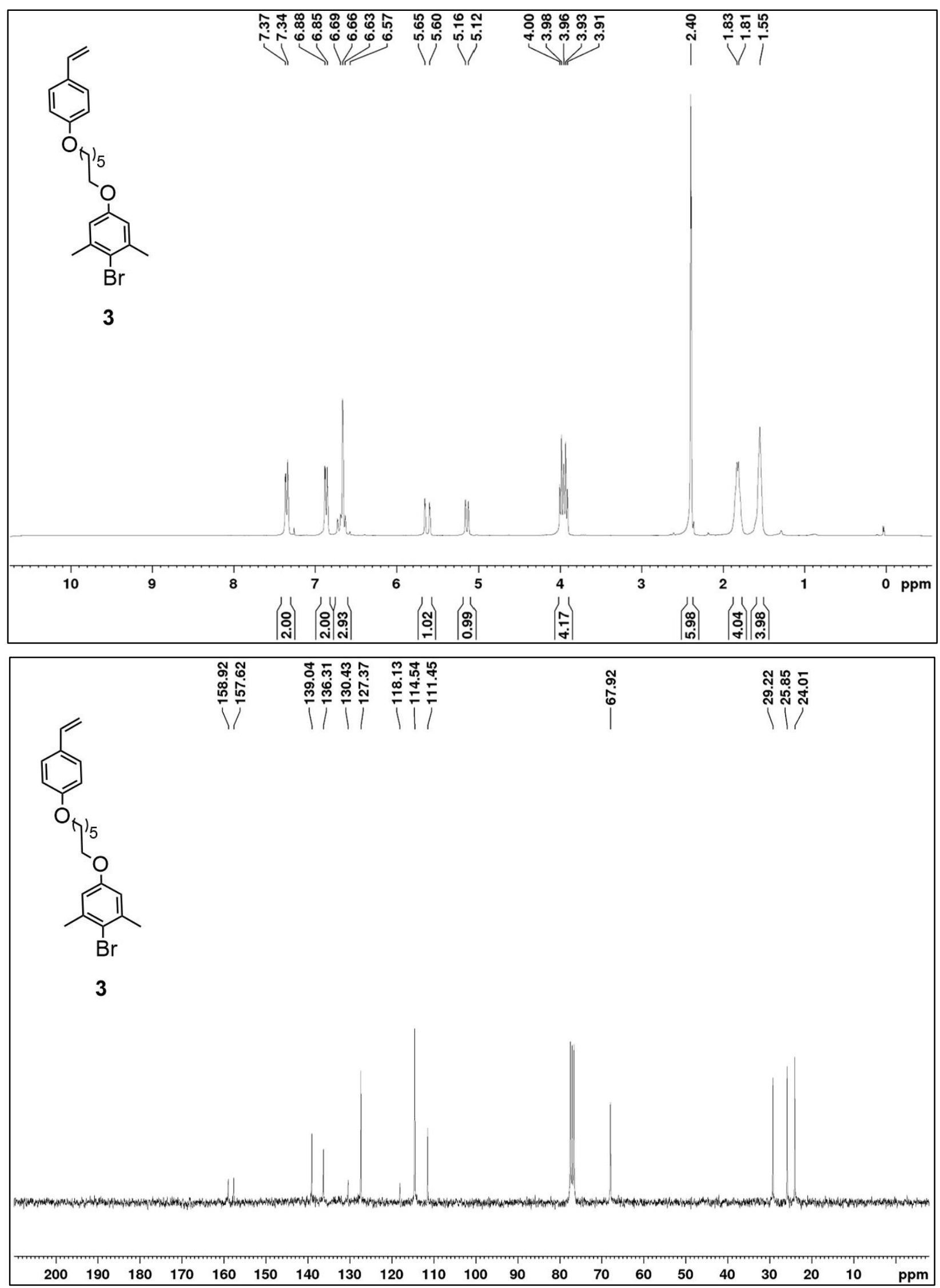

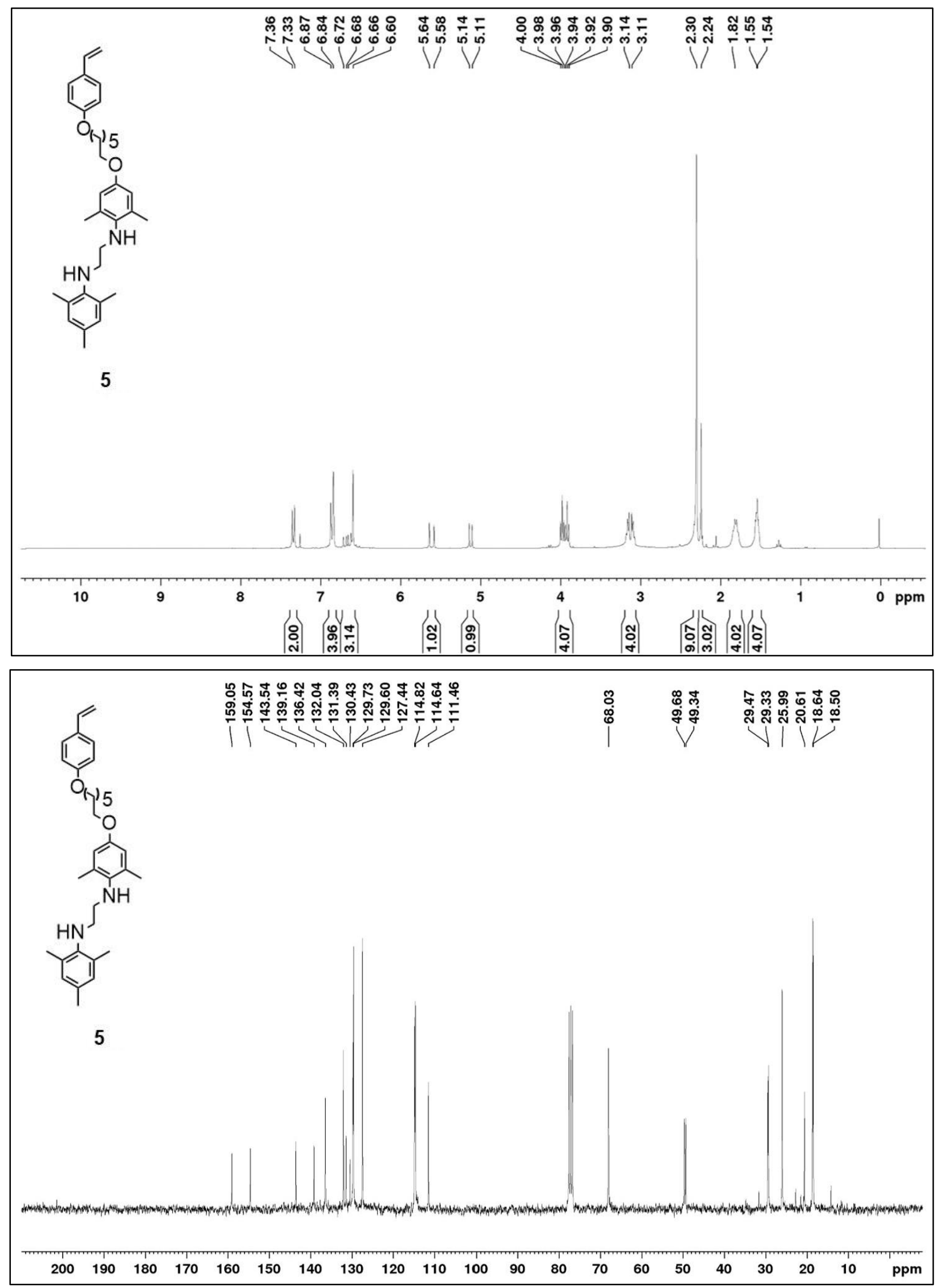

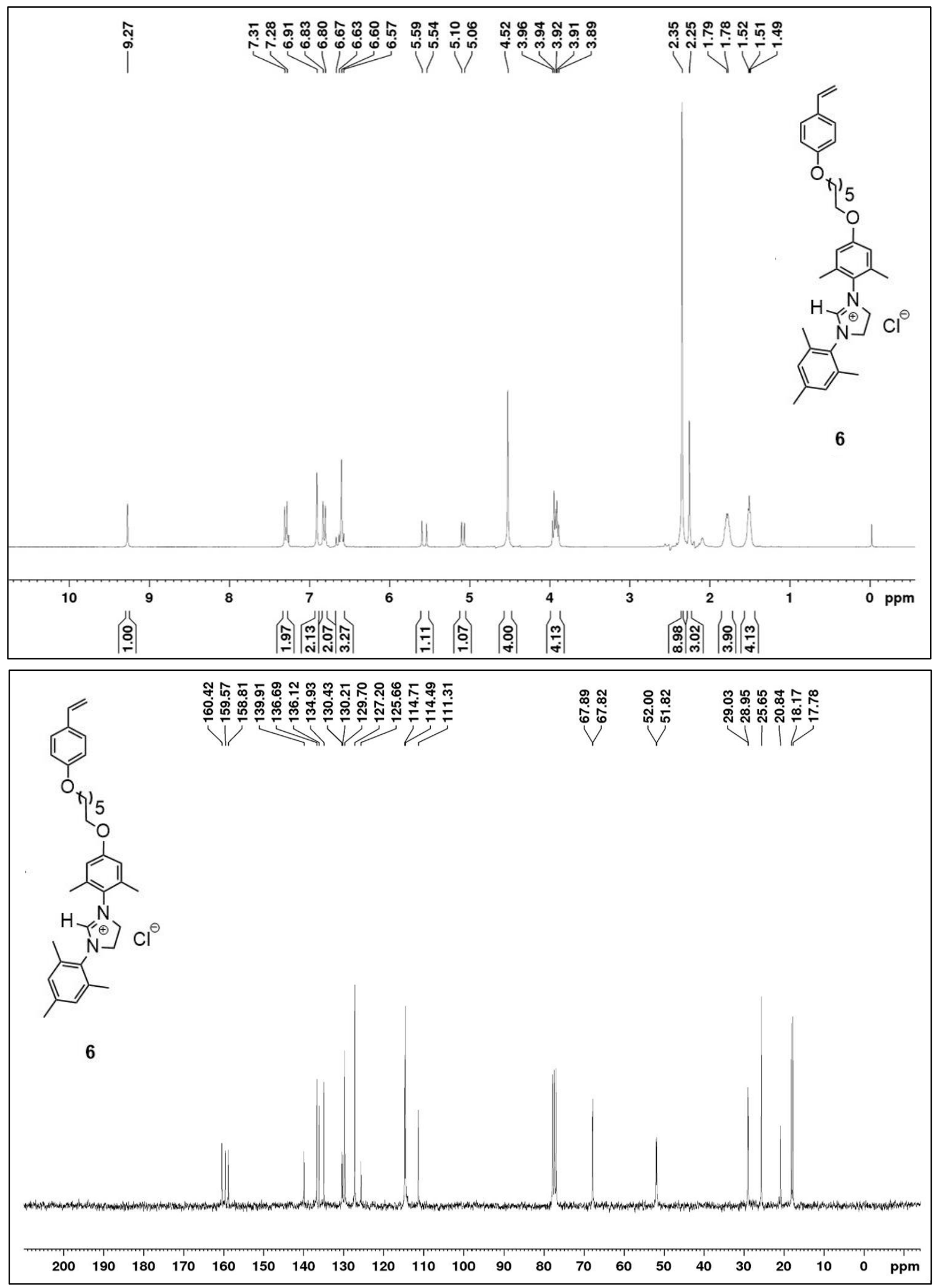

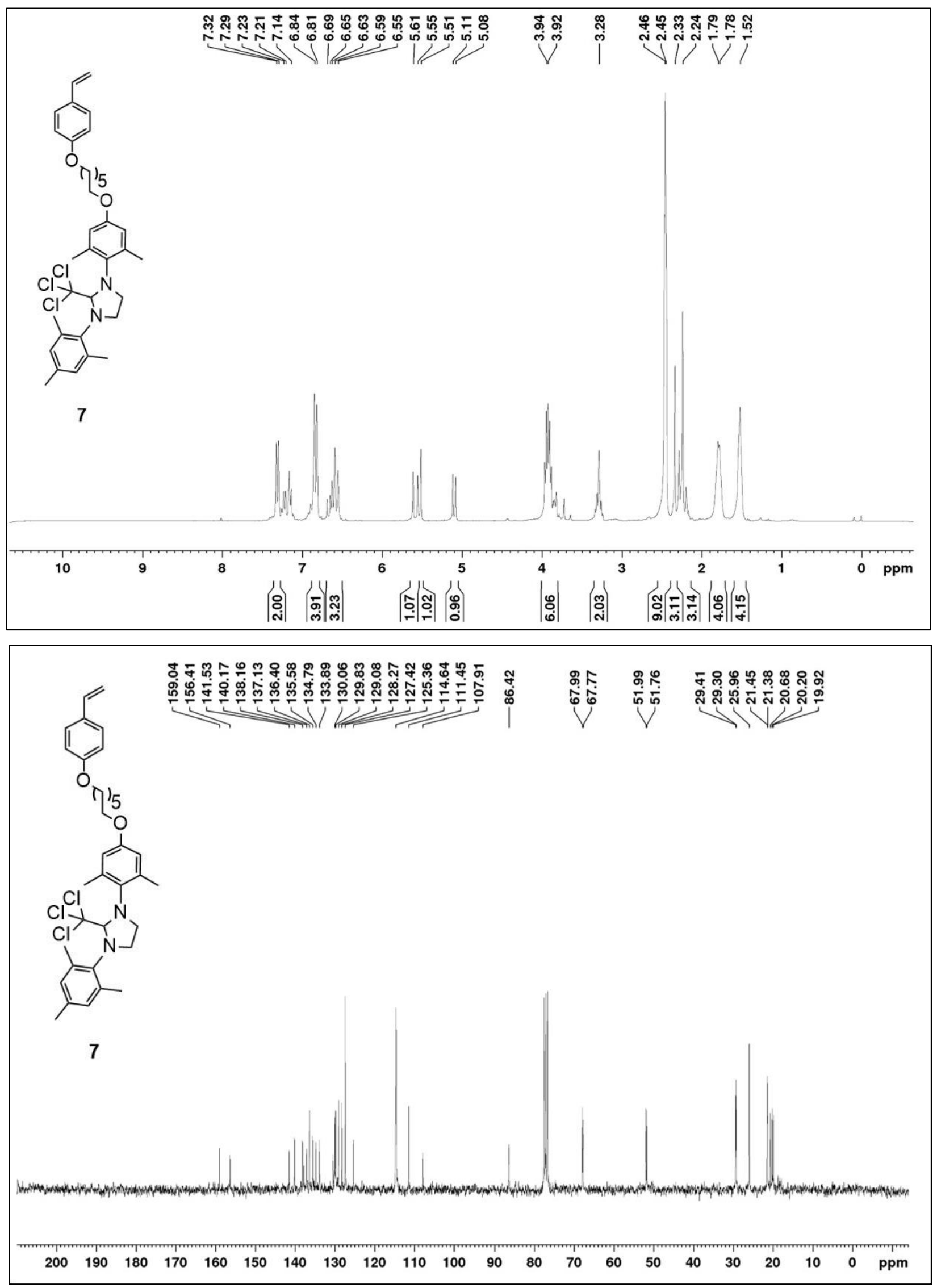


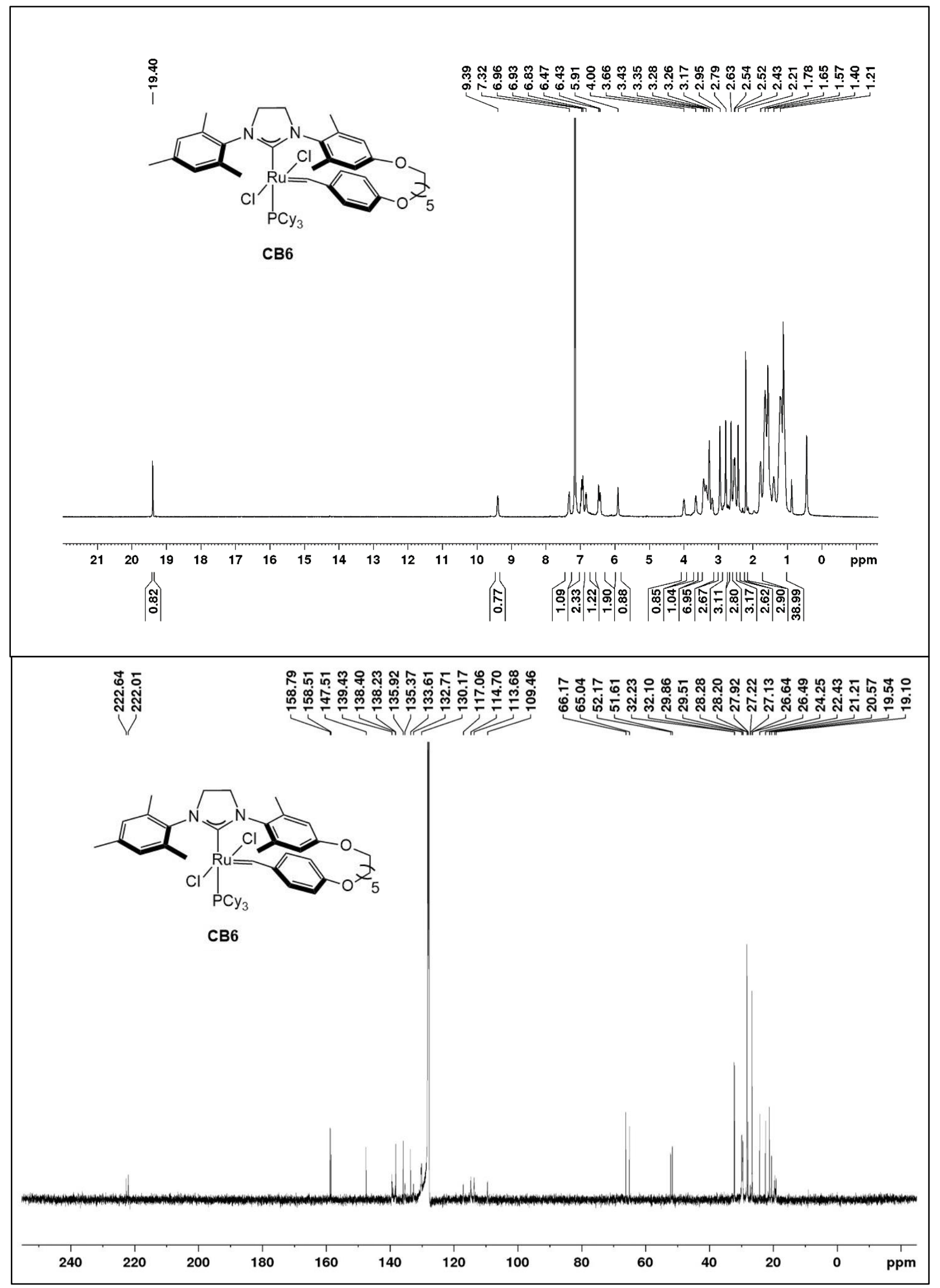




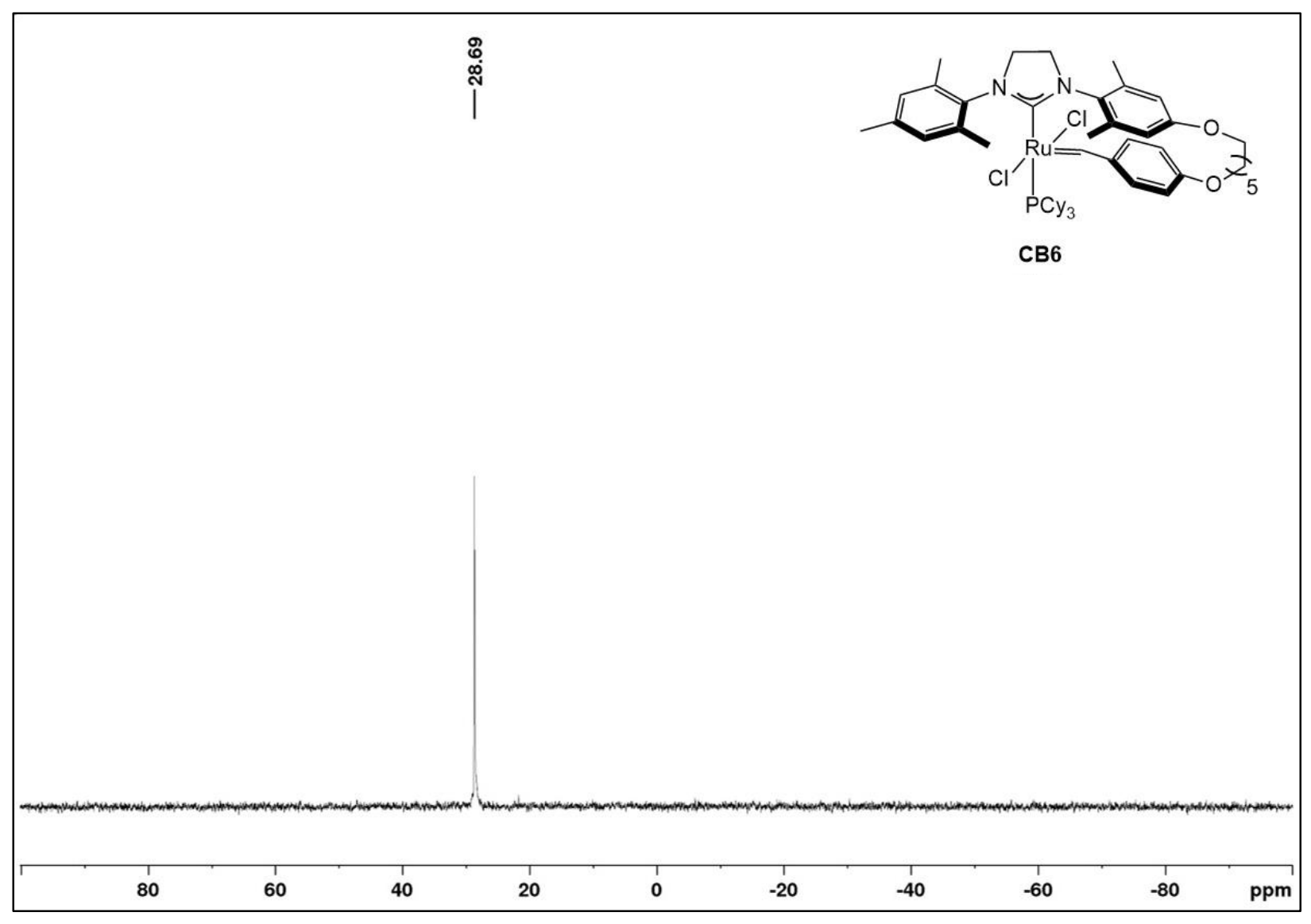



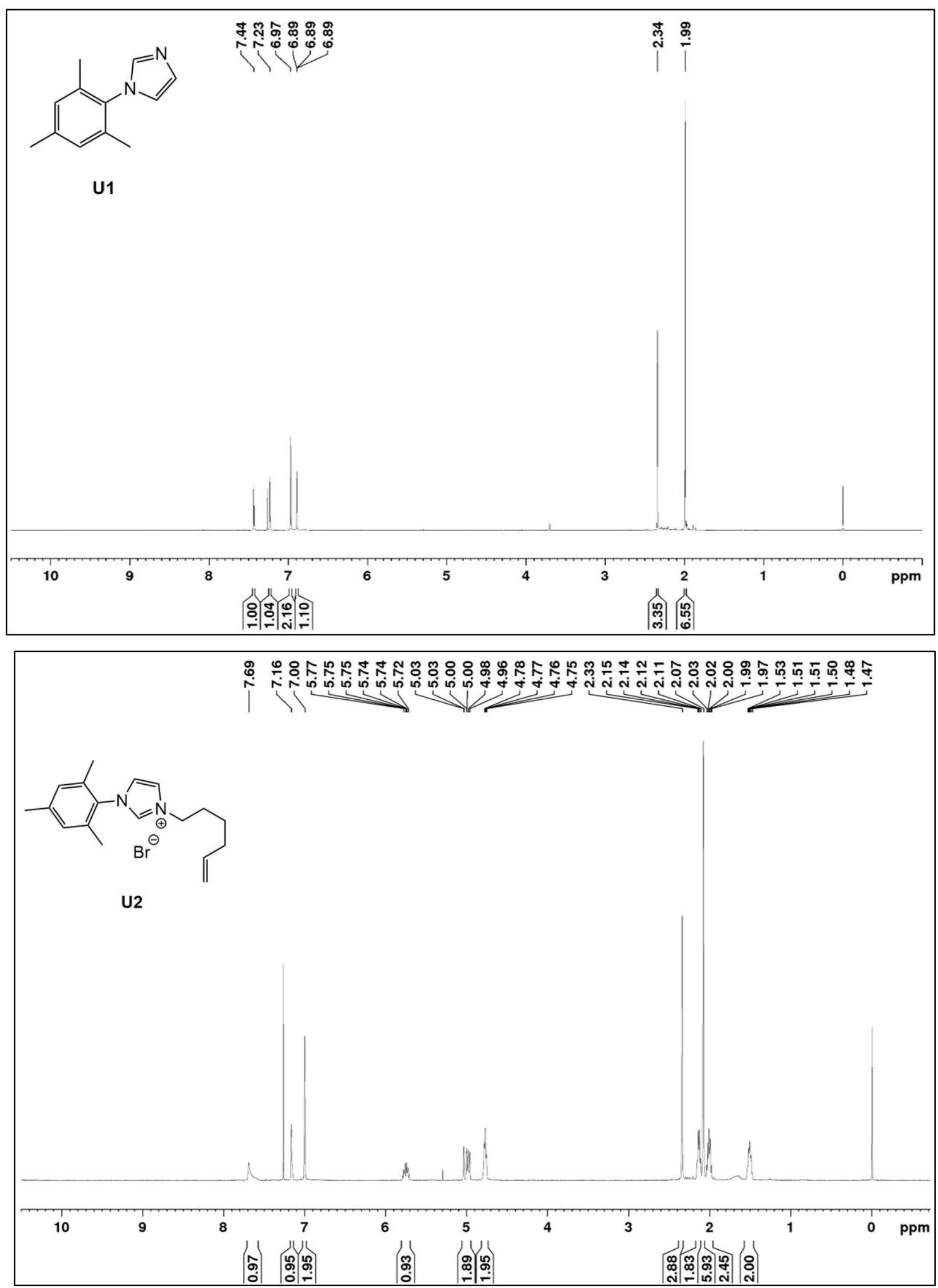


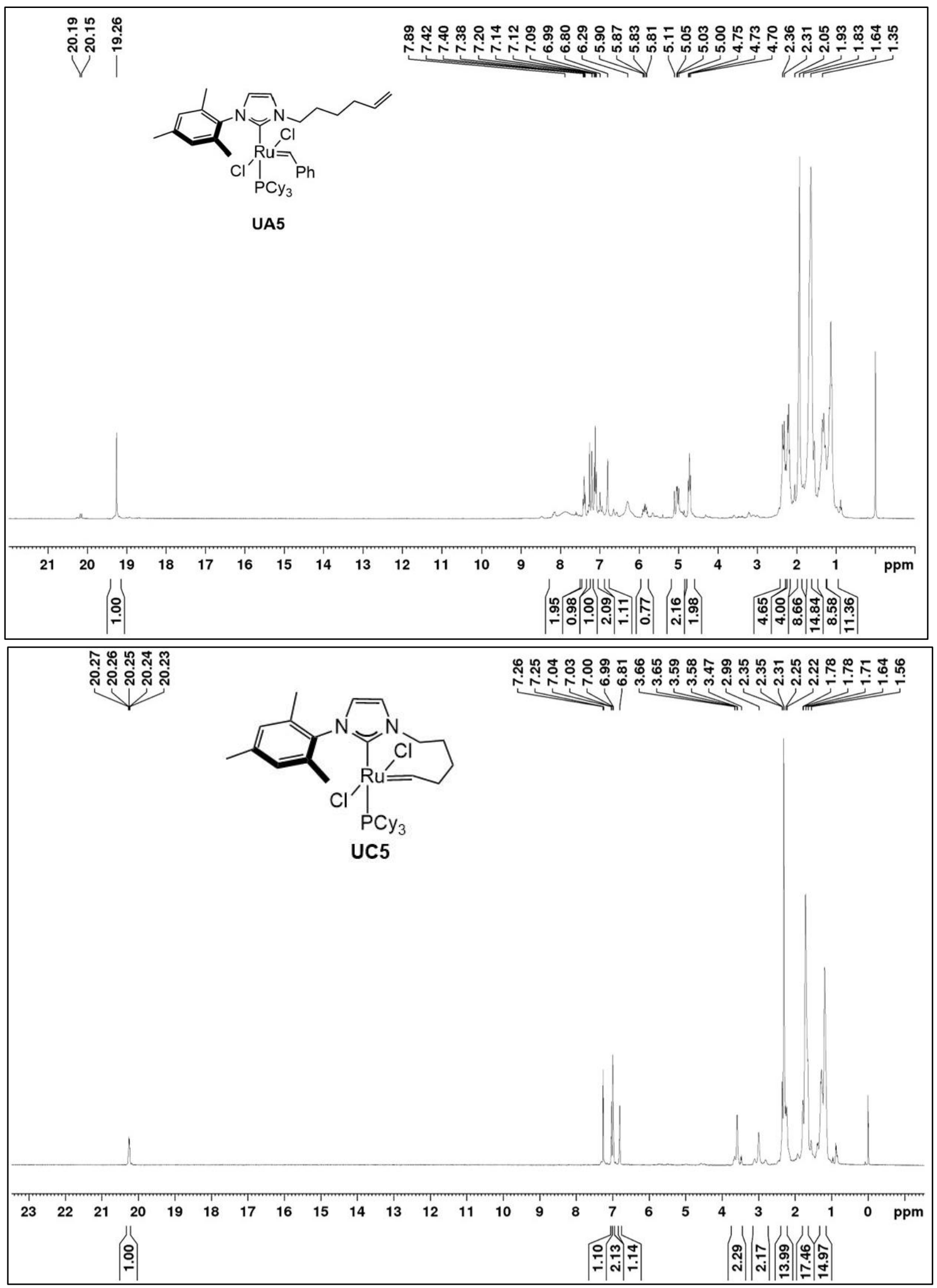



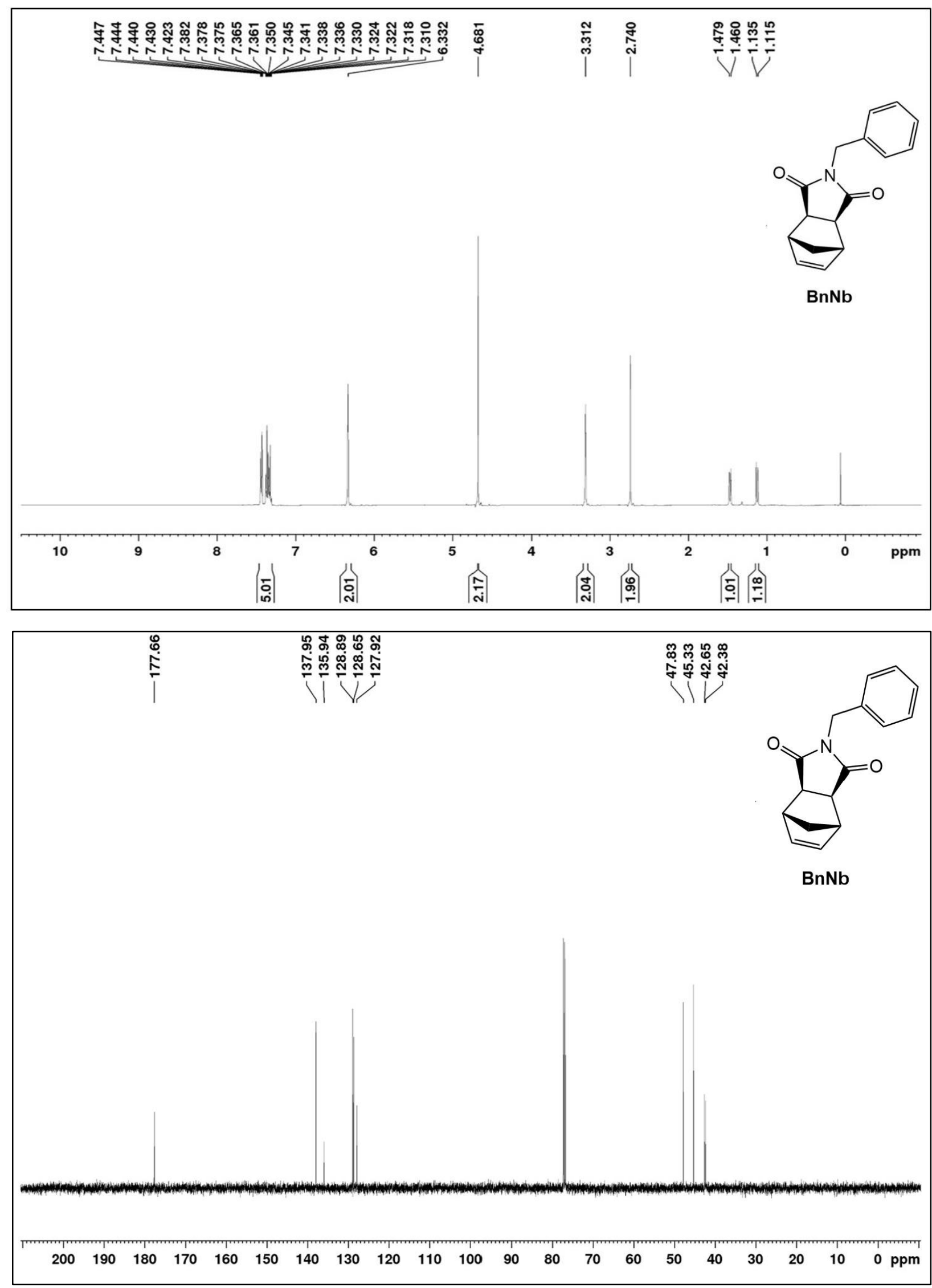


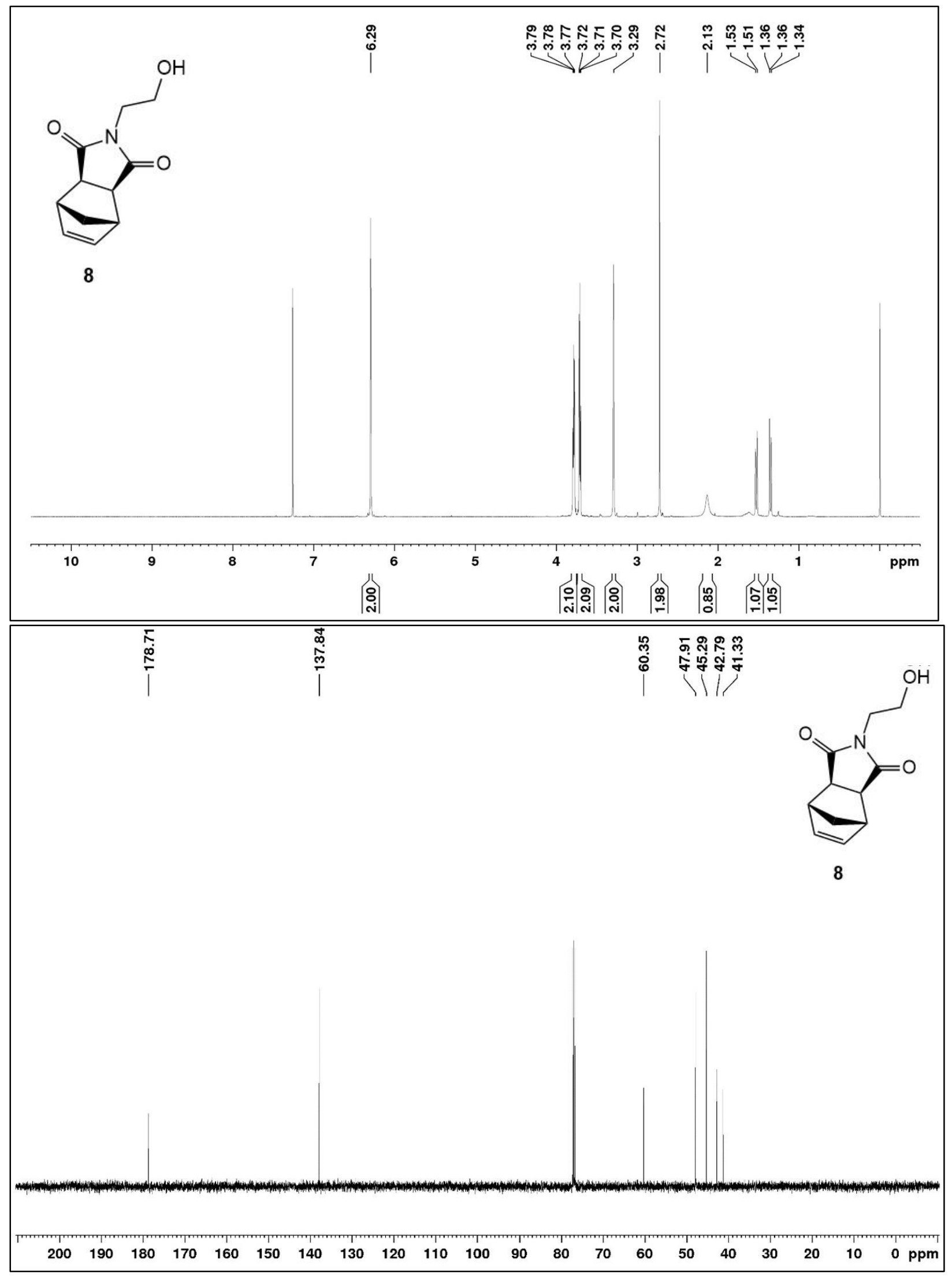



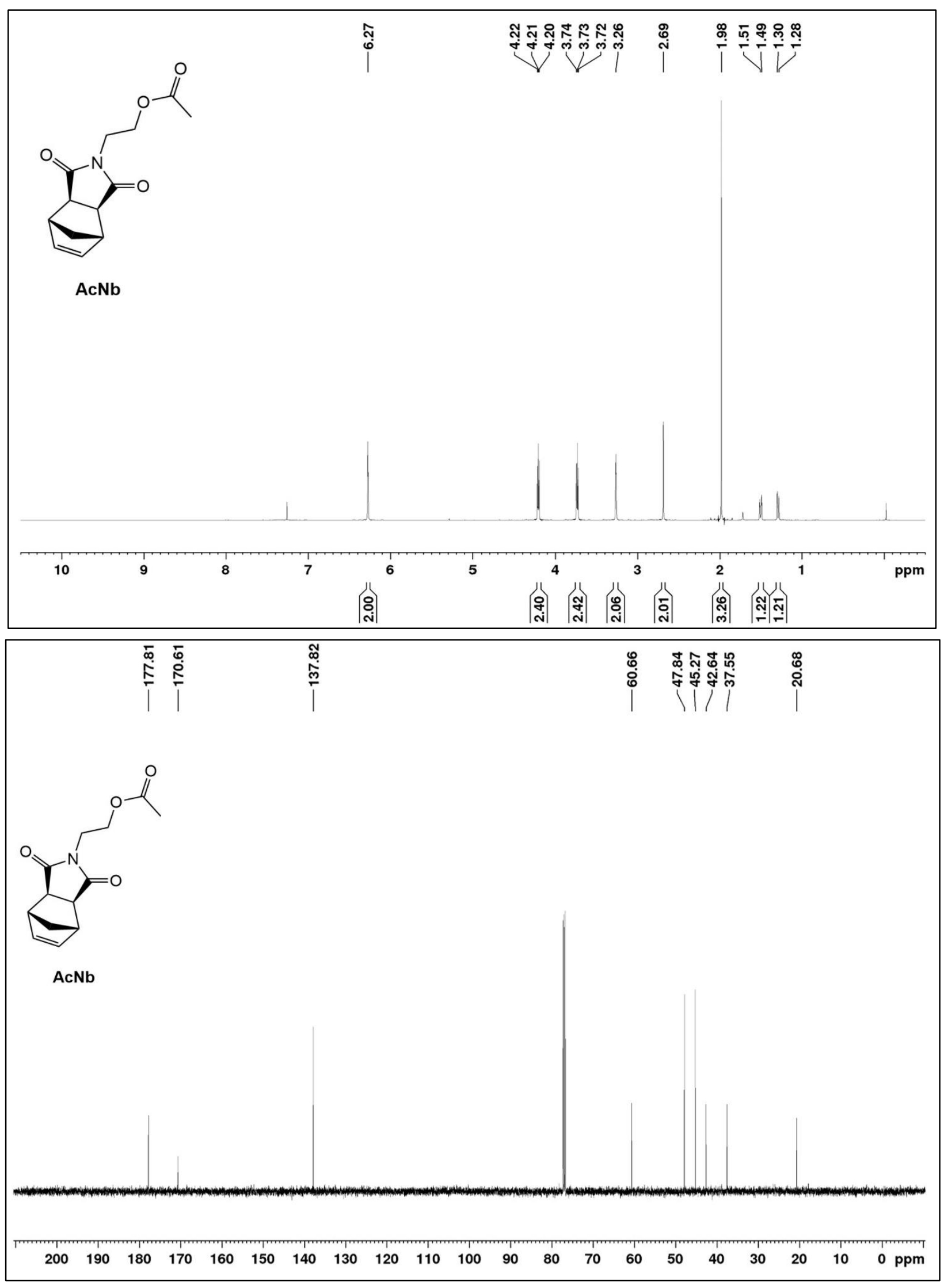


\section{$\underline{\text { X-ray Crystallography }}$}

Table S1. Crystallographic data and structure refinement for CB6.

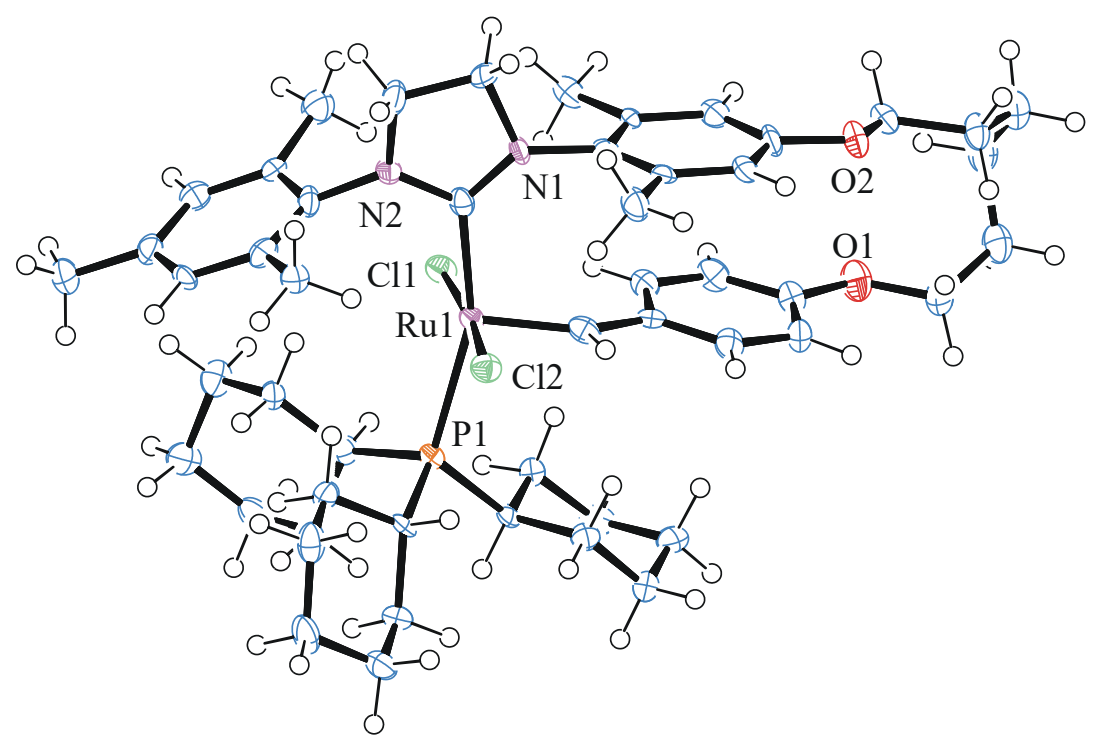

Empirical formula

C51 H73 Cl2 N2 O2 P Ru

Formula weight

949.05

Temperature

100(2) K

Wavelength

$0.71073 \AA$

Crystal system

Triclinic

Space group

P -1

Unit cell dimensions

$a=12.4126(6) \AA$

$\mathrm{a}=90.475(3)^{\circ}$.

$\mathrm{b}=12.9043(6) \AA$

$\mathrm{b}=94.766(2)^{\circ}$.

$\mathrm{c}=14.7041(8) \AA$

$\mathrm{g}=96.207(2)^{\circ}$.

Volume

$2333.0(2) \AA^{3}$

Z

2

Density (calculated)

$1.351 \mathrm{Mg} / \mathrm{m}^{3}$

Absorption coefficient

$0.527 \mathrm{~mm}^{-1}$

$\mathrm{F}(000)$

1004 


$\begin{array}{ll}\text { Crystal size } & 0.120 \times 0.070 \times 0.030 \mathrm{~mm}^{3} \\ \text { Theta range for data collection } & 2.070 \text { to } 25.027^{\circ} . \\ \text { Index ranges } & -14<=\mathrm{h}<=14,-15<=\mathrm{k}<=15,-17<=\mathrm{l}<=17 \\ \text { Reflections collected } & 16426 \\ \text { Independent reflections } & 8246[\mathrm{R}(\mathrm{int})=0.1048] \\ \text { Completeness to theta }=25.000^{\circ} & 99.9 \% \\ \text { Refinement method } & \text { Full-matrix least-squares on } \mathrm{F}^{2} \\ \text { Data / restraints / parameters } & 8246 / 0 / 537 \\ \text { Goodness-of-fit on } \mathrm{F}^{2} & 0.972 \\ \text { Final R indices }[\mathrm{I}>2 \text { sigma(I)] } & \mathrm{R} 1=0.0531, \mathrm{wR} 2=0.0890 \\ \text { R indices (all data) } & \mathrm{R} 1=0.1297, \mathrm{wR} 2=0.1110 \\ \text { Largest diff. peak and hole } & 0.547 \text { and }-0.634 \text { e. } \AA^{-3}\end{array}$

Table S2. Atomic coordinates $\left(\mathrm{x} 10^{4}\right)$ and equivalent isotropic displacement parameters $\left(\AA^{2} \times 10^{3}\right)$ for CB6. $U($ eq $)$ is defined as one third of the trace of the orthogonalized $U^{\mathrm{ij}}$ tensor.

\begin{tabular}{lrrrr}
\hline & $\mathrm{x}$ & $\mathrm{y}$ & $\mathrm{z}$ & $\mathrm{U}(\mathrm{eq})$ \\
\hline $\mathrm{C}(2)$ & $11059(4)$ & $1736(4)$ & $2453(3)$ & $19(1)$ \\
$\mathrm{C}(3)$ & $11117(4)$ & $815(4)$ & $1972(3)$ & $19(1)$ \\
$\mathrm{C}(4)$ & $11580(4)$ & $2(4)$ & $2423(4)$ & $24(1)$ \\
$\mathrm{C}(5)$ & $11951(4)$ & $120(4)$ & $3342(4)$ & $23(1)$ \\
$\mathrm{C}(6)$ & $11897(4)$ & $1044(4)$ & $3794(3)$ & $22(1)$ \\
$\mathrm{C}(7)$ & $11462(4)$ & $1866(4)$ & $3375(3)$ & $19(1)$ \\
$\mathrm{C}(8)$ & $11423(4)$ & $2887(4)$ & $3871(3)$ & $29(1)$ \\
$\mathrm{C}(9)$ & $10681(4)$ & $672(4)$ & $990(3)$ & $27(1)$ \\
$\mathrm{C}(10)$ & $9590(4)$ & $2701(4)$ & $1710(3)$ & $20(1)$ \\
$\mathrm{C}(11)$ & $11408(4)$ & $3320(4)$ & $1518(3)$ & $26(1)$ \\
$\mathrm{C}(12)$ & $10672(4)$ & $4072(4)$ & $1077(4)$ & $37(2)$ \\
$\mathrm{C}(13)$ & $8660(4)$ & $3838(4)$ & $615(3)$ & $21(1)$ \\
$\mathrm{C}(14)$ & $8131(4)$ & $4666(4)$ & $933(3)$ & $24(1)$
\end{tabular}




\begin{tabular}{|c|c|c|c|c|}
\hline$C(15)$ & $7268(4)$ & 4987(4) & $373(4)$ & $23(1)$ \\
\hline$C(16)$ & $6962(4)$ & $4556(4)$ & $-487(4)$ & $24(1)$ \\
\hline $\mathrm{C}(17)$ & $7526(4)$ & $3769(4)$ & $-794(3)$ & $24(1)$ \\
\hline $\mathrm{C}(18)$ & $8394(4)$ & $3413(4)$ & $-267(3)$ & $22(1)$ \\
\hline$C(19)$ & $9038(4)$ & $2623(4)$ & $-652(4)$ & $33(2)$ \\
\hline$C(20)$ & $8505(4)$ & $5228(4)$ & $1816(3)$ & $27(1)$ \\
\hline$C(21)$ & $6035(4)$ & $4928(4)$ & $-1070(4)$ & $36(2)$ \\
\hline$C(22)$ & $8685(4)$ & $1070(4)$ & 2987(3) & $23(1)$ \\
\hline$C(23)$ & $8895(4)$ & $-16(4)$ & $3023(3)$ & $21(1)$ \\
\hline$C(24)$ & $8785(4)$ & $-735(4)$ & $2276(4)$ & $26(1)$ \\
\hline$C(25)$ & $9028(4)$ & $-1735(4)$ & 2401(4) & $28(1)$ \\
\hline$C(26)$ & $9407(4)$ & $-2086(4)$ & $3254(4)$ & $25(1)$ \\
\hline$C(27)$ & $9469(4)$ & $-1418(4)$ & $4005(4)$ & $29(1)$ \\
\hline C(28) & $9225(4)$ & $-394(4)$ & $3875(4)$ & $25(1)$ \\
\hline C(29) & 10174(4) & $-3447(4)$ & $4142(4)$ & $32(2)$ \\
\hline$C(30)$ & 10821(4) & $-4329(4)$ & $3918(4)$ & $33(2)$ \\
\hline$C(31)$ & $11727(5)$ & $-4056(4)$ & $3314(4)$ & $33(2)$ \\
\hline C(32) & $12777(4)$ & $-3494(4)$ & $3763(4)$ & $34(2)$ \\
\hline$C(33)$ & $12763(4)$ & $-2397(4)$ & $4130(3)$ & $27(1)$ \\
\hline$C(34)$ & $12354(4)$ & $-1664(4)$ & $3421(3)$ & $24(1)$ \\
\hline C(35) & $5795(4)$ & $2709(3)$ & $3173(3)$ & $16(1)$ \\
\hline$C(36)$ & $5884(4)$ & $3784(4)$ & $2724(3)$ & $23(1)$ \\
\hline$C(37)$ & $5731(4)$ & $4652(4)$ & $3402(3)$ & $30(1)$ \\
\hline$C(38)$ & $4642(5)$ & $4416(4)$ & $3800(4)$ & $34(2)$ \\
\hline$C(39)$ & $4533(4)$ & $3368(4)$ & $4238(4)$ & $31(2)$ \\
\hline $\mathrm{C}(40)$ & $4699(4)$ & 2494(4) & $3576(3)$ & $24(1)$ \\
\hline$C(41)$ & $5373(4)$ & $1633(4)$ & $1326(3)$ & $21(1)$ \\
\hline$C(42)$ & $5652(4)$ & $2502(4)$ & $650(3)$ & $23(1)$ \\
\hline C(43) & $5100(4)$ & $2229(4)$ & $-292(3)$ & $29(1)$ \\
\hline$C(44)$ & $3860(4)$ & $2017(4)$ & $-273(3)$ & $30(1)$ \\
\hline$C(45)$ & $3583(4)$ & $1186(4)$ & $424(3)$ & $31(1)$ \\
\hline$C(46)$ & $4135(4)$ & $1472(4)$ & $1375(3)$ & $28(1)$ \\
\hline$C(47)$ & $5735(4)$ & $443(4)$ & $2937(3)$ & $19(1)$ \\
\hline$C(48)$ & $6254(4)$ & $356(4)$ & $3922(3)$ & $22(1)$ \\
\hline $\mathrm{C}(49)$ & $5822(4)$ & $-674(4)$ & $4324(3)$ & $25(1)$ \\
\hline$C(50)$ & $6057(4)$ & $-1601(4)$ & $3752(3)$ & $31(2)$ \\
\hline
\end{tabular}




\begin{tabular}{lrrrr}
$\mathrm{C}(51)$ & $5525(4)$ & $-1535(4)$ & $2781(3)$ & $28(1)$ \\
$\mathrm{C}(52)$ & $5903(4)$ & $-499(4)$ & $2345(3)$ & $22(1)$ \\
$\mathrm{N}(1)$ & $10632(3)$ & $2598(3)$ & $1982(3)$ & $20(1)$ \\
$\mathrm{N}(2)$ & $9591(3)$ & $3508(3)$ & $1147(3)$ & $22(1)$ \\
$\mathrm{O}(1)$ & $9700(3)$ & $-3069(3)$ & $3289(2)$ & $37(1)$ \\
$\mathrm{O}(2)$ & $12397(3)$ & $-654(3)$ & $3841(2)$ & $27(1)$ \\
$\mathrm{P}(1)$ & $6221(1)$ & $1696(1)$ & $2421(1)$ & $19(1)$ \\
$\mathrm{Cl}(1)$ & $7769(1)$ & $944(1)$ & $827(1)$ & $24(1)$ \\
$\mathrm{Cl}(2)$ & $8486(1)$ & $3254(1)$ & $3432(1)$ & $24(1)$ \\
$\mathrm{Ru}(1)$ & $8160(1)$ & $2005(1)$ & $2165(1)$ & $18(1)$ \\
& & & & \\
\hline
\end{tabular}

Table S3. Bond lengths $[\AA]$ and angles $\left[{ }^{\circ}\right]$ for CB6.

\begin{tabular}{ll}
\hline $\mathrm{C}(2)-\mathrm{C}(3)$ & $1.390(7)$ \\
$\mathrm{C}(2)-\mathrm{C}(7)$ & $1.407(7)$ \\
$\mathrm{C}(2)-\mathrm{N}(1)$ & $1.442(6)$ \\
$\mathrm{C}(3)-\mathrm{C}(4)$ & $1.396(6)$ \\
$\mathrm{C}(3)-\mathrm{C}(9)$ & $1.502(6)$ \\
$\mathrm{C}(4)-\mathrm{C}(5)$ & $1.393(7)$ \\
$\mathrm{C}(4)-\mathrm{H}(4)$ & 0.9500 \\
$\mathrm{C}(5)-\mathrm{C}(6)$ & $1.371(7)$ \\
$\mathrm{C}(5)-\mathrm{O}(2)$ & $1.381(5)$ \\
$\mathrm{C}(6)-\mathrm{C}(7)$ & $1.370(6)$ \\
$\mathrm{C}(6)-\mathrm{H}(6)$ & 0.9500 \\
$\mathrm{C}(7)-\mathrm{C}(8)$ & $1.508(7)$ \\
$\mathrm{C}(8)-\mathrm{H}(8 \mathrm{~A})$ & 0.9800 \\
$\mathrm{C}(8)-\mathrm{H}(8 \mathrm{~B})$ & 0.9800 \\
$\mathrm{C}(8)-\mathrm{H}(8 \mathrm{C})$ & 0.9800 \\
$\mathrm{C}(9)-\mathrm{H}(9 \mathrm{~A})$ & 0.9800 \\
$\mathrm{C}(9)-\mathrm{H}(9 \mathrm{~B})$ & 0.9800 \\
$\mathrm{C}(9)-\mathrm{H}(9 \mathrm{C})$ & 0.9800 \\
$\mathrm{C}(10)-\mathrm{N}(2)$ & $1.336(5)$ \\
$\mathrm{C}(10)-\mathrm{N}(1)$ & $1.343(6)$ \\
$\mathrm{C}(10)-\mathrm{Ru}(1)$ & $2.065(5)$ \\
$\mathrm{C}(11)-\mathrm{N}(1)$ & $1.479(6)$ \\
&
\end{tabular}




\begin{tabular}{|c|c|}
\hline$C(11)-C(12)$ & $1.516(6)$ \\
\hline $\mathrm{C}(11)-\mathrm{H}(11 \mathrm{~A})$ & 0.9900 \\
\hline $\mathrm{C}(11)-\mathrm{H}(11 \mathrm{~B})$ & 0.9900 \\
\hline $\mathrm{C}(12)-\mathrm{N}(2)$ & $1.468(6)$ \\
\hline $\mathrm{C}(12)-\mathrm{H}(12 \mathrm{~A})$ & 0.9900 \\
\hline $\mathrm{C}(12)-\mathrm{H}(12 \mathrm{~B})$ & 0.9900 \\
\hline$C(13)-C(18)$ & $1.403(7)$ \\
\hline$C(13)-C(14)$ & $1.409(7)$ \\
\hline $\mathrm{C}(13)-\mathrm{N}(2)$ & $1.447(6)$ \\
\hline$C(14)-C(15)$ & $1.397(6)$ \\
\hline$C(14)-C(20)$ & $1.497(7)$ \\
\hline$C(15)-C(16)$ & $1.384(7)$ \\
\hline $\mathrm{C}(15)-\mathrm{H}(15)$ & 0.9500 \\
\hline$C(16)-C(17)$ & $1.387(7)$ \\
\hline$C(16)-C(21)$ & $1.500(6)$ \\
\hline$C(17)-C(18)$ & $1.395(6)$ \\
\hline $\mathrm{C}(17)-\mathrm{H}(17)$ & 0.9500 \\
\hline$C(18)-C(19)$ & $1.496(7)$ \\
\hline $\mathrm{C}(19)-\mathrm{H}(19 \mathrm{~A})$ & 0.9800 \\
\hline $\mathrm{C}(19)-\mathrm{H}(19 \mathrm{~B})$ & 0.9800 \\
\hline $\mathrm{C}(19)-\mathrm{H}(19 \mathrm{C})$ & 0.9800 \\
\hline $\mathrm{C}(20)-\mathrm{H}(20 \mathrm{~A})$ & 0.9800 \\
\hline $\mathrm{C}(20)-\mathrm{H}(20 \mathrm{~B})$ & 0.9800 \\
\hline $\mathrm{C}(20)-\mathrm{H}(20 \mathrm{C})$ & 0.9800 \\
\hline $\mathrm{C}(21)-\mathrm{H}(21 \mathrm{~A})$ & 0.9800 \\
\hline $\mathrm{C}(21)-\mathrm{H}(21 \mathrm{~B})$ & 0.9800 \\
\hline $\mathrm{C}(21)-\mathrm{H}(21 \mathrm{C})$ & 0.9800 \\
\hline$C(22)-C(23)$ & $1.453(6)$ \\
\hline $\mathrm{C}(22)-\mathrm{Ru}(1)$ & $1.846(5)$ \\
\hline $\mathrm{C}(22)-\mathrm{H}(22)$ & 0.9500 \\
\hline$C(23)-C(28)$ & $1.394(6)$ \\
\hline$C(23)-C(24)$ & $1.420(7)$ \\
\hline$C(24)-C(25)$ & $1.367(7)$ \\
\hline $\mathrm{C}(24)-\mathrm{H}(24)$ & 0.9500 \\
\hline$C(25)-C(26)$ & $1.399(7)$ \\
\hline $\mathrm{C}(25)-\mathrm{H}(25)$ & 0.9500 \\
\hline
\end{tabular}




\begin{tabular}{|c|c|}
\hline $\mathrm{C}(26)-\mathrm{O}(1)$ & $1.357(6)$ \\
\hline$C(26)-C(27)$ & $1.387(7)$ \\
\hline$C(27)-C(28)$ & $1.399(7)$ \\
\hline $\mathrm{C}(27)-\mathrm{H}(27)$ & 0.9500 \\
\hline $\mathrm{C}(28)-\mathrm{H}(28)$ & 0.9500 \\
\hline $\mathrm{C}(29)-\mathrm{O}(1)$ & $1.451(5)$ \\
\hline$C(29)-C(30)$ & $1.511(7)$ \\
\hline $\mathrm{C}(29)-\mathrm{H}(29 \mathrm{~A})$ & 0.9900 \\
\hline $\mathrm{C}(29)-\mathrm{H}(29 \mathrm{~B})$ & 0.9900 \\
\hline$C(30)-C(31)$ & $1.504(7)$ \\
\hline $\mathrm{C}(30)-\mathrm{H}(30 \mathrm{~A})$ & 0.9900 \\
\hline $\mathrm{C}(30)-\mathrm{H}(30 \mathrm{~B})$ & 0.9900 \\
\hline C(31)-C(32) & $1.519(7)$ \\
\hline $\mathrm{C}(31)-\mathrm{H}(31 \mathrm{~A})$ & 0.9900 \\
\hline $\mathrm{C}(31)-\mathrm{H}(31 \mathrm{~B})$ & 0.9900 \\
\hline$C(32)-C(33)$ & $1.513(7)$ \\
\hline $\mathrm{C}(32)-\mathrm{H}(32 \mathrm{~A})$ & 0.9900 \\
\hline $\mathrm{C}(32)-\mathrm{H}(32 \mathrm{~B})$ & 0.9900 \\
\hline$C(33)-C(34)$ & $1.508(6)$ \\
\hline $\mathrm{C}(33)-\mathrm{H}(33 \mathrm{~A})$ & 0.9900 \\
\hline $\mathrm{C}(33)-\mathrm{H}(33 \mathrm{~B})$ & 0.9900 \\
\hline $\mathrm{C}(34)-\mathrm{O}(2)$ & $1.431(5)$ \\
\hline $\mathrm{C}(34)-\mathrm{H}(34 \mathrm{~A})$ & 0.9900 \\
\hline $\mathrm{C}(34)-\mathrm{H}(34 \mathrm{~B})$ & 0.9900 \\
\hline$C(35)-C(40)$ & $1.527(6)$ \\
\hline$C(35)-C(36)$ & $1.538(6)$ \\
\hline$C(35)-P(1)$ & $1.853(5)$ \\
\hline $\mathrm{C}(35)-\mathrm{H}(35)$ & 1.0000 \\
\hline$C(36)-C(37)$ & $1.531(7)$ \\
\hline $\mathrm{C}(36)-\mathrm{H}(36 \mathrm{~A})$ & 0.9900 \\
\hline $\mathrm{C}(36)-\mathrm{H}(36 \mathrm{~B})$ & 0.9900 \\
\hline $\mathrm{C}(37)-\mathrm{C}(38)$ & $1.519(7)$ \\
\hline $\mathrm{C}(37)-\mathrm{H}(37 \mathrm{~A})$ & 0.9900 \\
\hline $\mathrm{C}(37)-\mathrm{H}(37 \mathrm{~B})$ & 0.9900 \\
\hline$C(38)-C(39)$ & $1.501(7)$ \\
\hline $\mathrm{C}(38)-\mathrm{H}(38 \mathrm{~A})$ & 0.9900 \\
\hline
\end{tabular}




\begin{tabular}{|c|c|}
\hline $\mathrm{C}(38)-\mathrm{H}(38 \mathrm{~B})$ & 0.9900 \\
\hline$C(39)-C(40)$ & $1.526(7)$ \\
\hline $\mathrm{C}(39)-\mathrm{H}(39 \mathrm{~A})$ & 0.9900 \\
\hline $\mathrm{C}(39)-\mathrm{H}(39 \mathrm{~B})$ & 0.9900 \\
\hline $\mathrm{C}(40)-\mathrm{H}(40 \mathrm{~A})$ & 0.9900 \\
\hline $\mathrm{C}(40)-\mathrm{H}(40 \mathrm{~B})$ & 0.9900 \\
\hline$C(41)-C(46)$ & $1.535(6)$ \\
\hline$C(41)-C(42)$ & $1.535(6)$ \\
\hline $\mathrm{C}(41)-\mathrm{P}(1)$ & $1.845(5)$ \\
\hline $\mathrm{C}(41)-\mathrm{H}(41)$ & 1.0000 \\
\hline$C(42)-C(43)$ & $1.514(6)$ \\
\hline $\mathrm{C}(42)-\mathrm{H}(42 \mathrm{~A})$ & 0.9900 \\
\hline $\mathrm{C}(42)-\mathrm{H}(42 \mathrm{~B})$ & 0.9900 \\
\hline$C(43)-C(44)$ & $1.537(7)$ \\
\hline $\mathrm{C}(43)-\mathrm{H}(43 \mathrm{~A})$ & 0.9900 \\
\hline $\mathrm{C}(43)-\mathrm{H}(43 \mathrm{~B})$ & 0.9900 \\
\hline$C(44)-C(45)$ & $1.522(7)$ \\
\hline $\mathrm{C}(44)-\mathrm{H}(44 \mathrm{~A})$ & 0.9900 \\
\hline $\mathrm{C}(44)-\mathrm{H}(44 \mathrm{~B})$ & 0.9900 \\
\hline$C(45)-C(46)$ & $1.529(7)$ \\
\hline $\mathrm{C}(45)-\mathrm{H}(45 \mathrm{~A})$ & 0.9900 \\
\hline $\mathrm{C}(45)-\mathrm{H}(45 \mathrm{~B})$ & 0.9900 \\
\hline $\mathrm{C}(46)-\mathrm{H}(46 \mathrm{~A})$ & 0.9900 \\
\hline $\mathrm{C}(46)-\mathrm{H}(46 \mathrm{~B})$ & 0.9900 \\
\hline$C(47)-C(52)$ & $1.532(7)$ \\
\hline$C(47)-C(48)$ & $1.545(6)$ \\
\hline $\mathrm{C}(47)-\mathrm{P}(1)$ & $1.854(5)$ \\
\hline $\mathrm{C}(47)-\mathrm{H}(47)$ & 1.0000 \\
\hline$C(48)-C(49)$ & $1.521(6)$ \\
\hline $\mathrm{C}(48)-\mathrm{H}(48 \mathrm{~A})$ & 0.9900 \\
\hline $\mathrm{C}(48)-\mathrm{H}(48 \mathrm{~B})$ & 0.9900 \\
\hline$C(49)-C(50)$ & $1.523(7)$ \\
\hline $\mathrm{C}(49)-\mathrm{H}(49 \mathrm{~A})$ & 0.9900 \\
\hline $\mathrm{C}(49)-\mathrm{H}(49 \mathrm{~B})$ & 0.9900 \\
\hline$C(50)-C(51)$ & $1.529(7)$ \\
\hline $\mathrm{C}(50)-\mathrm{H}(50 \mathrm{~A})$ & 0.9900 \\
\hline
\end{tabular}




\begin{tabular}{|c|c|}
\hline $\mathrm{C}(50)-\mathrm{H}(50 \mathrm{~B})$ & 0.9900 \\
\hline$C(51)-C(52)$ & $1.532(6)$ \\
\hline $\mathrm{C}(51)-\mathrm{H}(51 \mathrm{~A})$ & 0.9900 \\
\hline $\mathrm{C}(51)-\mathrm{H}(51 \mathrm{~B})$ & 0.9900 \\
\hline $\mathrm{C}(52)-\mathrm{H}(52 \mathrm{~A})$ & 0.9900 \\
\hline $\mathrm{C}(52)-\mathrm{H}(52 \mathrm{~B})$ & 0.9900 \\
\hline $\mathrm{P}(1)-\mathrm{Ru}(1)$ & $2.4583(14)$ \\
\hline $\mathrm{Cl}(1)-\mathrm{Ru}(1)$ & $2.3742(14)$ \\
\hline $\mathrm{Cl}(2)-\mathrm{Ru}(1)$ & $2.4315(14)$ \\
\hline$C(3)-C(2)-C(7)$ & $121.5(4)$ \\
\hline$C(3)-C(2)-N(1)$ & 119.1(4) \\
\hline $\mathrm{C}(7)-\mathrm{C}(2)-\mathrm{N}(1)$ & $119.3(5)$ \\
\hline$C(2)-C(3)-C(4)$ & $118.7(5)$ \\
\hline$C(2)-C(3)-C(9)$ & $121.6(4)$ \\
\hline$C(4)-C(3)-C(9)$ & $119.7(5)$ \\
\hline$C(5)-C(4)-C(3)$ & $119.8(5)$ \\
\hline $\mathrm{C}(5)-\mathrm{C}(4)-\mathrm{H}(4)$ & 120.1 \\
\hline $\mathrm{C}(3)-\mathrm{C}(4)-\mathrm{H}(4)$ & 120.1 \\
\hline $\mathrm{C}(6)-\mathrm{C}(5)-\mathrm{O}(2)$ & $116.6(5)$ \\
\hline$C(6)-C(5)-C(4)$ & $120.1(4)$ \\
\hline $\mathrm{O}(2)-\mathrm{C}(5)-\mathrm{C}(4)$ & $123.3(5)$ \\
\hline$C(7)-C(6)-C(5)$ & $122.0(5)$ \\
\hline$C(7)-C(6)-H(6)$ & 119.0 \\
\hline$C(5)-C(6)-H(6)$ & 119.0 \\
\hline$C(6)-C(7)-C(2)$ & $117.8(5)$ \\
\hline$C(6)-C(7)-C(8)$ & $121.7(5)$ \\
\hline$C(2)-C(7)-C(8)$ & $120.4(4)$ \\
\hline$C(7)-C(8)-H(8 A)$ & 109.5 \\
\hline $\mathrm{C}(7)-\mathrm{C}(8)-\mathrm{H}(8 \mathrm{~B})$ & 109.5 \\
\hline $\mathrm{H}(8 \mathrm{~A})-\mathrm{C}(8)-\mathrm{H}(8 \mathrm{~B})$ & 109.5 \\
\hline $\mathrm{C}(7)-\mathrm{C}(8)-\mathrm{H}(8 \mathrm{C})$ & 109.5 \\
\hline $\mathrm{H}(8 \mathrm{~A})-\mathrm{C}(8)-\mathrm{H}(8 \mathrm{C})$ & 109.5 \\
\hline $\mathrm{H}(8 \mathrm{~B})-\mathrm{C}(8)-\mathrm{H}(8 \mathrm{C})$ & 109.5 \\
\hline $\mathrm{C}(3)-\mathrm{C}(9)-\mathrm{H}(9 \mathrm{~A})$ & 109.5 \\
\hline $\mathrm{C}(3)-\mathrm{C}(9)-\mathrm{H}(9 \mathrm{~B})$ & 109.5 \\
\hline $\mathrm{H}(9 \mathrm{~A})-\mathrm{C}(9)-\mathrm{H}(9 \mathrm{~B})$ & 109.5 \\
\hline
\end{tabular}




\begin{tabular}{|c|c|}
\hline$C(3)-C(9)-H(9 C)$ & 109.5 \\
\hline $\mathrm{H}(9 \mathrm{~A})-\mathrm{C}(9)-\mathrm{H}(9 \mathrm{C})$ & 109.5 \\
\hline $\mathrm{H}(9 \mathrm{~B})-\mathrm{C}(9)-\mathrm{H}(9 \mathrm{C})$ & 109.5 \\
\hline $\mathrm{N}(2)-\mathrm{C}(10)-\mathrm{N}(1)$ & 107.1(4) \\
\hline $\mathrm{N}(2)-\mathrm{C}(10)-\mathrm{Ru}(1)$ & $121.1(3)$ \\
\hline $\mathrm{N}(1)-\mathrm{C}(10)-\mathrm{Ru}(1)$ & $131.0(3)$ \\
\hline$N(1)-C(11)-C(12)$ & $102.0(4)$ \\
\hline $\mathrm{N}(1)-\mathrm{C}(11)-\mathrm{H}(11 \mathrm{~A})$ & 111.4 \\
\hline $\mathrm{C}(12)-\mathrm{C}(11)-\mathrm{H}(11 \mathrm{~A})$ & 111.4 \\
\hline $\mathrm{N}(1)-\mathrm{C}(11)-\mathrm{H}(11 \mathrm{~B})$ & 111.4 \\
\hline $\mathrm{C}(12)-\mathrm{C}(11)-\mathrm{H}(11 \mathrm{~B})$ & 111.4 \\
\hline $\mathrm{H}(11 \mathrm{~A})-\mathrm{C}(11)-\mathrm{H}(11 \mathrm{~B})$ & 109.2 \\
\hline $\mathrm{N}(2)-\mathrm{C}(12)-\mathrm{C}(11)$ & 101.7(4) \\
\hline $\mathrm{N}(2)-\mathrm{C}(12)-\mathrm{H}(12 \mathrm{~A})$ & 111.4 \\
\hline $\mathrm{C}(11)-\mathrm{C}(12)-\mathrm{H}(12 \mathrm{~A})$ & 111.4 \\
\hline $\mathrm{N}(2)-\mathrm{C}(12)-\mathrm{H}(12 \mathrm{~B})$ & 111.4 \\
\hline $\mathrm{C}(11)-\mathrm{C}(12)-\mathrm{H}(12 \mathrm{~B})$ & 111.4 \\
\hline $\mathrm{H}(12 \mathrm{~A})-\mathrm{C}(12)-\mathrm{H}(12 \mathrm{~B})$ & 109.3 \\
\hline$C(18)-C(13)-C(14)$ & 121.1(5) \\
\hline $\mathrm{C}(18)-\mathrm{C}(13)-\mathrm{N}(2)$ & $118.3(4)$ \\
\hline$C(14)-C(13)-N(2)$ & $120.0(5)$ \\
\hline$C(15)-C(14)-C(13)$ & $117.7(5)$ \\
\hline$C(15)-C(14)-C(20)$ & $120.3(5)$ \\
\hline$C(13)-C(14)-C(20)$ & $121.9(5)$ \\
\hline$C(16)-C(15)-C(14)$ & $122.2(5)$ \\
\hline$C(16)-C(15)-H(15)$ & 118.9 \\
\hline$C(14)-C(15)-H(15)$ & 118.9 \\
\hline$C(15)-C(16)-C(17)$ & $118.6(5)$ \\
\hline$C(15)-C(16)-C(21)$ & $120.6(5)$ \\
\hline$C(17)-C(16)-C(21)$ & $120.8(5)$ \\
\hline$C(16)-C(17)-C(18)$ & $122.0(5)$ \\
\hline $\mathrm{C}(16)-\mathrm{C}(17)-\mathrm{H}(17)$ & 119.0 \\
\hline $\mathrm{C}(18)-\mathrm{C}(17)-\mathrm{H}(17)$ & 119.0 \\
\hline$C(17)-C(18)-C(13)$ & 118.1(5) \\
\hline$C(17)-C(18)-C(19)$ & $120.3(5)$ \\
\hline$C(13)-C(18)-C(19)$ & $121.5(5)$ \\
\hline
\end{tabular}




\begin{tabular}{|c|c|}
\hline $\mathrm{C}(18)-\mathrm{C}(19)-\mathrm{H}(19 \mathrm{~A})$ & 109.5 \\
\hline $\mathrm{C}(18)-\mathrm{C}(19)-\mathrm{H}(19 \mathrm{~B})$ & 109.5 \\
\hline $\mathrm{H}(19 \mathrm{~A})-\mathrm{C}(19)-\mathrm{H}(19 \mathrm{~B})$ & 109.5 \\
\hline $\mathrm{C}(18)-\mathrm{C}(19)-\mathrm{H}(19 \mathrm{C})$ & 109.5 \\
\hline $\mathrm{H}(19 \mathrm{~A})-\mathrm{C}(19)-\mathrm{H}(19 \mathrm{C})$ & 109.5 \\
\hline $\mathrm{H}(19 \mathrm{~B})-\mathrm{C}(19)-\mathrm{H}(19 \mathrm{C})$ & 109.5 \\
\hline $\mathrm{C}(14)-\mathrm{C}(20)-\mathrm{H}(20 \mathrm{~A})$ & 109.5 \\
\hline $\mathrm{C}(14)-\mathrm{C}(20)-\mathrm{H}(20 \mathrm{~B})$ & 109.5 \\
\hline $\mathrm{H}(20 \mathrm{~A})-\mathrm{C}(20)-\mathrm{H}(20 \mathrm{~B})$ & 109.5 \\
\hline $\mathrm{C}(14)-\mathrm{C}(20)-\mathrm{H}(20 \mathrm{C})$ & 109.5 \\
\hline $\mathrm{H}(20 \mathrm{~A})-\mathrm{C}(20)-\mathrm{H}(20 \mathrm{C})$ & 109.5 \\
\hline $\mathrm{H}(20 \mathrm{~B})-\mathrm{C}(20)-\mathrm{H}(20 \mathrm{C})$ & 109.5 \\
\hline$C(16)-C(21)-H(21 A)$ & 109.5 \\
\hline $\mathrm{C}(16)-\mathrm{C}(21)-\mathrm{H}(21 \mathrm{~B})$ & 109.5 \\
\hline $\mathrm{H}(21 \mathrm{~A})-\mathrm{C}(21)-\mathrm{H}(21 \mathrm{~B})$ & 109.5 \\
\hline$C(16)-C(21)-H(21 C)$ & 109.5 \\
\hline $\mathrm{H}(21 \mathrm{~A})-\mathrm{C}(21)-\mathrm{H}(21 \mathrm{C})$ & 109.5 \\
\hline $\mathrm{H}(21 \mathrm{~B})-\mathrm{C}(21)-\mathrm{H}(21 \mathrm{C})$ & 109.5 \\
\hline$C(23)-C(22)-R u(1)$ & $139.7(4)$ \\
\hline $\mathrm{C}(23)-\mathrm{C}(22)-\mathrm{H}(22)$ & 110.2 \\
\hline $\mathrm{Ru}(1)-\mathrm{C}(22)-\mathrm{H}(22)$ & 110.2 \\
\hline$C(28)-C(23)-C(24)$ & $116.6(5)$ \\
\hline$C(28)-C(23)-C(22)$ & $117.0(5)$ \\
\hline$C(24)-C(23)-C(22)$ & $126.4(4)$ \\
\hline$C(25)-C(24)-C(23)$ & $120.6(5)$ \\
\hline $\mathrm{C}(25)-\mathrm{C}(24)-\mathrm{H}(24)$ & 119.7 \\
\hline $\mathrm{C}(23)-\mathrm{C}(24)-\mathrm{H}(24)$ & 119.7 \\
\hline$C(24)-C(25)-C(26)$ & $121.9(5)$ \\
\hline $\mathrm{C}(24)-\mathrm{C}(25)-\mathrm{H}(25)$ & 119.0 \\
\hline$C(26)-C(25)-H(25)$ & 119.0 \\
\hline $\mathrm{O}(1)-\mathrm{C}(26)-\mathrm{C}(27)$ & $124.1(5)$ \\
\hline $\mathrm{O}(1)-\mathrm{C}(26)-\mathrm{C}(25)$ & 117.1(5) \\
\hline$C(27)-C(26)-C(25)$ & $118.8(5)$ \\
\hline$C(26)-C(27)-C(28)$ & $119.0(5)$ \\
\hline $\mathrm{C}(26)-\mathrm{C}(27)-\mathrm{H}(27)$ & 120.5 \\
\hline $\mathrm{C}(28)-\mathrm{C}(27)-\mathrm{H}(27)$ & 120.5 \\
\hline
\end{tabular}




\begin{tabular}{|c|c|}
\hline$C(23)-C(28)-C(27)$ & $122.9(5)$ \\
\hline $\mathrm{C}(23)-\mathrm{C}(28)-\mathrm{H}(28)$ & 118.5 \\
\hline $\mathrm{C}(27)-\mathrm{C}(28)-\mathrm{H}(28)$ & 118.5 \\
\hline $\mathrm{O}(1)-\mathrm{C}(29)-\mathrm{C}(30)$ & $107.9(4)$ \\
\hline $\mathrm{O}(1)-\mathrm{C}(29)-\mathrm{H}(29 \mathrm{~A})$ & 110.1 \\
\hline $\mathrm{C}(30)-\mathrm{C}(29)-\mathrm{H}(29 \mathrm{~A})$ & 110.1 \\
\hline $\mathrm{O}(1)-\mathrm{C}(29)-\mathrm{H}(29 \mathrm{~B})$ & 110.1 \\
\hline $\mathrm{C}(30)-\mathrm{C}(29)-\mathrm{H}(29 \mathrm{~B})$ & 110.1 \\
\hline $\mathrm{H}(29 \mathrm{~A})-\mathrm{C}(29)-\mathrm{H}(29 \mathrm{~B})$ & 108.4 \\
\hline $\mathrm{C}(31)-\mathrm{C}(30)-\mathrm{C}(29)$ & $115.7(4)$ \\
\hline $\mathrm{C}(31)-\mathrm{C}(30)-\mathrm{H}(30 \mathrm{~A})$ & 108.4 \\
\hline $\mathrm{C}(29)-\mathrm{C}(30)-\mathrm{H}(30 \mathrm{~A})$ & 108.4 \\
\hline $\mathrm{C}(31)-\mathrm{C}(30)-\mathrm{H}(30 \mathrm{~B})$ & 108.4 \\
\hline $\mathrm{C}(29)-\mathrm{C}(30)-\mathrm{H}(30 \mathrm{~B})$ & 108.4 \\
\hline $\mathrm{H}(30 \mathrm{~A})-\mathrm{C}(30)-\mathrm{H}(30 \mathrm{~B})$ & 107.4 \\
\hline$C(30)-C(31)-C(32)$ & $116.9(5)$ \\
\hline $\mathrm{C}(30)-\mathrm{C}(31)-\mathrm{H}(31 \mathrm{~A})$ & 108.1 \\
\hline $\mathrm{C}(32)-\mathrm{C}(31)-\mathrm{H}(31 \mathrm{~A})$ & 108.1 \\
\hline $\mathrm{C}(30)-\mathrm{C}(31)-\mathrm{H}(31 \mathrm{~B})$ & 108.1 \\
\hline $\mathrm{C}(32)-\mathrm{C}(31)-\mathrm{H}(31 \mathrm{~B})$ & 108.1 \\
\hline $\mathrm{H}(31 \mathrm{~A})-\mathrm{C}(31)-\mathrm{H}(31 \mathrm{~B})$ & 107.3 \\
\hline$C(33)-C(32)-C(31)$ & $118.4(4)$ \\
\hline $\mathrm{C}(33)-\mathrm{C}(32)-\mathrm{H}(32 \mathrm{~A})$ & 107.7 \\
\hline $\mathrm{C}(31)-\mathrm{C}(32)-\mathrm{H}(32 \mathrm{~A})$ & 107.7 \\
\hline $\mathrm{C}(33)-\mathrm{C}(32)-\mathrm{H}(32 \mathrm{~B})$ & 107.7 \\
\hline $\mathrm{C}(31)-\mathrm{C}(32)-\mathrm{H}(32 \mathrm{~B})$ & 107.7 \\
\hline $\mathrm{H}(32 \mathrm{~A})-\mathrm{C}(32)-\mathrm{H}(32 \mathrm{~B})$ & 107.1 \\
\hline$C(34)-C(33)-C(32)$ & 113.1(4) \\
\hline $\mathrm{C}(34)-\mathrm{C}(33)-\mathrm{H}(33 \mathrm{~A})$ & 109.0 \\
\hline $\mathrm{C}(32)-\mathrm{C}(33)-\mathrm{H}(33 \mathrm{~A})$ & 109.0 \\
\hline $\mathrm{C}(34)-\mathrm{C}(33)-\mathrm{H}(33 \mathrm{~B})$ & 109.0 \\
\hline $\mathrm{C}(32)-\mathrm{C}(33)-\mathrm{H}(33 \mathrm{~B})$ & 109.0 \\
\hline $\mathrm{H}(33 \mathrm{~A})-\mathrm{C}(33)-\mathrm{H}(33 \mathrm{~B})$ & 107.8 \\
\hline $\mathrm{O}(2)-\mathrm{C}(34)-\mathrm{C}(33)$ & $107.9(4)$ \\
\hline $\mathrm{O}(2)-\mathrm{C}(34)-\mathrm{H}(34 \mathrm{~A})$ & 110.1 \\
\hline $\mathrm{C}(33)-\mathrm{C}(34)-\mathrm{H}(34 \mathrm{~A})$ & 110.1 \\
\hline
\end{tabular}




\begin{tabular}{|c|c|}
\hline $\mathrm{O}(2)-\mathrm{C}(34)-\mathrm{H}(34 \mathrm{~B})$ & 110.1 \\
\hline $\mathrm{C}(33)-\mathrm{C}(34)-\mathrm{H}(34 \mathrm{~B})$ & 110.1 \\
\hline $\mathrm{H}(34 \mathrm{~A})-\mathrm{C}(34)-\mathrm{H}(34 \mathrm{~B})$ & 108.4 \\
\hline$C(40)-C(35)-C(36)$ & $110.1(4)$ \\
\hline $\mathrm{C}(40)-\mathrm{C}(35)-\mathrm{P}(1)$ & $118.1(3)$ \\
\hline$C(36)-C(35)-P(1)$ & $111.5(3)$ \\
\hline $\mathrm{C}(40)-\mathrm{C}(35)-\mathrm{H}(35)$ & 105.3 \\
\hline $\mathrm{C}(36)-\mathrm{C}(35)-\mathrm{H}(35)$ & 105.3 \\
\hline $\mathrm{P}(1)-\mathrm{C}(35)-\mathrm{H}(35)$ & 105.3 \\
\hline$C(37)-C(36)-C(35)$ & $111.5(4)$ \\
\hline $\mathrm{C}(37)-\mathrm{C}(36)-\mathrm{H}(36 \mathrm{~A})$ & 109.3 \\
\hline $\mathrm{C}(35)-\mathrm{C}(36)-\mathrm{H}(36 \mathrm{~A})$ & 109.3 \\
\hline $\mathrm{C}(37)-\mathrm{C}(36)-\mathrm{H}(36 \mathrm{~B})$ & 109.3 \\
\hline $\mathrm{C}(35)-\mathrm{C}(36)-\mathrm{H}(36 \mathrm{~B})$ & 109.3 \\
\hline $\mathrm{H}(36 \mathrm{~A})-\mathrm{C}(36)-\mathrm{H}(36 \mathrm{~B})$ & 108.0 \\
\hline$C(38)-C(37)-C(36)$ & $109.5(4)$ \\
\hline $\mathrm{C}(38)-\mathrm{C}(37)-\mathrm{H}(37 \mathrm{~A})$ & 109.8 \\
\hline $\mathrm{C}(36)-\mathrm{C}(37)-\mathrm{H}(37 \mathrm{~A})$ & 109.8 \\
\hline $\mathrm{C}(38)-\mathrm{C}(37)-\mathrm{H}(37 \mathrm{~B})$ & 109.8 \\
\hline $\mathrm{C}(36)-\mathrm{C}(37)-\mathrm{H}(37 \mathrm{~B})$ & 109.8 \\
\hline $\mathrm{H}(37 \mathrm{~A})-\mathrm{C}(37)-\mathrm{H}(37 \mathrm{~B})$ & 108.2 \\
\hline$C(39)-C(38)-C(37)$ & 112.1(4) \\
\hline $\mathrm{C}(39)-\mathrm{C}(38)-\mathrm{H}(38 \mathrm{~A})$ & 109.2 \\
\hline $\mathrm{C}(37)-\mathrm{C}(38)-\mathrm{H}(38 \mathrm{~A})$ & 109.2 \\
\hline $\mathrm{C}(39)-\mathrm{C}(38)-\mathrm{H}(38 \mathrm{~B})$ & 109.2 \\
\hline $\mathrm{C}(37)-\mathrm{C}(38)-\mathrm{H}(38 \mathrm{~B})$ & 109.2 \\
\hline $\mathrm{H}(38 \mathrm{~A})-\mathrm{C}(38)-\mathrm{H}(38 \mathrm{~B})$ & 107.9 \\
\hline$C(38)-C(39)-C(40)$ & $112.0(4)$ \\
\hline $\mathrm{C}(38)-\mathrm{C}(39)-\mathrm{H}(39 \mathrm{~A})$ & 109.2 \\
\hline $\mathrm{C}(40)-\mathrm{C}(39)-\mathrm{H}(39 \mathrm{~A})$ & 109.2 \\
\hline $\mathrm{C}(38)-\mathrm{C}(39)-\mathrm{H}(39 \mathrm{~B})$ & 109.2 \\
\hline $\mathrm{C}(40)-\mathrm{C}(39)-\mathrm{H}(39 \mathrm{~B})$ & 109.2 \\
\hline $\mathrm{H}(39 \mathrm{~A})-\mathrm{C}(39)-\mathrm{H}(39 \mathrm{~B})$ & 107.9 \\
\hline$C(39)-C(40)-C(35)$ & $110.6(4)$ \\
\hline $\mathrm{C}(39)-\mathrm{C}(40)-\mathrm{H}(40 \mathrm{~A})$ & 109.5 \\
\hline $\mathrm{C}(35)-\mathrm{C}(40)-\mathrm{H}(40 \mathrm{~A})$ & 109.5 \\
\hline
\end{tabular}




$\begin{array}{ll}\mathrm{C}(39)-\mathrm{C}(40)-\mathrm{H}(40 \mathrm{~B}) & 109.5 \\ \mathrm{C}(35)-\mathrm{C}(40)-\mathrm{H}(40 \mathrm{~B}) & 109.5 \\ \mathrm{H}(40 \mathrm{~A})-\mathrm{C}(40)-\mathrm{H}(40 \mathrm{~B}) & 108.1 \\ \mathrm{C}(46)-\mathrm{C}(41)-\mathrm{C}(42) & 109.1(4) \\ \mathrm{C}(46)-\mathrm{C}(41)-\mathrm{P}(1) & 116.8(3) \\ \mathrm{C}(42)-\mathrm{C}(41)-\mathrm{P}(1) & 116.2(3) \\ \mathrm{C}(46)-\mathrm{C}(41)-\mathrm{H}(41) & 104.3 \\ \mathrm{C}(42)-\mathrm{C}(41)-\mathrm{H}(41) & 104.3 \\ \mathrm{P}(1)-\mathrm{C}(41)-\mathrm{H}(41) & 104.3 \\ \mathrm{C}(43)-\mathrm{C}(42)-\mathrm{C}(41) & 111.0(4) \\ \mathrm{C}(43)-\mathrm{C}(42)-\mathrm{H}(42 \mathrm{~A}) & 109.4 \\ \mathrm{C}(41)-\mathrm{C}(42)-\mathrm{H}(42 \mathrm{~A}) & 109.4 \\ \mathrm{C}(43)-\mathrm{C}(42)-\mathrm{H}(42 \mathrm{~B}) & 109.4 \\ \mathrm{C}(41)-\mathrm{C}(42)-\mathrm{H}(42 \mathrm{~B}) & 109.4 \\ \mathrm{H}(42 \mathrm{~A})-\mathrm{C}(42)-\mathrm{H}(42 \mathrm{~B}) & 108.0 \\ \mathrm{C}(42)-\mathrm{C}(43)-\mathrm{C}(44) & 111.5(4) \\ \mathrm{C}(42)-\mathrm{C}(43)-\mathrm{H}(43 \mathrm{~A}) & 109.3 \\ \mathrm{C}(44)-\mathrm{C}(43)-\mathrm{H}(43 \mathrm{~A}) & 109.3 \\ \mathrm{C}(42)-\mathrm{C}(43)-\mathrm{H}(43 \mathrm{~B}) & 109.3 \\ \mathrm{C}(44)-\mathrm{C}(43)-\mathrm{H}(43 \mathrm{~B}) & 109.3 \\ \mathrm{H}(43 \mathrm{~A})-\mathrm{C}(43)-\mathrm{H}(43 \mathrm{~B}) & 108.0 \\ \mathrm{C}(45)-\mathrm{C}(44)-\mathrm{C}(43) & 110.0(4) \\ \mathrm{C}(45)-\mathrm{C}(44)-\mathrm{H}(44 \mathrm{~A}) & 109.7 \\ \mathrm{C}(43)-\mathrm{C}(44)-\mathrm{H}(44 \mathrm{~A}) & 109.7 \\ \mathrm{C}(45)-\mathrm{C}(44)-\mathrm{H}(44 \mathrm{~B}) & 109.7 \\ \mathrm{C}(43)-\mathrm{C}(44)-\mathrm{H}(44 \mathrm{~B}) & 109.7 \\ \mathrm{H}(44 \mathrm{~A})-\mathrm{C}(44)-\mathrm{H}(44 \mathrm{~B}) & 108.2 \\ \mathrm{C}(44)-\mathrm{C}(45)-\mathrm{C}(46) & 112.3(4) \\ \mathrm{C}(44)-\mathrm{C}(45)-\mathrm{H}(45 \mathrm{~A}) & 109.1 \\ \mathrm{C}(46)-\mathrm{C}(45)-\mathrm{H}(45 \mathrm{~A}) & 109.1 \\ \mathrm{C}(44)-\mathrm{C}(45)-\mathrm{H}(45 \mathrm{~B}) & 109.1 \\ \mathrm{C}(46)-\mathrm{C}(45)-\mathrm{H}(45 \mathrm{~B}) & 109.1 \\ \mathrm{C}(45 \mathrm{~A})-\mathrm{C}(45)-\mathrm{H}(45 \mathrm{~B}) & 107.9 \\ \mathrm{C}(46)-\mathrm{C}(41) & 109.4(4) \\ & 109.8 \\ & 109.8 \\ & \end{array}$




$\begin{array}{ll}\mathrm{C}(45)-\mathrm{C}(46)-\mathrm{H}(46 \mathrm{~B}) & 109.8 \\ \mathrm{C}(41)-\mathrm{C}(46)-\mathrm{H}(46 \mathrm{~B}) & 109.8 \\ \mathrm{H}(46 \mathrm{~A})-\mathrm{C}(46)-\mathrm{H}(46 \mathrm{~B}) & 108.3 \\ \mathrm{C}(52)-\mathrm{C}(47)-\mathrm{C}(48) & 111.5(4) \\ \mathrm{C}(52)-\mathrm{C}(47)-\mathrm{P}(1) & 112.3(3) \\ \mathrm{C}(48)-\mathrm{C}(47)-\mathrm{P}(1) & 111.5(3) \\ \mathrm{C}(52)-\mathrm{C}(47)-\mathrm{H}(47) & 107.1 \\ \mathrm{C}(48)-\mathrm{C}(47)-\mathrm{H}(47) & 107.1 \\ \mathrm{P}(1)-\mathrm{C}(47)-\mathrm{H}(47) & 107.1 \\ \mathrm{C}(49)-\mathrm{C}(48)-\mathrm{C}(47) & 109.8(4) \\ \mathrm{C}(49)-\mathrm{C}(48)-\mathrm{H}(48 \mathrm{~A}) & 109.7 \\ \mathrm{C}(47)-\mathrm{C}(48)-\mathrm{H}(48 \mathrm{~A}) & 109.7 \\ \mathrm{C}(49)-\mathrm{C}(48)-\mathrm{H}(48 \mathrm{~B}) & 109.7 \\ \mathrm{C}(47)-\mathrm{C}(48)-\mathrm{H}(48 \mathrm{~B}) & 109.7 \\ \mathrm{H}(48 \mathrm{~A})-\mathrm{C}(48)-\mathrm{H}(48 \mathrm{~B}) & 108.2 \\ \mathrm{C}(48)-\mathrm{C}(49)-\mathrm{C}(50) & 111.6(4) \\ \mathrm{C}(48)-\mathrm{C}(49)-\mathrm{H}(49 \mathrm{~A}) & 109.3 \\ \mathrm{C}(50)-\mathrm{C}(49)-\mathrm{H}(49 \mathrm{~A}) & 109.3 \\ \mathrm{C}(48)-\mathrm{C}(49)-\mathrm{H}(49 \mathrm{~B}) & 109.3 \\ \mathrm{C}(50)-\mathrm{C}(49)-\mathrm{H}(49 \mathrm{~B}) & 109.3 \\ \mathrm{H}(49 \mathrm{~A})-\mathrm{C}(49)-\mathrm{H}(49 \mathrm{~B}) & 108.0 \\ \mathrm{C}(49)-\mathrm{C}(50)-\mathrm{C}(51) & 109.9(4) \\ \mathrm{C}(49)-\mathrm{C}(50)-\mathrm{H}(50 \mathrm{~A}) & 109.7 \\ \mathrm{C}(51)-\mathrm{C}(50)-\mathrm{H}(50 \mathrm{~A}) & 109.7 \\ \mathrm{C}(49)-\mathrm{C}(50)-\mathrm{H}(50 \mathrm{~B}) & 109.7 \\ \mathrm{C}(51)-\mathrm{C}(50)-\mathrm{H}(50 \mathrm{~B}) & 109.7 \\ \mathrm{H}(50 \mathrm{~A})-\mathrm{C}(50)-\mathrm{H}(50 \mathrm{~B}) & 108.2 \\ \mathrm{C}(50)-\mathrm{C}(51)-\mathrm{C}(52) & 111.2(4) \\ \mathrm{C}(50)-\mathrm{C}(51)-\mathrm{H}(51 \mathrm{~A}) & 109.4 \\ \mathrm{C}(52)-\mathrm{C}(51)-\mathrm{H}(51 \mathrm{~A}) & 109.4 \\ \mathrm{C}(50)-\mathrm{C}(51)-\mathrm{H}(51 \mathrm{~B}) & 109.4 \\ \mathrm{C}(52)-\mathrm{C}(51)-\mathrm{H}(51 \mathrm{~B}) & 109.4 \\ \mathrm{H}(51 \mathrm{~A})-\mathrm{C}(51)-\mathrm{H}(51 \mathrm{~B}) & 108.0 \\ \mathrm{C}(51)-\mathrm{C}(52)-\mathrm{H}(52 \mathrm{~A}) & 109.2 \\ \mathrm{C}(47)-\mathrm{C}(52)-\mathrm{H}(52 \mathrm{~A}) & 109.2 \\ & \end{array}$




\begin{tabular}{ll}
$\mathrm{C}(51)-\mathrm{C}(52)-\mathrm{H}(52 \mathrm{~B})$ & 109.2 \\
$\mathrm{C}(47)-\mathrm{C}(52)-\mathrm{H}(52 \mathrm{~B})$ & 109.2 \\
$\mathrm{H}(52 \mathrm{~A})-\mathrm{C}(52)-\mathrm{H}(52 \mathrm{~B})$ & 107.9 \\
$\mathrm{C}(10)-\mathrm{N}(1)-\mathrm{C}(2)$ & $127.8(4)$ \\
$\mathrm{C}(10)-\mathrm{N}(1)-\mathrm{C}(11)$ & $112.9(4)$ \\
$\mathrm{C}(2)-\mathrm{N}(1)-\mathrm{C}(11)$ & $117.2(4)$ \\
$\mathrm{C}(10)-\mathrm{N}(2)-\mathrm{C}(13)$ & $126.5(4)$ \\
$\mathrm{C}(10)-\mathrm{N}(2)-\mathrm{C}(12)$ & $113.8(4)$ \\
$\mathrm{C}(13)-\mathrm{N}(2)-\mathrm{C}(12)$ & $119.6(4)$ \\
$\mathrm{C}(26)-\mathrm{O}(1)-\mathrm{C}(29)$ & $119.5(4)$ \\
$\mathrm{C}(5)-\mathrm{O}(2)-\mathrm{C}(34)$ & $117.9(4)$ \\
$\mathrm{C}(41)-\mathrm{P}(1)-\mathrm{C}(35)$ & $109.5(2)$ \\
$\mathrm{C}(41)-\mathrm{P}(1)-\mathrm{C}(47)$ & $101.4(2)$ \\
$\mathrm{C}(35)-\mathrm{P}(1)-\mathrm{C}(47)$ & $104.6(2)$ \\
$\mathrm{C}(41)-\mathrm{P}(1)-\mathrm{Ru}(1)$ & $110.64(16)$ \\
$\mathrm{C}(35)-\mathrm{P}(1)-\mathrm{Ru}(1)$ & $112.21(16)$ \\
$\mathrm{C}(47)-\mathrm{P}(1)-\mathrm{Ru}(1)$ & $117.62(16)$ \\
$\mathrm{C}(22)-\mathrm{Ru}(1)-\mathrm{C}(10)$ & $100.96(19)$ \\
$\mathrm{C}(22)-\mathrm{Ru}(1)-\mathrm{Cl}(1)$ & $101.27(17)$ \\
$\mathrm{C}(10)-\mathrm{Ru}(1)-\mathrm{Cl}(1)$ & $92.25(14)$ \\
$\mathrm{C}(22)-\mathrm{Ru}(1)-\mathrm{Cl}(2)$ & $85.32(16)$ \\
$\mathrm{C}(10)-\mathrm{Ru}(1)-\mathrm{Cl}(2)$ & $86.45(14)$ \\
$\mathrm{Cl}(1)-\mathrm{Ru}(1)-\mathrm{Cl}(2)$ & $173.42(5)$ \\
$\mathrm{C}(22)-\mathrm{Ru}(1)-\mathrm{P}(1)$ & $98.75(15)$ \\
$\mathrm{C}(10)-\mathrm{Ru}(1)-\mathrm{P}(1)$ & $159.86(13)$ \\
$\mathrm{Cl}(1)-\mathrm{Ru}(1)-\mathrm{P}(1)$ & $87.88(5)$ \\
$\mathrm{Cl}(2)-\mathrm{Ru}(1)-\mathrm{P}(1)$ & $91.12(5)$ \\
\hline
\end{tabular}


Table S4. Anisotropic displacement parameters $\left(\AA^{2} \times 10^{3}\right)$ for CB6. The anisotropic displacement factor exponent takes the form: $-2 \mathrm{p}^{2}\left[\mathrm{~h}^{2} \mathrm{a}^{* 2} \mathrm{U}^{11}+\ldots+2 \mathrm{~h} \mathrm{k} \mathrm{a}^{*} \mathrm{~b}^{*} \mathrm{U}^{12}\right]$

\begin{tabular}{|c|c|c|c|c|c|c|}
\hline & $\mathrm{U}^{11}$ & $\mathrm{U}^{22}$ & $\mathrm{U}^{33}$ & $\mathrm{U}^{23}$ & $\mathrm{U}^{13}$ & $\mathrm{U}^{12}$ \\
\hline$C(2)$ & $10(3)$ & $19(3)$ & $27(3)$ & $5(3)$ & $7(2)$ & $1(2)$ \\
\hline$C(3)$ & $8(3)$ & $32(3)$ & $17(3)$ & $9(3)$ & $1(2)$ & $5(3)$ \\
\hline$C(4)$ & $19(3)$ & $17(3)$ & $35(4)$ & $3(3)$ & $5(3)$ & $0(3)$ \\
\hline$C(5)$ & $15(3)$ & $21(3)$ & $34(4)$ & $9(3)$ & $-3(3)$ & $6(2)$ \\
\hline$C(6)$ & $15(3)$ & $29(3)$ & $22(3)$ & $2(3)$ & $0(2)$ & $6(3)$ \\
\hline$C(7)$ & $12(3)$ & $20(3)$ & $28(3)$ & $5(3)$ & $9(2)$ & $4(2)$ \\
\hline$C(8)$ & $27(3)$ & $23(3)$ & $36(4)$ & $4(3)$ & $1(3)$ & $8(3)$ \\
\hline$C(9)$ & $19(3)$ & $25(3)$ & $37(4)$ & $-4(3)$ & $0(3)$ & $4(3)$ \\
\hline$C(10)$ & $23(3)$ & $16(3)$ & 21(3) & $-2(2)$ & $1(2)$ & $7(2)$ \\
\hline$C(11)$ & $18(3)$ & $31(3)$ & $29(3)$ & $13(3)$ & $6(3)$ & $-1(3)$ \\
\hline $\mathrm{C}(12)$ & $25(3)$ & $39(4)$ & $45(4)$ & $28(3)$ & $-1(3)$ & $1(3)$ \\
\hline$C(13)$ & $21(3)$ & $17(3)$ & $25(3)$ & $8(3)$ & $-2(3)$ & $3(2)$ \\
\hline$C(14)$ & $27(3)$ & $18(3)$ & $25(3)$ & $5(3)$ & $5(3)$ & $-3(3)$ \\
\hline$C(15)$ & $17(3)$ & $15(3)$ & $40(4)$ & $10(3)$ & $10(3)$ & $9(2)$ \\
\hline$C(16)$ & $25(3)$ & $23(3)$ & $25(3)$ & $9(3)$ & $1(3)$ & 1(3) \\
\hline$C(17)$ & $27(3)$ & $26(3)$ & $20(3)$ & $0(3)$ & $3(3)$ & $0(3)$ \\
\hline C(18) & $22(3)$ & $24(3)$ & $22(3)$ & $10(3)$ & $9(3)$ & $5(3)$ \\
\hline$C(19)$ & $40(4)$ & $30(4)$ & $31(3)$ & $3(3)$ & $8(3)$ & $9(3)$ \\
\hline$C(20)$ & $26(3)$ & $26(3)$ & $31(3)$ & $6(3)$ & $4(3)$ & $6(3)$ \\
\hline$C(21)$ & $26(4)$ & $45(4)$ & $39(4)$ & $14(3)$ & $-2(3)$ & $11(3)$ \\
\hline$C(22)$ & $15(3)$ & $23(3)$ & $29(3)$ & $-5(3)$ & $1(2)$ & $-1(2)$ \\
\hline$C(23)$ & $15(3)$ & $27(3)$ & $23(3)$ & $3(3)$ & $9(2)$ & $4(3)$ \\
\hline$C(24)$ & $24(3)$ & $32(4)$ & $25(3)$ & $2(3)$ & $4(3)$ & $10(3)$ \\
\hline$C(25)$ & $26(3)$ & $27(3)$ & $30(4)$ & $-5(3)$ & $0(3)$ & $5(3)$ \\
\hline$C(26)$ & $24(3)$ & $23(3)$ & $27(3)$ & $0(3)$ & $-2(3)$ & $6(3)$ \\
\hline$C(27)$ & $27(3)$ & $31(4)$ & $31(4)$ & $11(3)$ & $1(3)$ & $9(3)$ \\
\hline$C(28)$ & $18(3)$ & $25(3)$ & $29(3)$ & $3(3)$ & $-4(3)$ & $-2(3)$ \\
\hline$C(29)$ & $30(4)$ & $37(4)$ & $30(4)$ & $9(3)$ & $-3(3)$ & $7(3)$ \\
\hline$C(30)$ & $36(4)$ & $24(3)$ & $40(4)$ & $4(3)$ & $-2(3)$ & $7(3)$ \\
\hline$C(31)$ & $43(4)$ & $20(3)$ & $36(4)$ & $1(3)$ & $-2(3)$ & $9(3)$ \\
\hline$C(32)$ & $37(4)$ & $31(4)$ & $34(4)$ & $5(3)$ & $-2(3)$ & $10(3)$ \\
\hline
\end{tabular}




\begin{tabular}{|c|c|c|c|c|c|c|}
\hline$C(33)$ & $28(3)$ & $18(3)$ & $33(4)$ & $4(3)$ & $-5(3)$ & $4(3)$ \\
\hline$C(34)$ & $19(3)$ & $24(3)$ & $30(3)$ & $0(3)$ & $0(3)$ & $6(3)$ \\
\hline$C(35)$ & $9(3)$ & $21(3)$ & $21(3)$ & $-2(2)$ & $0(2)$ & $5(2)$ \\
\hline$C(36)$ & $21(3)$ & $29(3)$ & $19(3)$ & $6(3)$ & $1(2)$ & $3(3)$ \\
\hline$C(37)$ & $43(4)$ & $27(3)$ & $20(3)$ & $-3(3)$ & $-8(3)$ & $11(3)$ \\
\hline C(38) & $43(4)$ & $35(4)$ & $27(3)$ & $-3(3)$ & $-1(3)$ & $25(3)$ \\
\hline C(39) & $25(3)$ & $42(4)$ & $30(4)$ & $2(3)$ & $6(3)$ & $15(3)$ \\
\hline$C(40)$ & $18(3)$ & $30(3)$ & $27(3)$ & $3(3)$ & $7(3)$ & $5(3)$ \\
\hline$C(41)$ & $20(3)$ & $25(3)$ & $18(3)$ & $1(2)$ & $-2(2)$ & $2(2)$ \\
\hline$C(42)$ & $20(3)$ & $23(3)$ & $26(3)$ & $7(3)$ & $2(3)$ & $4(2)$ \\
\hline$C(43)$ & $30(4)$ & $27(3)$ & $28(3)$ & 1(3) & $-5(3)$ & $-3(3)$ \\
\hline$C(44)$ & $27(3)$ & $41(4)$ & $22(3)$ & $-5(3)$ & $-1(3)$ & $3(3)$ \\
\hline$C(45)$ & $19(3)$ & $36(4)$ & $36(4)$ & $-7(3)$ & $-6(3)$ & $4(3)$ \\
\hline$C(46)$ & $19(3)$ & $38(4)$ & $26(3)$ & $9(3)$ & $1(3)$ & $5(3)$ \\
\hline$C(47)$ & $8(3)$ & $23(3)$ & $26(3)$ & $7(3)$ & $1(2)$ & $2(2)$ \\
\hline$C(48)$ & $18(3)$ & 21(3) & $25(3)$ & 1(3) & $2(2)$ & $-2(2)$ \\
\hline$C(49)$ & $27(3)$ & $26(3)$ & $24(3)$ & $7(3)$ & $2(3)$ & $7(3)$ \\
\hline$C(50)$ & $27(3)$ & $32(4)$ & $37(4)$ & $12(3)$ & $14(3)$ & $5(3)$ \\
\hline$C(51)$ & $24(3)$ & $24(3)$ & $34(4)$ & $-6(3)$ & $5(3)$ & $-2(3)$ \\
\hline$C(52)$ & $21(3)$ & $23(3)$ & $22(3)$ & $3(3)$ & $1(2)$ & $4(2)$ \\
\hline $\mathrm{N}(1)$ & $15(2)$ & $19(2)$ & $26(3)$ & $7(2)$ & $-1(2)$ & $6(2)$ \\
\hline $\mathrm{N}(2)$ & $13(2)$ & $23(3)$ & $29(3)$ & $8(2)$ & $2(2)$ & $-1(2)$ \\
\hline $\mathrm{O}(1)$ & $42(3)$ & $25(2)$ & $43(3)$ & $3(2)$ & $0(2)$ & $10(2)$ \\
\hline $\mathrm{O}(2)$ & $25(2)$ & $22(2)$ & $33(2)$ & $2(2)$ & $-6(2)$ & $7(2)$ \\
\hline $\mathrm{P}(1)$ & $14(1)$ & $22(1)$ & $21(1)$ & $2(1)$ & $1(1)$ & $4(1)$ \\
\hline $\mathrm{Cl}(1)$ & $20(1)$ & $28(1)$ & $25(1)$ & $-2(1)$ & $2(1)$ & $3(1)$ \\
\hline $\mathrm{Cl}(2)$ & $21(1)$ & $26(1)$ & $24(1)$ & $-1(1)$ & $1(1)$ & $1(1)$ \\
\hline $\mathrm{Ru}(1)$ & $14(1)$ & $19(1)$ & $22(1)$ & $2(1)$ & $2(1)$ & $3(1)$ \\
\hline
\end{tabular}


Table S5. Hydrogen coordinates $\left(\mathrm{x} 10^{4}\right)$ and isotropic displacement parameters $\left(\AA^{2} \mathrm{x} 10^{3}\right)$ for CB6.

\begin{tabular}{|c|c|c|c|c|}
\hline & $\mathrm{x}$ & $\mathrm{y}$ & $z$ & $\mathrm{U}(\mathrm{eq})$ \\
\hline $\mathrm{H}(4)$ & 11642 & -629 & 2104 & 29 \\
\hline $\mathrm{H}(6)$ & 12168 & 1116 & 4418 & 26 \\
\hline $\mathrm{H}(8 \mathrm{~A})$ & 11697 & 2826 & 4511 & 43 \\
\hline $\mathrm{H}(8 \mathrm{~B})$ & 10671 & 3058 & 3841 & 43 \\
\hline $\mathrm{H}(8 \mathrm{C})$ & 11877 & 3439 & 3585 & 43 \\
\hline $\mathrm{H}(9 \mathrm{~A})$ & 9884 & 588 & 951 & 41 \\
\hline $\mathrm{H}(9 \mathrm{~B})$ & 10939 & 50 & 733 & 41 \\
\hline $\mathrm{H}(9 \mathrm{C})$ & 10935 & 1285 & 646 & 41 \\
\hline $\mathrm{H}(11 \mathrm{~A})$ & 11766 & 2950 & 1056 & 31 \\
\hline $\mathrm{H}(11 \mathrm{~B})$ & 11971 & 3685 & 1961 & 31 \\
\hline $\mathrm{H}(12 \mathrm{~A})$ & 10756 & 4747 & 1414 & 44 \\
\hline $\mathrm{H}(12 \mathrm{~B})$ & 10817 & 4195 & 433 & 44 \\
\hline $\mathrm{H}(15)$ & 6877 & 5519 & 590 & 28 \\
\hline $\mathrm{H}(17)$ & 7315 & 3464 & -1381 & 29 \\
\hline $\mathrm{H}(19 \mathrm{~A})$ & 9812 & 2888 & -593 & 50 \\
\hline $\mathrm{H}(19 \mathrm{~B})$ & 8795 & 2494 & -1298 & 50 \\
\hline $\mathrm{H}(19 \mathrm{C})$ & 8929 & 1972 & -317 & 50 \\
\hline $\mathrm{H}(20 \mathrm{~A})$ & 8598 & 4719 & 2300 & 41 \\
\hline $\mathrm{H}(20 \mathrm{~B})$ & 7962 & 5683 & 1972 & 41 \\
\hline $\mathrm{H}(20 \mathrm{C})$ & 9200 & 5650 & 1756 & 41 \\
\hline $\mathrm{H}(21 \mathrm{~A})$ & 5356 & 4750 & -783 & 55 \\
\hline $\mathrm{H}(21 \mathrm{~B})$ & 5982 & 4591 & -1674 & 55 \\
\hline $\mathrm{H}(21 \mathrm{C})$ & 6163 & 5686 & -1133 & 55 \\
\hline $\mathrm{H}(22)$ & 8879 & 1400 & 3566 & 27 \\
\hline $\mathrm{H}(24)$ & 8540 & -520 & 1686 & 32 \\
\hline $\mathrm{H}(25)$ & 8937 & -2206 & 1893 & 33 \\
\hline $\mathrm{H}(27)$ & 9674 & -1653 & 4598 & 35 \\
\hline $\mathrm{H}(28)$ & 9287 & 65 & 4390 & 30 \\
\hline $\mathrm{H}(29 \mathrm{~A})$ & 9594 & -3697 & 4535 & 39 \\
\hline $\mathrm{H}(29 \mathrm{~B})$ & 10655 & -2878 & 4471 & 39 \\
\hline $\mathrm{H}(30 \mathrm{~A})$ & 11136 & -4600 & 4497 & 40 \\
\hline
\end{tabular}




\begin{tabular}{|c|c|c|c|c|}
\hline $\mathrm{H}(30 \mathrm{~B})$ & 10315 & -4899 & 3618 & 40 \\
\hline $\mathrm{H}(31 \mathrm{~A})$ & 11454 & -3615 & 2817 & 39 \\
\hline $\mathrm{H}(31 \mathrm{~B})$ & 11899 & -4709 & 3028 & 39 \\
\hline $\mathrm{H}(32 \mathrm{~A})$ & 13030 & -3926 & 4275 & 40 \\
\hline $\mathrm{H}(32 \mathrm{~B})$ & 13329 & -3471 & 3313 & 40 \\
\hline $\mathrm{H}(33 \mathrm{~A})$ & 12295 & -2418 & 4644 & 32 \\
\hline $\mathrm{H}(33 \mathrm{~B})$ & 13509 & -2124 & 4370 & 32 \\
\hline $\mathrm{H}(34 \mathrm{~A})$ & 12813 & -1633 & 2900 & 29 \\
\hline $\mathrm{H}(34 \mathrm{~B})$ & 11597 & -1912 & 3189 & 29 \\
\hline $\mathrm{H}(35)$ & 6350 & 2776 & 3710 & 20 \\
\hline $\mathrm{H}(36 \mathrm{~A})$ & 6607 & 3924 & 2485 & 28 \\
\hline $\mathrm{H}(36 \mathrm{~B})$ & 5325 & 3780 & 2203 & 28 \\
\hline $\mathrm{H}(37 \mathrm{~A})$ & 6325 & 4701 & 3898 & 36 \\
\hline $\mathrm{H}(37 \mathrm{~B})$ & 5757 & 5328 & 3088 & 36 \\
\hline $\mathrm{H}(38 \mathrm{~A})$ & 4563 & 4961 & 4261 & 41 \\
\hline $\mathrm{H}(38 \mathrm{~B})$ & 4050 & 4439 & 3309 & 41 \\
\hline $\mathrm{H}(39 \mathrm{~A})$ & 3800 & 3234 & 4461 & 37 \\
\hline $\mathrm{H}(39 \mathrm{~B})$ & 5076 & 3370 & 4771 & 37 \\
\hline $\mathrm{H}(40 \mathrm{~A})$ & 4107 & 2436 & 3077 & 29 \\
\hline $\mathrm{H}(40 \mathrm{~B})$ & 4669 & 1824 & 3900 & 29 \\
\hline $\mathrm{H}(41)$ & 5545 & 984 & 1018 & 25 \\
\hline $\mathrm{H}(42 \mathrm{~A})$ & 5415 & 3161 & 870 & 27 \\
\hline $\mathrm{H}(42 \mathrm{~B})$ & 6449 & 2609 & 617 & 27 \\
\hline $\mathrm{H}(43 \mathrm{~A})$ & 5385 & 1603 & -532 & 35 \\
\hline $\mathrm{H}(43 \mathrm{~B})$ & 5274 & 2812 & -708 & 35 \\
\hline $\mathrm{H}(44 \mathrm{~A})$ & 3559 & 2667 & -112 & 36 \\
\hline $\mathrm{H}(44 \mathrm{~B})$ & 3529 & 1779 & -885 & 36 \\
\hline $\mathrm{H}(45 \mathrm{~A})$ & 3811 & 516 & 220 & 37 \\
\hline $\mathrm{H}(45 \mathrm{~B})$ & 2785 & 1088 & 457 & 37 \\
\hline $\mathrm{H}(46 \mathrm{~A})$ & 3946 & 907 & 1804 & 33 \\
\hline $\mathrm{H}(46 \mathrm{~B})$ & 3879 & 2119 & 1601 & 33 \\
\hline $\mathrm{H}(47)$ & 4934 & 438 & 2975 & 23 \\
\hline $\mathrm{H}(48 \mathrm{~A})$ & 7055 & 395 & 3918 & 26 \\
\hline $\mathrm{H}(48 \mathrm{~B})$ & 6080 & 942 & 4302 & 26 \\
\hline $\mathrm{H}(49 \mathrm{~A})$ & 5027 & -692 & 4358 & 30 \\
\hline $\mathrm{H}(49 \mathrm{~B})$ & 6164 & -727 & 4952 & 30 \\
\hline
\end{tabular}




\begin{tabular}{lllll}
$\mathrm{H}(50 \mathrm{~A})$ & 5769 & -2258 & 4031 & 38 \\
$\mathrm{H}(50 \mathrm{~B})$ & 6852 & -1602 & 3735 & 38 \\
$\mathrm{H}(51 \mathrm{~A})$ & 4724 & -1602 & 2797 & 33 \\
$\mathrm{H}(51 \mathrm{~B})$ & 5712 & -2119 & 2406 & 33 \\
$\mathrm{H}(52 \mathrm{~A})$ & 5494 & -454 & 1741 & 26 \\
$\mathrm{H}(52 \mathrm{~B})$ & 6684 & -481 & 2246 & 26 \\
\hline
\end{tabular}

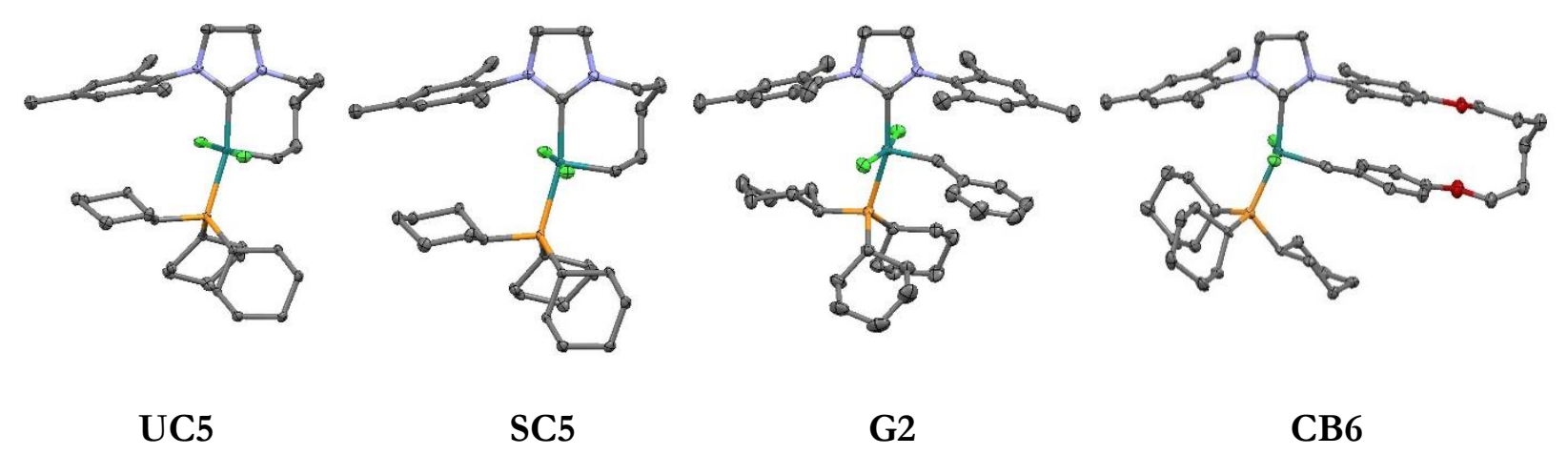

Figure S2. Solid-state structures of UC5, ${ }^{4} \mathbf{S C 5},{ }^{4} \mathbf{G} 2,{ }^{5}$ and $\mathbf{C B} 6$.

Table S6. Comparison of dihedral angles, bond angles, and bond length between $\mathbf{U C 5},{ }^{4} \mathbf{S C 5},{ }^{4} \mathbf{G} 2,{ }^{5}$ and CB6.

\begin{tabular}{ccccc}
\hline Dihedral Angles (deg) & UC5 & SC5 & G2 & CB6 \\
C12-Ru1-C2-C3 & 26.60 & 18.30 & 11.58 & 0.080 \\
N1-C1-Ru1-C2 & 162.2 & 160.0 & 167.5 & 179.9 \\
\hline & & & & \\
\hline Bond Angles (deg) & UC5 & SC5 & G2 & CB6 \\
C1-Ru1-P1 & 166.0 & 165.3 & 164.3 & 159.9 \\
N1-C1-Ru1 & 127.0 & 124.3 & 124.2 & 121.1 \\
N2-C1-Ru1 & 128.9 & 128.1 & 129.1 & 130.6 \\
N1-C1-N2 & 104.1 & 107.5 & 106.7 & 107.2 \\
\hline & & & & \\
\hline Bond Lengths (文) & UC5 & SC5 & G2 & CB6 \\
Ru1-C1 & 2.091 & 2.072 & 2.088 & 2.066 \\
Ru1-C2 & 1.806 & 1.821 & 1.836 & 1.845 \\
Ru1-P1 & 2.421 & 2.417 & 2.427 & 2.458 \\
\hline
\end{tabular}




\section{Solution-State Stability Study}

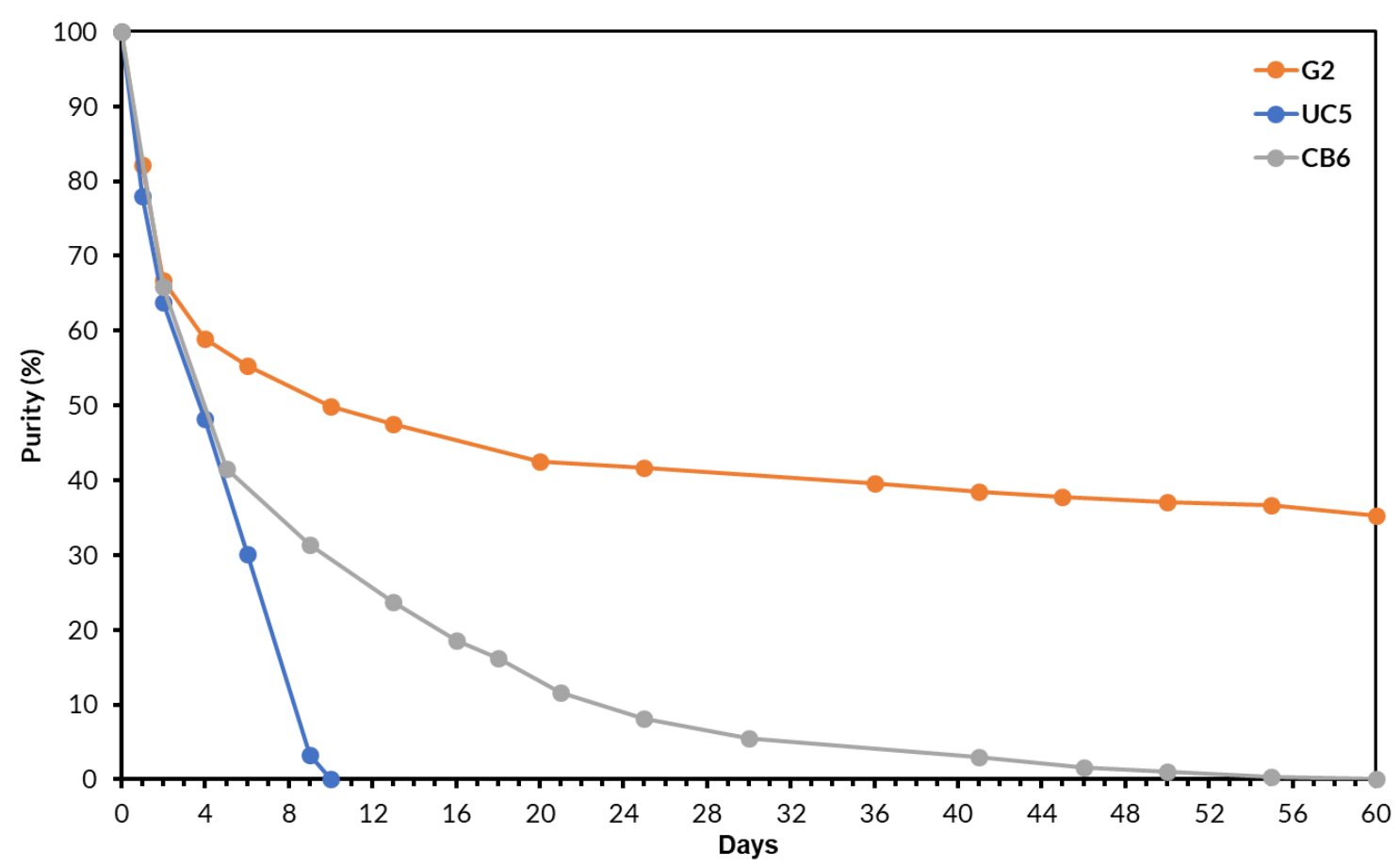

Figure S3. Stability of initiators $\mathbf{G} 2$, UC5, and $\mathbf{C B 6}$ in deuterated benzene $\left(\mathrm{C}_{6} \mathrm{D}_{6}\right)$ recorded over time using ${ }^{1} \mathrm{H}$ NMR spectroscopy (internal standard: naphthalene). Conditions:

[initiator $]_{0}=0.018 \mathrm{M}$ in $500 \mu \mathrm{L}$ of $\mathrm{C}_{6} \mathrm{D}_{6}$, sample prepared and sealed in a nitrogen-filled glovebox.

\section{Computational Strain Energy Analysis}

All calculations were performed using Gaussian16. All structures were optimized through DFT parameters at the B3LYP/6-31G(d)//LANL2DZ level of theory. See pages S62 - S72 for coordinates of optimized structures.

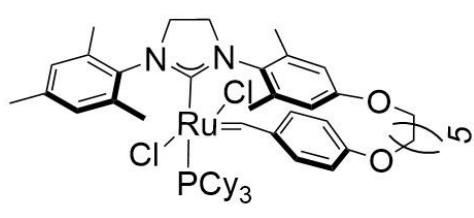

CB6

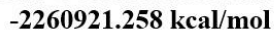
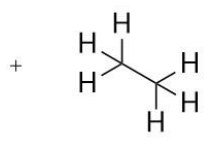

Ethane

SCF: -79.8127393795 A.U.

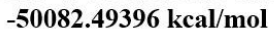

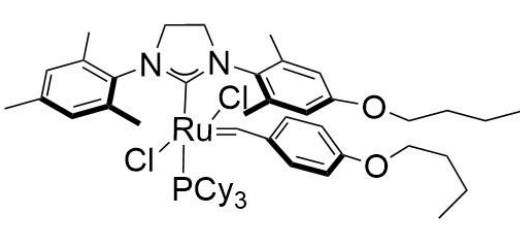

CB6-Open Adduct

SCF:-3682.89846429 A.U.

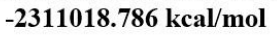

$E_{\text {strain }}=15.034 \mathrm{kcal} / \mathrm{mol}$

Figure S4. Homodesmotic reaction of $\mathbf{C B} 6$ to obtain strain energy. 


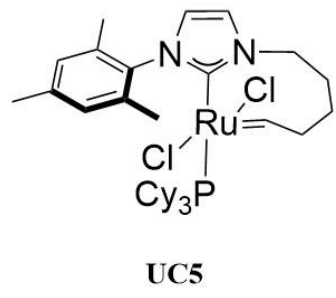

SCF: -2832.084986 A.U. -1777133.329 kcal/mol

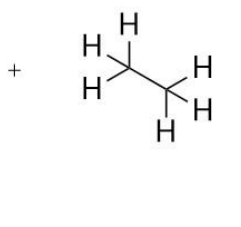

Ethane

SCF: -79.8127393795 A.U. $\mathbf{- 5 0 0 8 2 . 4 9 3 9 6} \mathrm{kcal} / \mathrm{mol}$

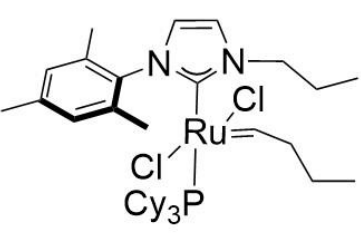

UC5-Open Adduct

SCF: -2911.908403 A.U. $-1827222.523 \mathrm{kcal} / \mathrm{mol}$

$E_{\text {strain }}=6.70004 \mathrm{kcal} / \mathrm{mol}$

Figure S5. Homodesmotic reaction of UC5 to obtain strain energy. 


\section{Long Term Storage Stability}

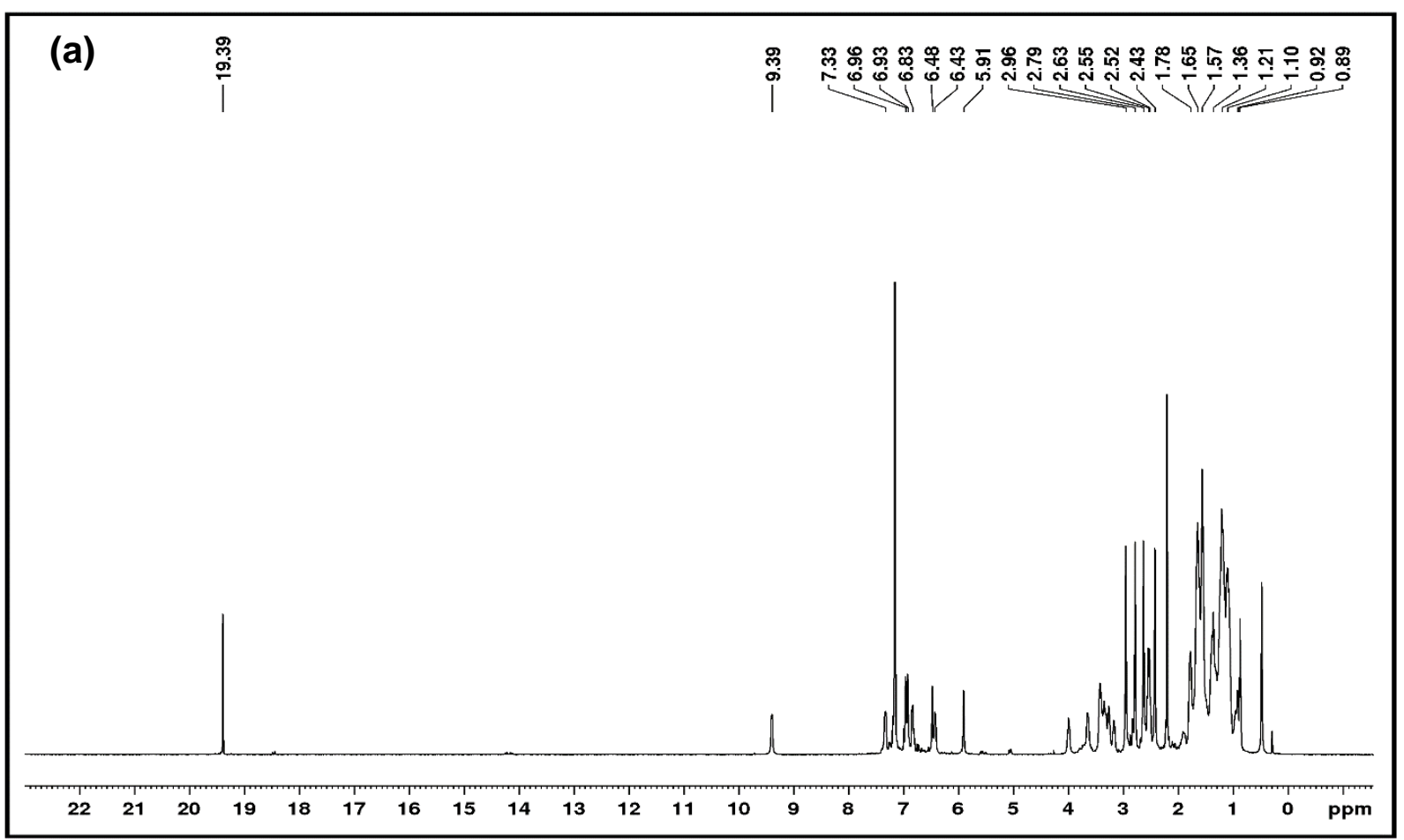

(b)

$\stackrel{\stackrel{8}{\circ}}{i}$

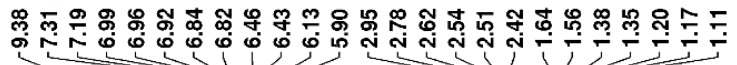
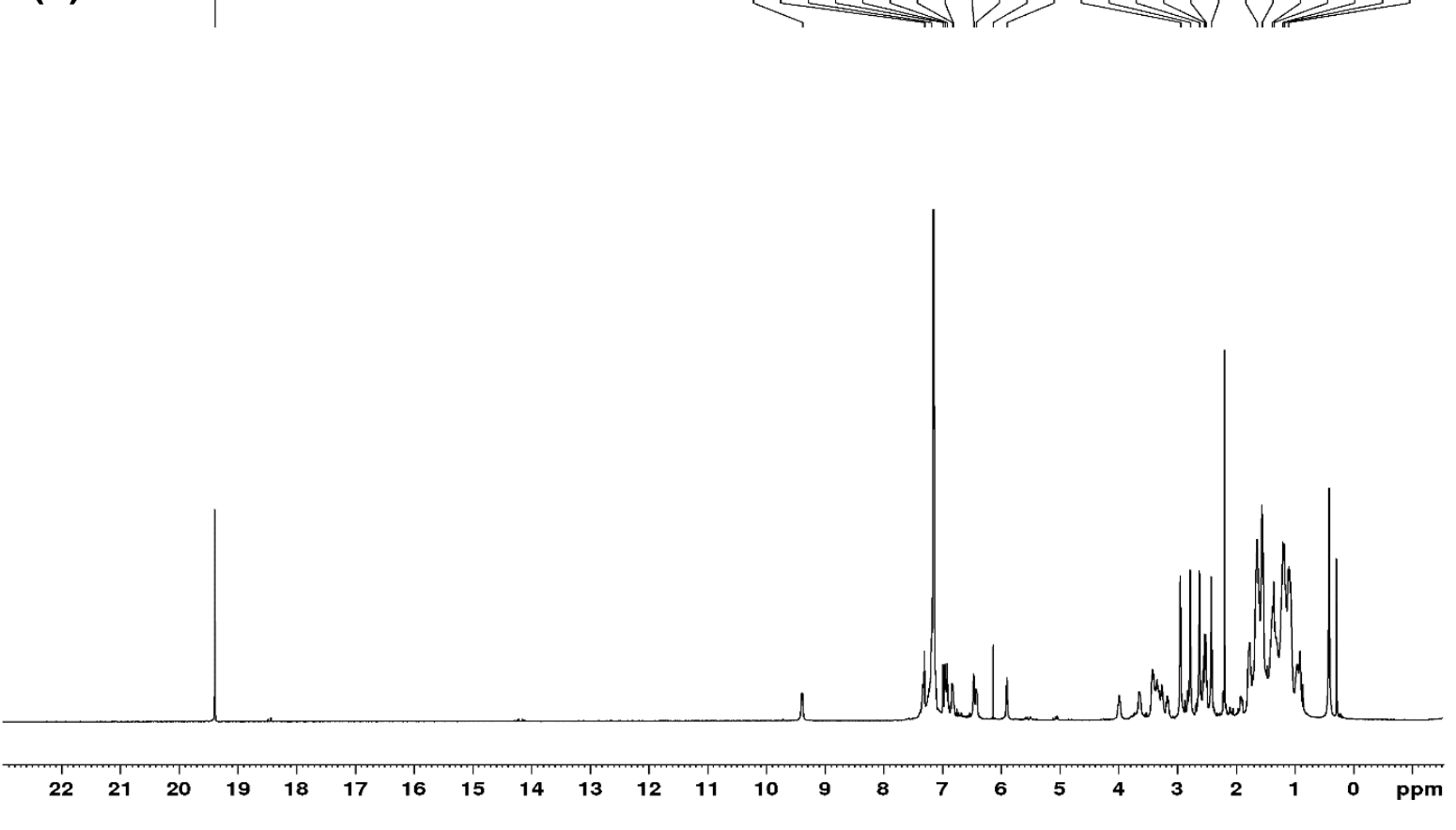

Figure S6. ${ }^{1} \mathrm{H}$ NMR (500 MHz, $\mathrm{C}_{6} \mathrm{D}_{6}$ ) stacked spectra of CB6: A) freshly prepared and $\mathbf{B}$ ) stored under nitrogen at $-20{ }^{\circ} \mathrm{C}$ for 5 months. 


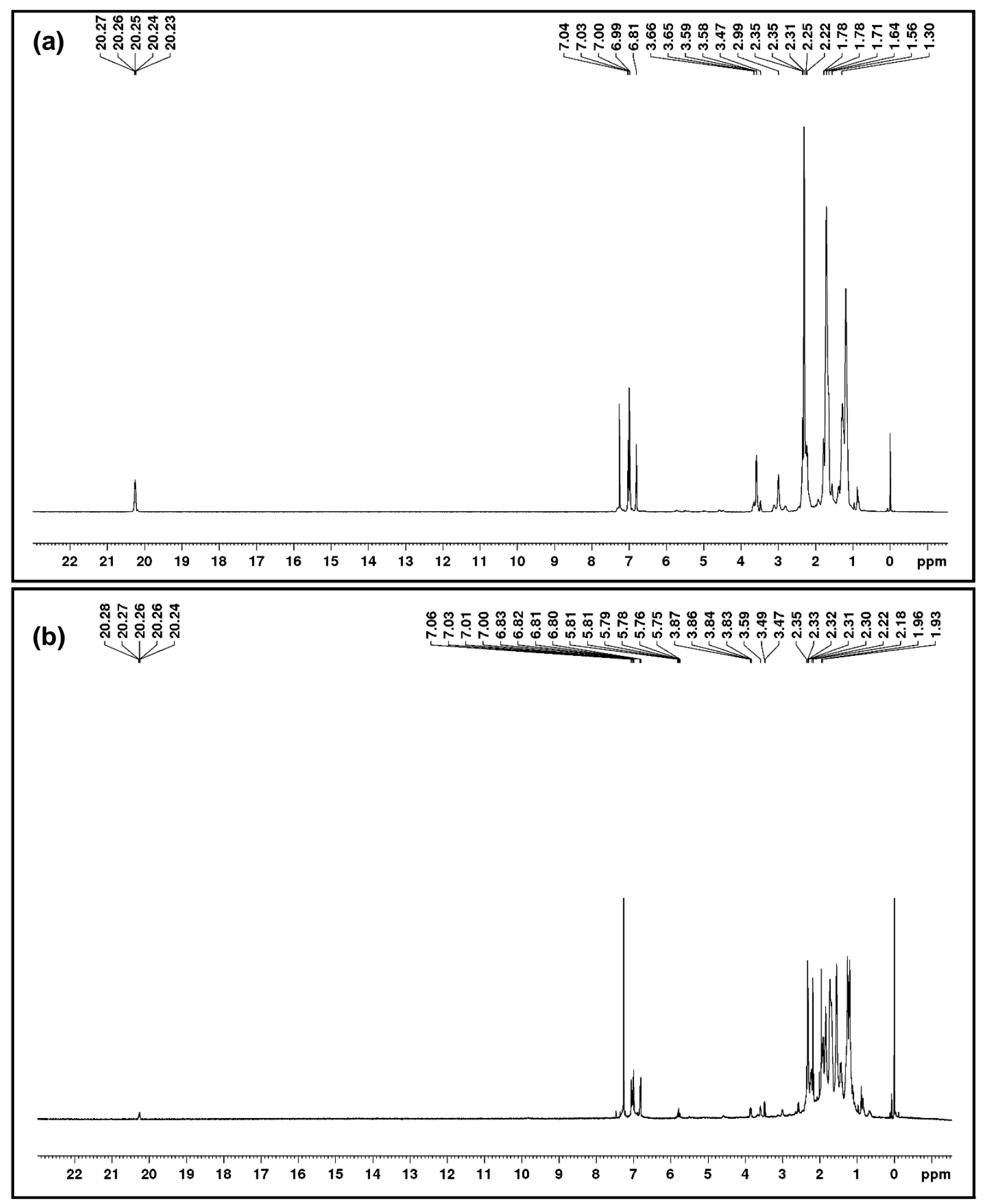

Figure S7. ${ }^{1} \mathrm{H}$ NMR $\left(500 \mathrm{MHz}, \mathrm{CDCl}_{3}\right)$ stacked spectra of UC5: A) freshly prepared and B) stored under nitrogen at $-20{ }^{\circ} \mathrm{C}$ for 3 months. 


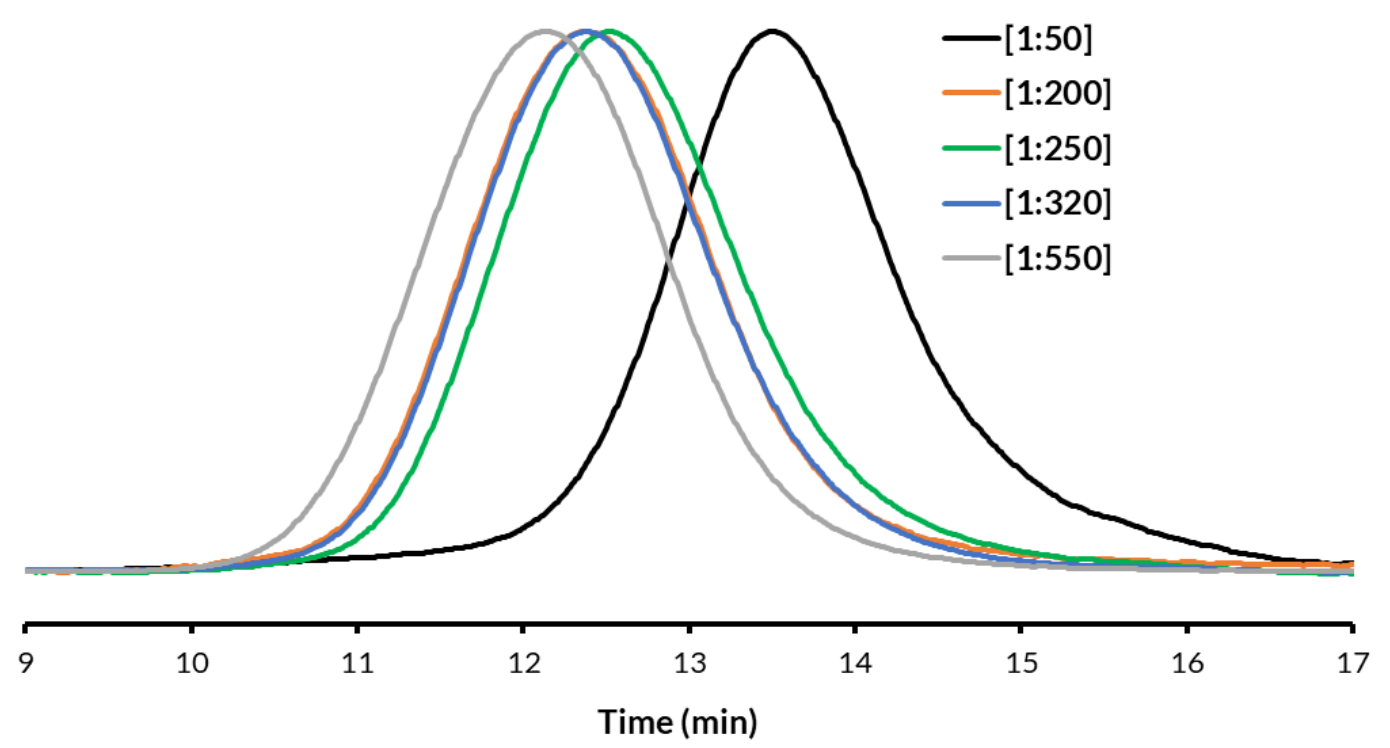

Figure S8. Evolution of GPC traces during REMP of $\mathbf{A c N b}$ with $\mathbf{C B 6}$ at various [CB6:AcNb] ratio. REMP condition: [monomer $]_{0}=0.2 \mathrm{M}, 55^{\circ} \mathrm{C}$.

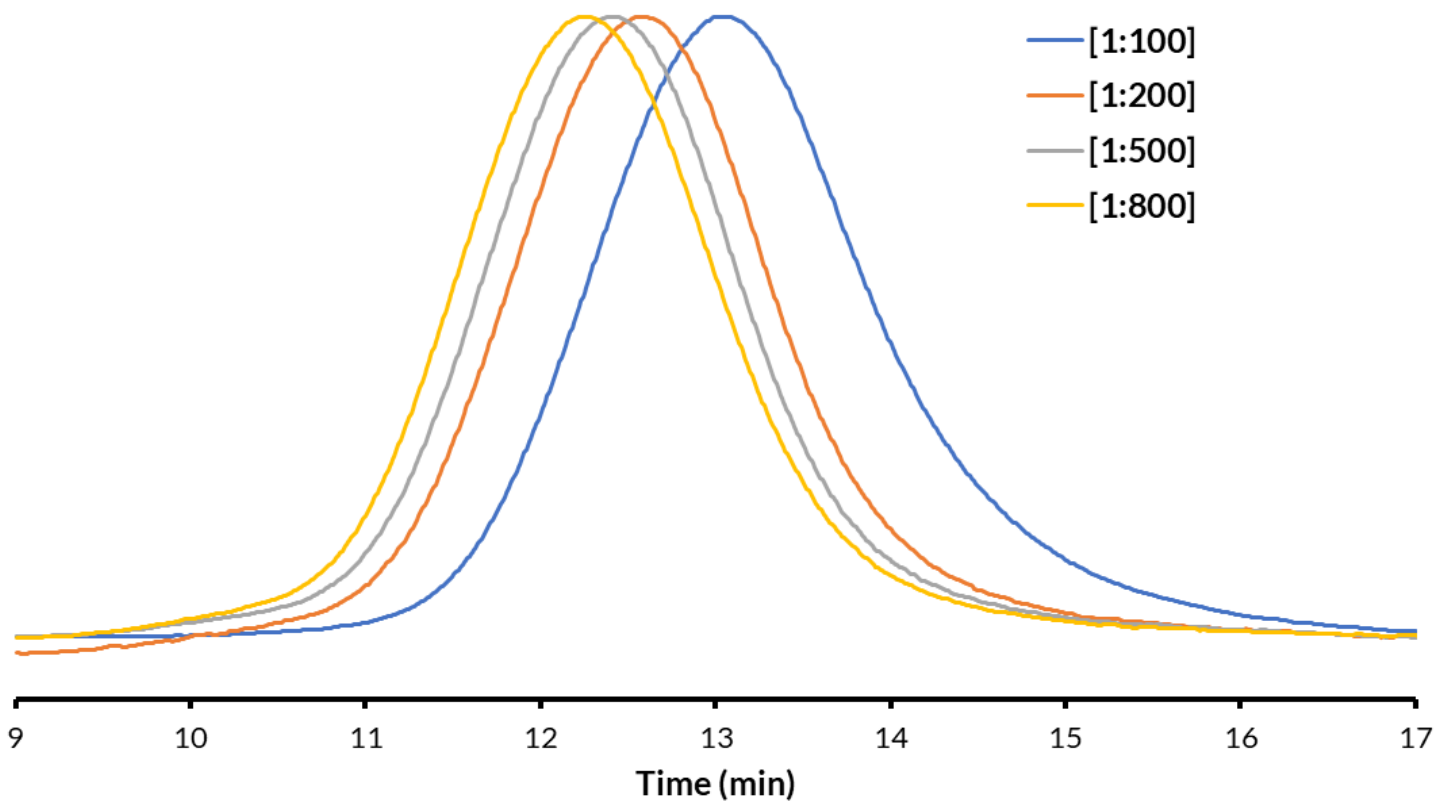

Figure S9. Evolution of GPC traces during REMP of $\mathbf{B n N b}$ with $\mathbf{C B 6}$ at various [CB6:BnNb] ratio. REMP condition: $[\text { monomer }]_{0}=0.2 \mathrm{M}, 55^{\circ} \mathrm{C}$. 


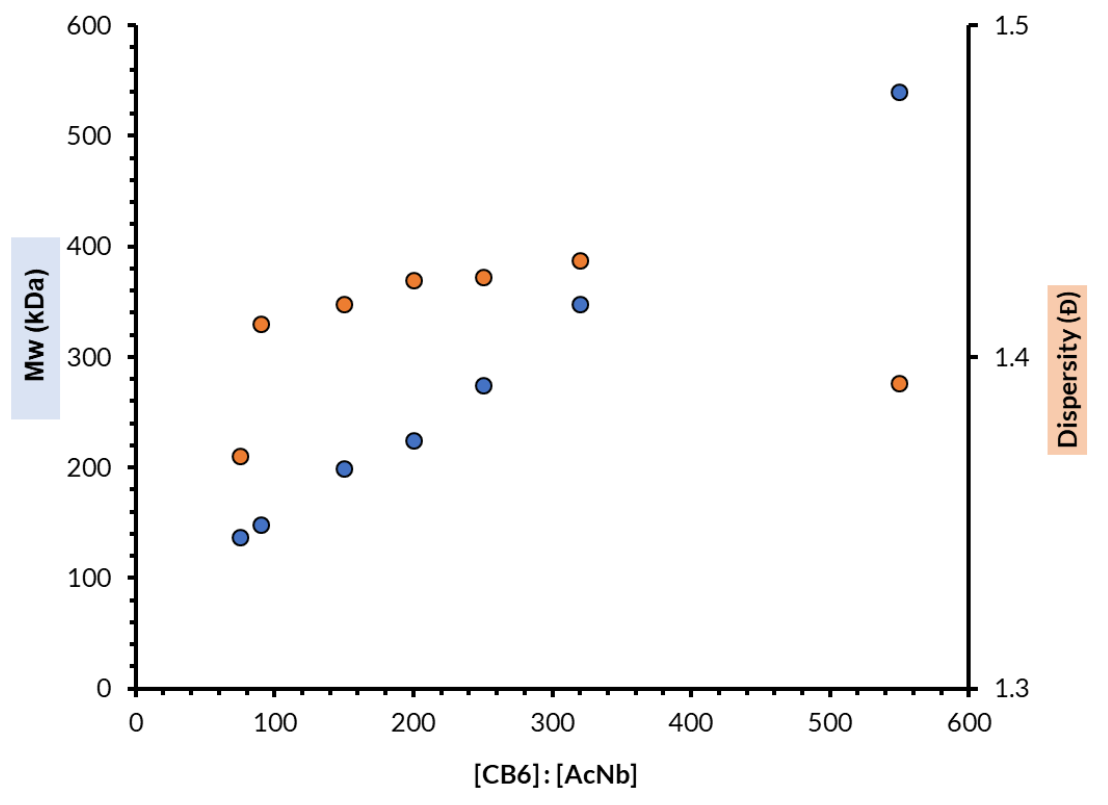

Figure S10. $\mathrm{M}_{\mathrm{w}}$ and $\left(\bigoplus=\mathrm{M}_{\mathrm{w}} / \mathrm{M}_{\mathrm{n}}\right)$ profiles versus $[\mathbf{C B 6} \mathbf{B n N b}$ of the monomer in living $\mathbf{A c N b}$ REMP

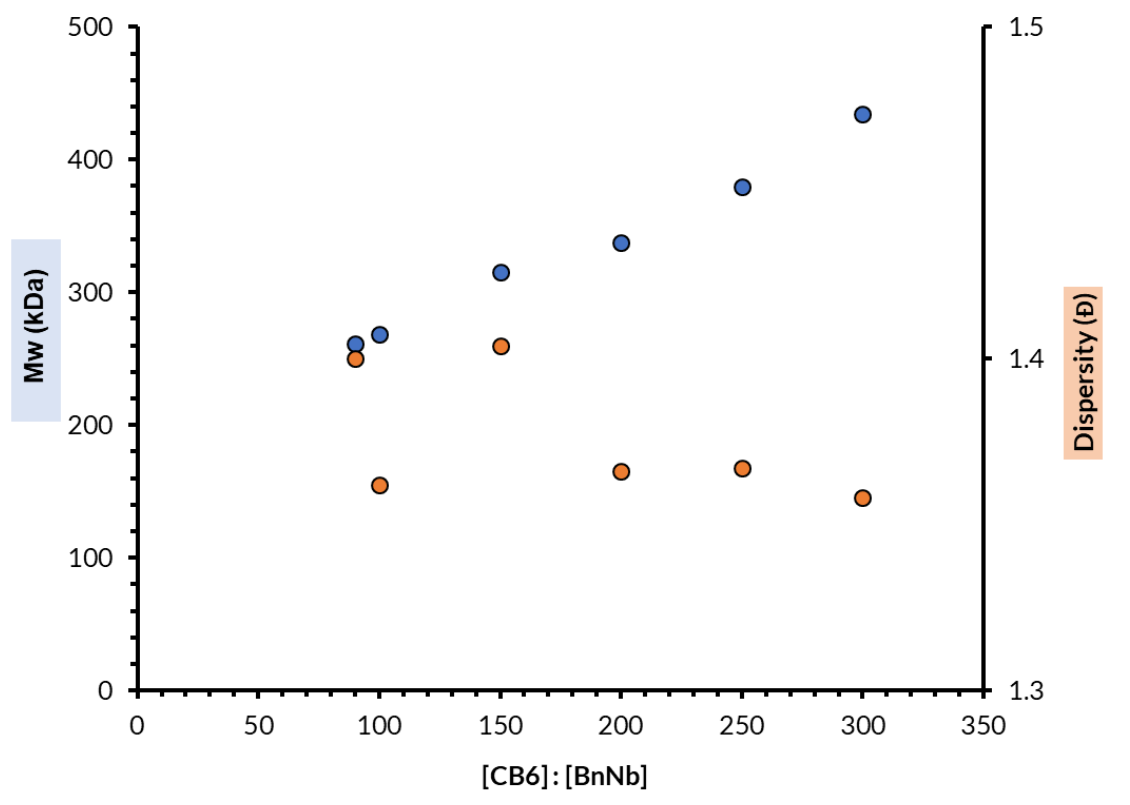

Figure S11. $\mathrm{M}_{\mathrm{w}}$ and $\left(\bigoplus=\mathrm{M}_{\mathrm{w}} / \mathrm{M}_{\mathrm{n}}\right)$ profiles versus $[\mathbf{C B 6}$ :BnNb] of the monomer in living $\mathbf{B n N b}$ REMP 


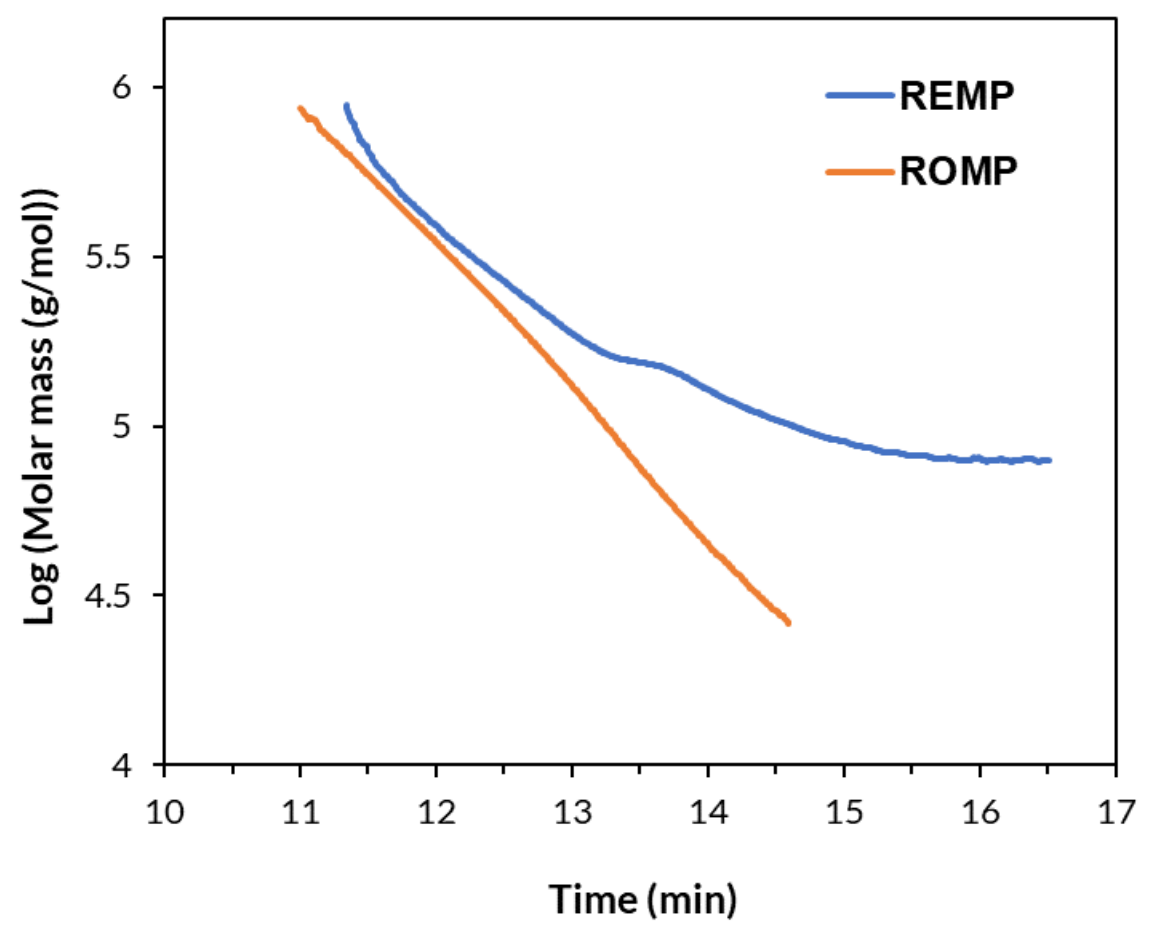

Figure S12. Molar mass versus time plot of REMP and ROMP for $\mathbf{B n N b}$.

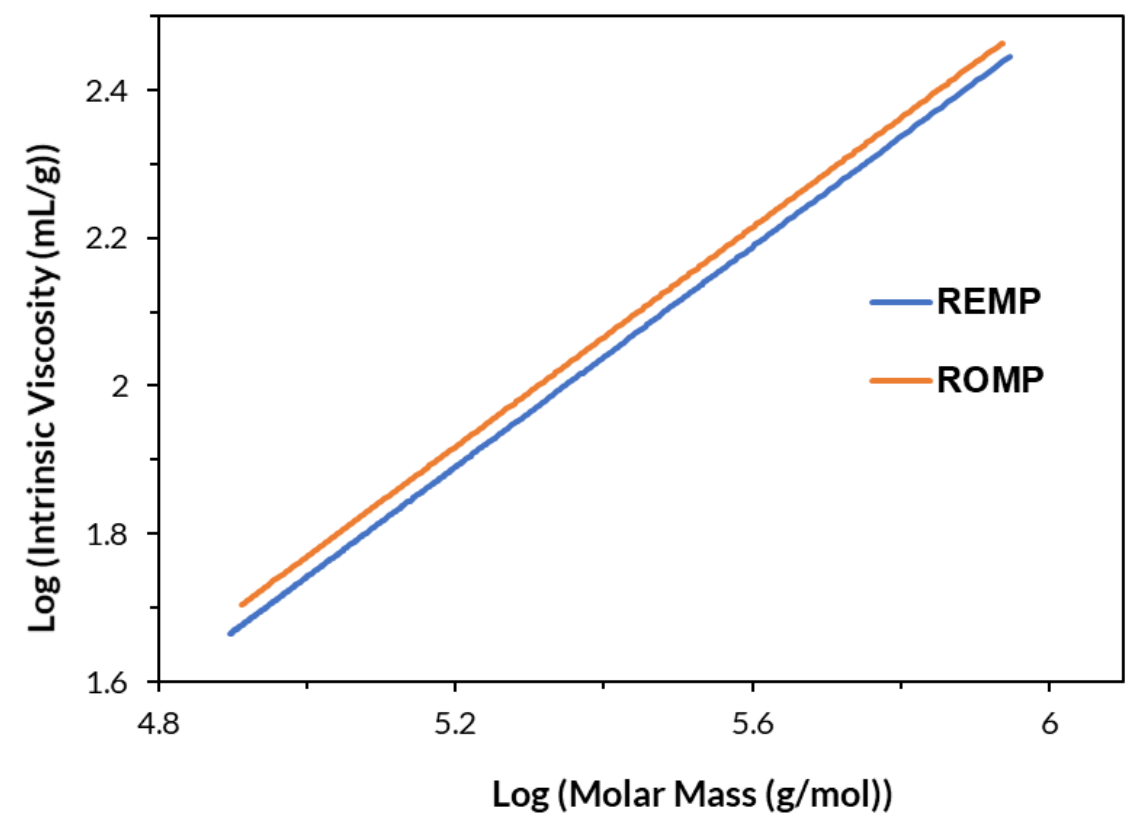

Figure S13. Intrinsic viscosity versus time plot of REMP and ROMP for $\mathbf{B n N b}$. 


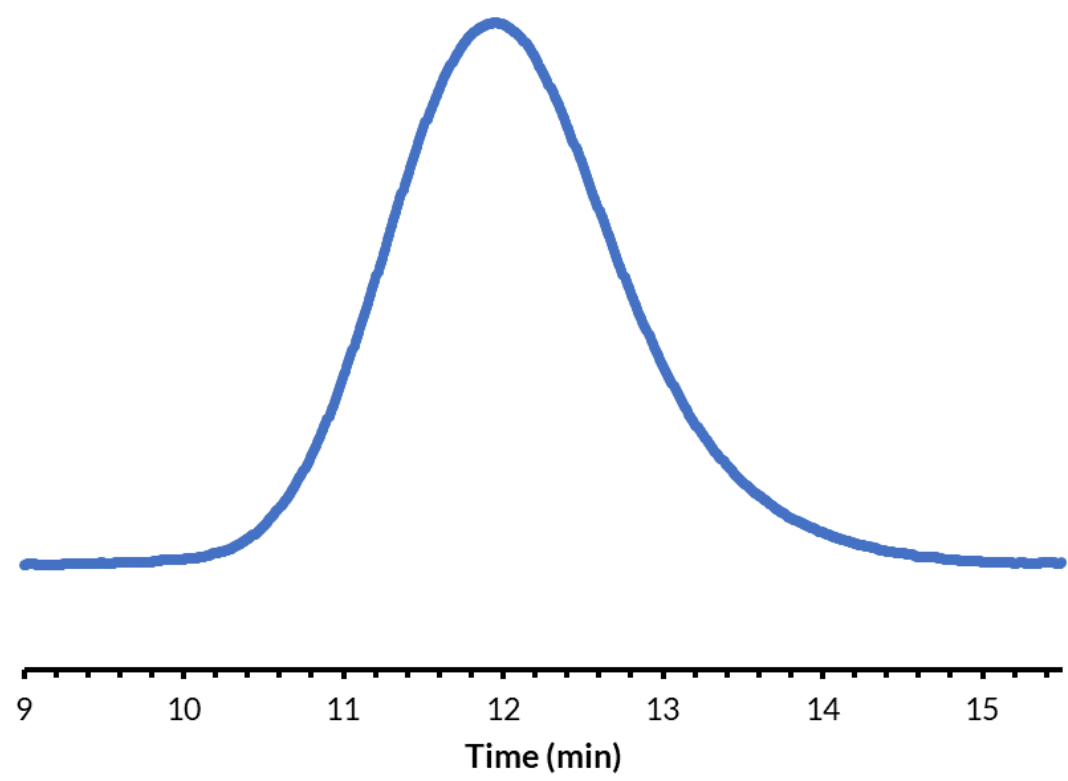

Figure S14. GPC trace (RI) of benchtop REMP of AcNb with $\mathbf{C B} 6$ in air. $\mathbf{M}_{\mathrm{w}}: 583 \mathrm{kDa}, \mathbf{M}_{\mathrm{n}}: 388$ $\mathrm{kDa}, \mathrm{M}_{\mathrm{w}} / \mathrm{M}_{\mathrm{n}}$ : 1.50. REMP condition: [CB6:AcNb] $=1: 200$, [monomer] $]_{0}=0.2 \mathrm{M}, 55^{\circ} \mathrm{C}$ for $12 \mathrm{hr}$.

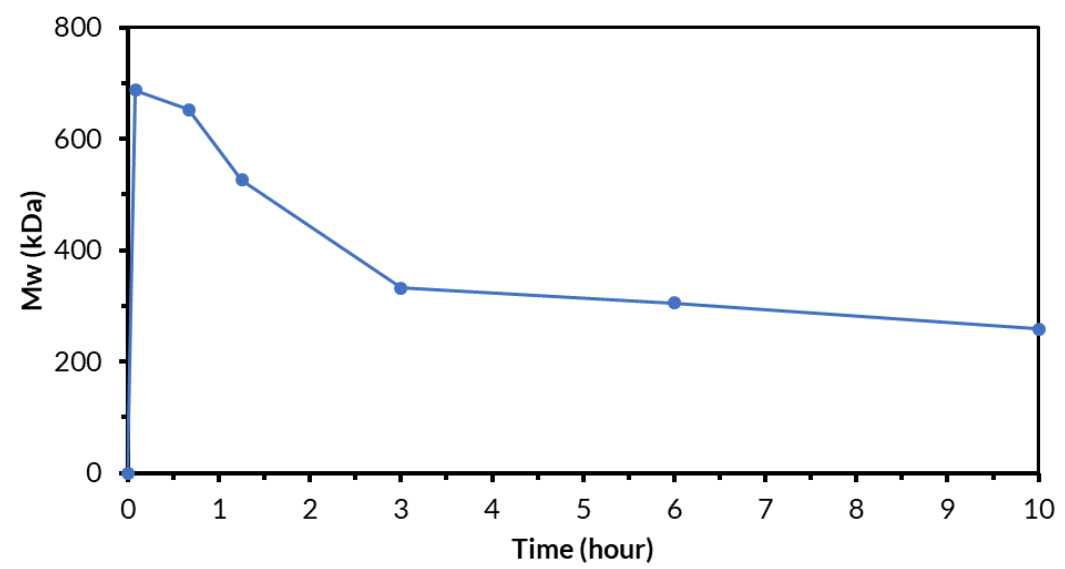

Figure S15. Evolution of $\mathrm{M}_{\mathrm{w}}$ with time as assessed by GPC-MALS-IV. REMP conditions: $[\mathbf{C B 6}: \mathbf{A c N b}]=1: 200,[\text { monomer }]_{0}=0.2 \mathrm{M}, 55^{\circ} \mathrm{C}$. 


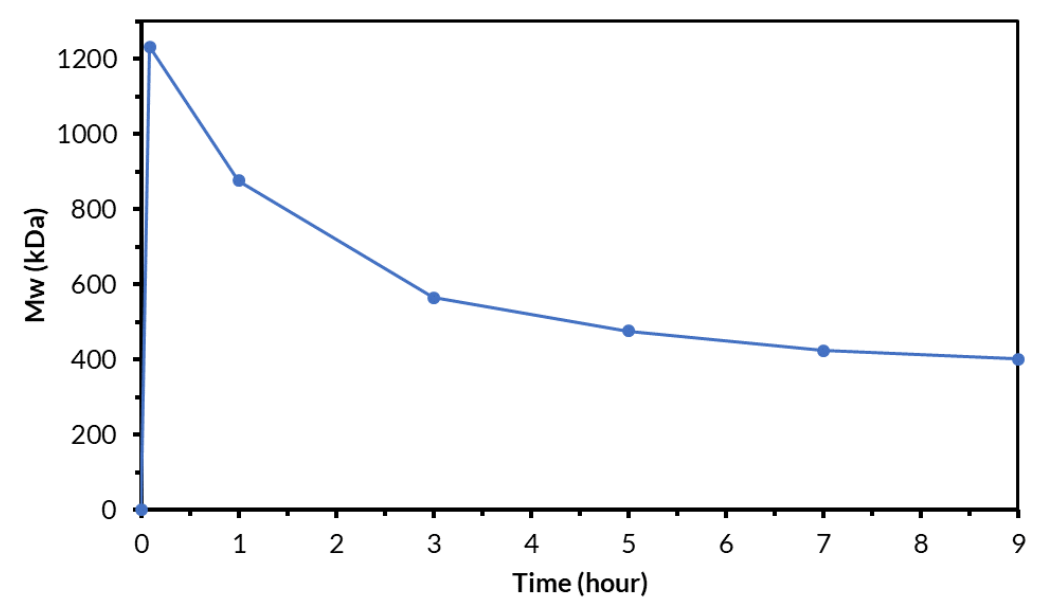

Figure S16. Evolution of $\mathrm{M}_{\mathrm{w}}$ with time as assessed by GPC-MALS-IV. REMP conditions: $\left[\right.$ CB6:BnNb] $=1: 200,[\text { monomer }]_{0}=0.2 \mathrm{M}, 55^{\circ} \mathrm{C}$.

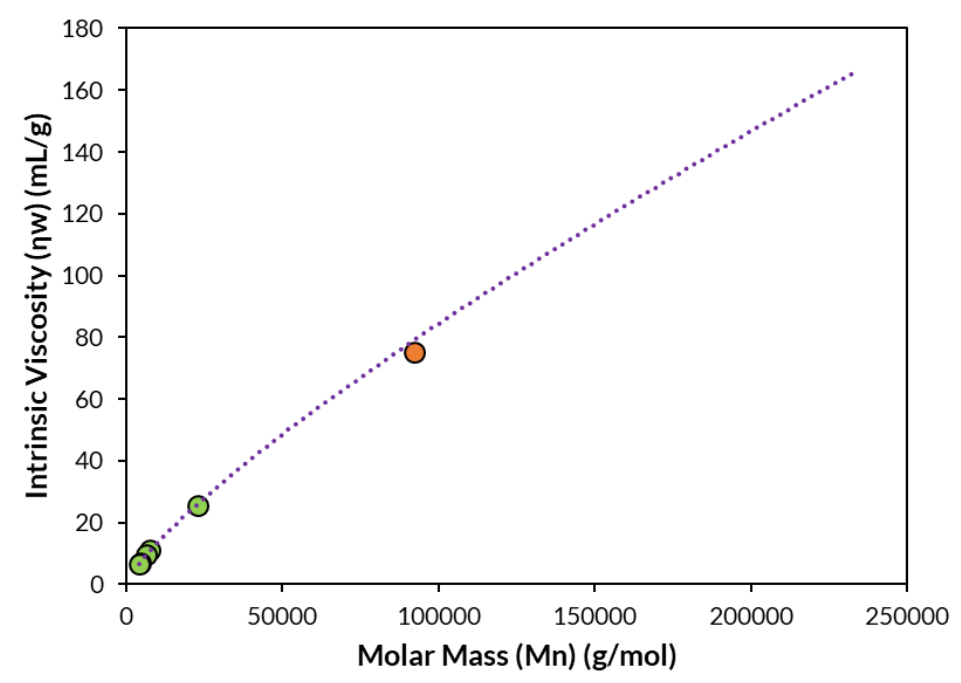

Figure S17. Mark-Houwink-Sakurada plot obtained during ROMP of $\mathbf{A c N b}$ with $\mathbf{G} 2$ in the presence of varying concentrations of 3-hexene CTA. [CTA]: $[\mathrm{Ru}]=0: 1 \rightarrow$ 10:1. Orange point indicates data when no CTA was added (i.e., [CTA]:[Ru] = 0:1). 


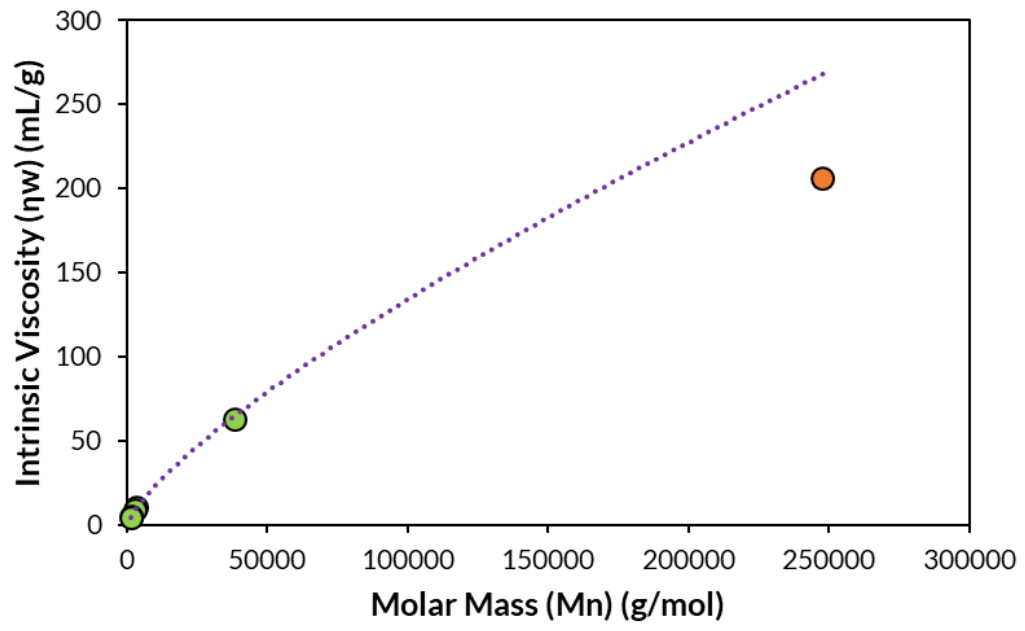

Figure S18. Mark-Houwink-Sakurada plot obtained during REMP of AcNb with UC5 in the presence of varying concentrations of 3-hexene CTA. [CTA]:[Ru] $=0: 1 \rightarrow 10: 1$. Orange point indicates data when no CTA was added (i.e., [CTA]:[Ru] = 0:1).

\section{$\underline{\text { References }}$}

(1) Roche, S. P.; Teyssot, M-L.; Gautier, A. Synthesis of 1,2-Diamines Under Environmentally Benign Conditions: Applications for the Preparation of Imidazolinium Salts. Tet. Lett. 2010, 51, 1265-1268. https://doi.org/10.1016/j.tetlet.2009.12.072.

(2) Occhipinti, G.; Bjørsvik, H. R.; Törnroos, K. W.; Fürstner, A.; Jensen, V. R. The First Imidazolium-Substituted Metal Alkylidene. Organometallics 2007, 26, 4383-4385. https://doi.org/10.1021/om700590v.

(3) Lin, Y.; Zhang, Y.; Wang, Z.; Craig, S. L. Dynamic Memory Effects in the Mechanochemistry of Cyclic Polymers. J. Am. Chem. Soc. 2019, 141, 10943-10947. https://doi.org/10.1021/jacs.9b03564.

(4) Boydston, A. J.; Xia, Y.; Kornfield, J. A.; Gorodetskaya, I. A.; Grubbs, R. H. Cyclic Ruthenium-Alkylidene Catalysts for Ring-Expansion Metathesis Polymerization. J. Am. Chem. Soc. 2008, 130, 12775-12782. https://doi.org/10.1021/ja8037849

(5) Lehman, S. E.; Wagener, K. B. Synthesis of Ruthenium Olefin Metathesis Catalysts with Linear Alkyl Carbene Complexes. Organometallics 2005, 24, 1477-1482. https://doi.org/10.1021/om049176q. 


\section{$\underline{\text { Raw NMR Kinetics Data }}$}

Table S7. ${ }^{1} \mathrm{H}$ NMR kinetics data for polymerization of $\mathbf{A c N b}$ initiated by $\mathbf{G} 2$ (DCE- $d 4$ at $55{ }^{\circ} \mathrm{C}$ )

\begin{tabular}{|c|c|c|c|c|}
\hline time (sec) & integral & normalized integral & conversion & conversion $(\%)$ \\
\hline 0 & 1.5917 & 1 & 0 & 0 \\
\hline 6 & 0.3531 & 0.221838286 & 0.778161714 & 77.81617139 \\
\hline 7 & 0.179 & 0.112458378 & 0.887541622 & 88.75416222 \\
\hline 8 & 0.1106 & 0.069485456 & 0.930514544 & 93.05145442 \\
\hline 9 & 0.0684 & 0.042972922 & 0.957027078 & 95.7027078 \\
\hline 10 & 0.0453 & 0.028460137 & 0.971539863 & 97.1539863 \\
\hline 11 & 0.0259 & 0.016271911 & 0.983728089 & 98.37280895 \\
\hline 12 & 0.0118 & 0.007413457 & 0.992586543 & 99.25865427 \\
\hline 13 & 0 & 0 & 1 & 100 \\
\hline 14 & 0 & 0 & 1 & 100 \\
\hline 15 & 0 & 0 & 1 & 100 \\
\hline 16 & 0 & 0 & 1 & 100 \\
\hline 17 & 0 & 0 & 1 & 100 \\
\hline 18 & 0 & 0 & 1 & 100 \\
\hline 19 & 0 & 0 & 1 & 100 \\
\hline 20 & 0 & 0 & 1 & 100 \\
\hline 21 & 0 & 0 & 1 & 100 \\
\hline 22 & 0 & 0 & 1 & 100 \\
\hline 23 & 0 & 0 & 1 & 100 \\
\hline 24 & 0 & 0 & 1 & 100 \\
\hline 25 & 0 & 0 & 1 & 100 \\
\hline 26 & 0 & 0 & 1 & 100 \\
\hline 27 & 0 & 0 & 1 & 100 \\
\hline 28 & 0 & 0 & 1 & 100 \\
\hline 29 & 0 & 0 & 1 & 100 \\
\hline 30 & 0 & 0 & 1 & 100 \\
\hline 31 & 0 & 0 & 1 & 100 \\
\hline 32 & 0 & 0 & 1 & 100 \\
\hline 33 & 0 & 0 & 1 & 100 \\
\hline 34 & 0 & 0 & 1 & 100 \\
\hline 35 & 0 & 0 & 1 & 100 \\
\hline 36 & 0 & 0 & 1 & 100 \\
\hline 37 & 0 & 0 & 1 & 100 \\
\hline 38 & 0 & 0 & 1 & 100 \\
\hline 39 & 0 & 0 & 1 & 100 \\
\hline 40 & 0 & 0 & 1 & 100 \\
\hline
\end{tabular}


Table S8. ${ }^{1} \mathrm{H}$ NMR kinetics data for polymerization of AcNb initiated by UC5 (DCE- $d 4$ at $55{ }^{\circ} \mathrm{C}$ )

\begin{tabular}{|c|c|c|c|c|}
\hline time (sec) & integral & normalize integral & conversion & conversion $(\%)$ \\
\hline 0 & 1.7853 & 1 & 0 & 0 \\
\hline 30 & 1.7661 & 0.989245505 & 0.010754495 & 1.075449504 \\
\hline 55 & 1.7648 & 0.988517336 & 0.011482664 & 1.148266398 \\
\hline 80 & 1.7603 & 0.985996751 & 0.014003249 & 1.400324875 \\
\hline 105 & 1.7552 & 0.983140089 & 0.016859911 & 1.68599115 \\
\hline 130 & 1.7487 & 0.979499244 & 0.020500756 & 2.050075618 \\
\hline 155 & 1.7357 & 0.972217554 & 0.027782446 & 2.778244553 \\
\hline 180 & 1.7256 & 0.966560242 & 0.033439758 & 3.343975802 \\
\hline 205 & 1.7325 & 0.970425139 & 0.029574861 & 2.957486137 \\
\hline 230 & 1.7102 & 0.957934241 & 0.042065759 & 4.206575926 \\
\hline 255 & 1.6982 & 0.951212681 & 0.048787319 & 4.878731866 \\
\hline 280 & 1.6841 & 0.943314849 & 0.056685151 & 5.668515096 \\
\hline 305 & 1.6859 & 0.944323083 & 0.055676917 & 5.567691704 \\
\hline 330 & 1.6841 & 0.943314849 & 0.056685151 & 5.668515096 \\
\hline 355 & 1.6761 & 0.938833809 & 0.061166191 & 6.116619056 \\
\hline 387 & 1.6531 & 0.925950821 & 0.074049179 & 7.404917941 \\
\hline 425 & 1.6489 & 0.923598275 & 0.076401725 & 7.64017252 \\
\hline 470 & 1.6381 & 0.917548871 & 0.082451129 & 8.245112866 \\
\hline 527 & 1.6235 & 0.909370974 & 0.090629026 & 9.062902593 \\
\hline 721 & 1.6009 & 0.896712037 & 0.103287963 & 10.32879628 \\
\hline 915 & 1.5757 & 0.882596762 & 0.117403238 & 11.74032376 \\
\hline 1303 & 1.5296 & 0.856774772 & 0.143225228 & 14.32252283 \\
\hline 1497 & 1.5035 & 0.84215538 & 0.15784462 & 15.784462 \\
\hline 1691 & 1.4781 & 0.827928079 & 0.172071921 & 17.20719207 \\
\hline 1885 & 1.4683 & 0.822438806 & 0.177561194 & 17.75611942 \\
\hline 2079 & 1.4561 & 0.81560522 & 0.18439478 & 18.43947796 \\
\hline 2273 & 1.4391 & 0.806083011 & 0.193916989 & 19.39169887 \\
\hline 2661 & 1.4095 & 0.789503165 & 0.210496835 & 21.04968353 \\
\hline 2855 & 1.4093 & 0.789391139 & 0.210608861 & 21.06088613 \\
\hline 3049 & 1.3956 & 0.781717358 & 0.218282642 & 21.82826416 \\
\hline 3437 & 1.3845 & 0.775499916 & 0.224500084 & 22.4500084 \\
\hline 3631 & 1.37 & 0.767378032 & 0.232621968 & 23.26219683 \\
\hline 3825 & 1.3612 & 0.762448888 & 0.237551112 & 23.75511119 \\
\hline 4019 & 1.3477 & 0.754887134 & 0.245112866 & 24.51128662 \\
\hline 4407 & 1.3499 & 0.75611942 & 0.24388058 & 24.38805803 \\
\hline 4601 & 1.3292 & 0.74452473 & 0.25547527 & 25.54752703 \\
\hline 4989 & 1.3402 & 0.750686159 & 0.249313841 & 24.93138408 \\
\hline 5183 & 1.3296 & 0.744748782 & 0.255251218 & 25.52512183 \\
\hline 5377 & 1.315 & 0.736570884 & 0.263429116 & 26.34291156 \\
\hline 5571 & 1.3016 & 0.729065143 & 0.270934857 & 27.09348569 \\
\hline
\end{tabular}




\begin{tabular}{|l|l|l|l|l|}
\hline 5959 & 1.3071 & 0.732145858 & 0.267854142 & 26.78541422 \\
\hline 6153 & 1.2857 & 0.720159077 & 0.279840923 & 27.98409231 \\
\hline 6347 & 1.2951 & 0.725424298 & 0.274575702 & 27.45757016 \\
\hline 6541 & 1.2763 & 0.714893855 & 0.285106145 & 28.51061446 \\
\hline 6735 & 1.2637 & 0.707836218 & 0.292163782 & 29.2163782 \\
\hline 6929 & 1.2645 & 0.708284322 & 0.291715678 & 29.1715678 \\
\hline 7123 & 1.2543 & 0.702570996 & 0.297429004 & 29.74290035 \\
\hline 7317 & 1.2614 & 0.706547919 & 0.293452081 & 29.34520809 \\
\hline 7511 & 1.2562 & 0.703635243 & 0.296364757 & 29.63647566 \\
\hline 7705 & 1.2401 & 0.694617151 & 0.305382849 & 30.53828488 \\
\hline 7899 & 1.2334 & 0.690864281 & 0.309135719 & 30.91357195 \\
\hline 8213 & 1.2279 & 0.687783566 & 0.312216434 & 31.22164342 \\
\hline 8527 & 1.2097 & 0.677589201 & 0.322410799 & 32.24107993 \\
\hline 8841 & 1.2264 & 0.686943371 & 0.313056629 & 31.30566291 \\
\hline 9155 & 1.217 & 0.681678149 & 0.318321851 & 31.83218507 \\
\hline 9469 & 1.1981 & 0.671091693 & 0.328908307 & 32.89083067 \\
\hline 10097 & 1.1891 & 0.666050524 & 0.333949476 & 33.39494763 \\
\hline 10411 & 1.1817 & 0.661905562 & 0.338094438 & 33.80944379 \\
\hline
\end{tabular}

Table S9. ${ }^{1} \mathrm{H}$ NMR kinetics data for polymerization of $\mathbf{A c N b}$ initiated by $\mathbf{C B 6}\left(\mathrm{DCE}-d 4\right.$ at $\left.55{ }^{\circ} \mathrm{C}\right)$

\begin{tabular}{|l|l|l|l|l|}
\hline time (sec) & integral & normalize integral & conversion & conversion (\%) \\
\hline 0 & 3.1873 & 1 & 0 & 0 \\
\hline 12 & 2.4871 & 0.780315628 & 0.219684 & 21.96843724 \\
\hline 15 & 2.2839 & 0.716562608 & 0.283437 & 28.34373922 \\
\hline 21 & 1.8223 & 0.571737835 & 0.428262 & 42.82621655 \\
\hline 24 & 1.7171 & 0.538731842 & 0.461268 & 46.1268158 \\
\hline 27 & 1.4718 & 0.46177015 & 0.53823 & 53.82298497 \\
\hline 33 & 1.0921 & 0.342641107 & 0.657359 & 65.73588931 \\
\hline 36 & 0.9092 & 0.285257114 & 0.714743 & 71.47428858 \\
\hline 39 & 0.8081 & 0.253537477 & 0.746463 & 74.64625231 \\
\hline 42 & 0.6798 & 0.213283971 & 0.786716 & 78.67160292 \\
\hline 45 & 0.6239 & 0.195745615 & 0.804254 & 80.42543846 \\
\hline 48 & 0.561 & 0.176011044 & 0.823989 & 82.39889562 \\
\hline 51 & 0.4945 & 0.15514699 & 0.844853 & 84.48530104 \\
\hline 54 & 0.4493 & 0.140965708 & 0.859034 & 85.90342923 \\
\hline 57 & 0.4173 & 0.130925862 & 0.869074 & 86.9074138 \\
\hline 60 & 0.3917 & 0.122893986 & 0.877106 & 87.71060145 \\
\hline 63 & 0.3398 & 0.106610611 & 0.893389 & 89.33893891 \\
\hline 66 & 0.3054 & 0.095817777 & 0.904182 & 90.41822232 \\
\hline 69 & 0.2784 & 0.087346657 & 0.912653 & 91.2653343 \\
\hline 72 & 0.256 & 0.080318765 & 0.919681 & 91.96812349 \\
\hline 75 & 0.2365 & 0.074200734 & 0.925799 & 92.57992658 \\
\hline
\end{tabular}




\begin{tabular}{|c|c|c|c|c|}
\hline 78 & 0.2152 & 0.067517962 & 0.932482 & 93.24820381 \\
\hline 81 & 0.2059 & 0.064600132 & 0.9354 & 93.53998682 \\
\hline 84 & 0.1816 & 0.056976124 & 0.943024 & 94.3023876 \\
\hline 87 & 0.1525 & 0.047846139 & 0.952154 & 95.21538606 \\
\hline 90 & 0.1343 & 0.042135977 & 0.957864 & 95.78640228 \\
\hline 93 & 0.1221 & 0.038308286 & 0.961692 & 96.1691714 \\
\hline 96 & 0.1128 & 0.035390456 & 0.96461 & 96.46095441 \\
\hline 99 & 0.0961 & 0.030150911 & 0.969849 & 96.98490886 \\
\hline 102 & 0.0905 & 0.028393938 & 0.971606 & 97.16060616 \\
\hline 105 & 0.0803 & 0.025193738 & 0.974806 & 97.48062624 \\
\hline 108 & 0.0747 & 0.023436765 & 0.976563 & 97.65632353 \\
\hline 111 & 0.0655 & 0.020550309 & 0.97945 & 97.9449691 \\
\hline 114 & 0.0542 & 0.017004989 & 0.982995 & 98.29950115 \\
\hline 117 & 0.0482 & 0.015122517 & 0.984877 & 98.48774825 \\
\hline 120 & 0.0428 & 0.013428294 & 0.986572 & 98.65717065 \\
\hline 123 & 0.0354 & 0.011106579 & 0.988893 & 98.88934208 \\
\hline 126 & 0.0289 & 0.009067236 & 0.990933 & 99.09327644 \\
\hline 129 & 0.0263 & 0.008251498 & 0.991749 & 99.17485019 \\
\hline 132 & 0.0175 & 0.005490541 & 0.994509 & 99.45094594 \\
\hline 135 & 0.0196 & 0.006149405 & 0.993851 & 99.38505945 \\
\hline 138 & 0.0159 & 0.004988548 & 0.995011 & 99.50114517 \\
\hline 141 & 0 & 0 & 1 & 100 \\
\hline 144 & 0 & 0 & 1 & 100 \\
\hline 147 & 0 & 0 & 1 & 100 \\
\hline 150 & 0 & 0 & 1 & 100 \\
\hline 153 & 0 & 0 & 1 & 100 \\
\hline 156 & 0 & 0 & 1 & 100 \\
\hline 159 & 0 & 0 & 1 & 100 \\
\hline 162 & 0 & 0 & 1 & 100 \\
\hline 165 & 0 & 0 & 1 & 100 \\
\hline 168 & 0 & 0 & 1 & 100 \\
\hline 171 & 0 & 0 & 1 & 100 \\
\hline 174 & 0 & 0 & 1 & 100 \\
\hline 177 & 0 & 0 & 1 & 100 \\
\hline 180 & 0 & 0 & 1 & 100 \\
\hline 183 & 0 & 0 & 1 & 100 \\
\hline 186 & 0 & 0 & 1 & 100 \\
\hline 189 & 0 & 0 & 1 & 100 \\
\hline 192 & 0 & 0 & 1 & 100 \\
\hline 195 & 0 & 0 & 1 & 100 \\
\hline 198 & 0 & 0 & 1 & 100 \\
\hline 201 & 0 & 0 & 1 & 100 \\
\hline 204 & 0 & 0 & 1 & 100 \\
\hline
\end{tabular}




\section{$\underline{\text { DFT Coordinates }}$}

UC5, B3LYP/6-31G(d)//LANL2DZ (Energy = -2832.08498610 Hartrees)

$\begin{array}{llll}\mathrm{Ru} & -0.28911 & -0.96296 & -0.14285 \\ \mathrm{Cl} & -0.65343 & -0.58917 & 2.27549 \\ \mathrm{Cl} & -0.05436 & -1.0672 & -2.60326 \\ \mathrm{P} & 1.77746 & 0.45066 & -0.02637 \\ \mathrm{~N} & -3.43236 & -0.98336 & -0.32308 \\ \mathrm{~N} & -2.65277 & -2.95215 & -0.72295 \\ \mathrm{C} & -2.25708 & -1.68708 & -0.38733 \\ \mathrm{C} & -4.52415 & -1.80021 & -0.60807 \\ \mathrm{H} & -5.53058 & -1.41303 & -0.62024 \\ \mathrm{C} & -4.03284 & -3.0359 & -0.8508 \\ \mathrm{H} & -4.52827 & -3.95893 & -1.10919 \\ \mathrm{C} & -3.61228 & 0.43004 & -0.08001 \\ \mathrm{C} & -3.99685 & 0.85566 & 1.2044 \\ \mathrm{C} & -4.25141 & 2.21898 & 1.38779 \\ \mathrm{H} & -4.54426 & 2.56409 & 2.37676 \\ \mathrm{C} & -4.15874 & 3.14032 & 0.34058 \\ \mathrm{C} & -3.81752 & 2.66664 & -0.93073 \\ \mathrm{H} & -3.78226 & 3.36145 & -1.76706 \\ \mathrm{C} & -3.54748 & 1.31466 & -1.17275 \\ \mathrm{C} & -4.1645 & -0.11189 & 2.34956 \\ \mathrm{H} & -4.54768 & 0.40632 & 3.23368 \\ \mathrm{H} & -3.20415 & -0.57091 & 2.60676 \\ \mathrm{H} & -4.86632 & -0.91703 & 2.10022 \\ \mathrm{C} & -4.42219 & 4.60965 & 0.57466 \\ \mathrm{H} & -5.08742 & 4.76617 & 1.43026 \\ \mathrm{H} & -4.87865 & 5.08042 & -0.30293 \\ \mathrm{H} & -3.48886 & 5.14943 & 0.78285 \\ \mathrm{C} & -3.26284 & 0.83293 & -2.57596 \\ \mathrm{H} & -4.00504 & 0.09089 & -2.89695 \\ \mathrm{H} & -2.28086 & 0.35517 & -2.66711 \\ \mathrm{H} & -3.30303 & 1.66955 & -3.28006 \\ \mathrm{C} & -1.7811 & -4.11137 & -0.92959 \\ \mathrm{H} & -0.85966 & -3.74593 & -1.38391 \\ \mathrm{H} & -2.27176 & -4.74698 & -1.67416 \\ \mathrm{C} & -1.52506 & -4.916 & 0.35442 \\ \mathrm{H} & -0.86283 & -5.75177 & 0.08689 \\ \mathrm{H} & -2.47214 & -5.36576 & 0.68317 \\ \mathrm{C} & -0.92703 & -4.11947 & 1.5218 \\ \mathrm{H} & -0.85763 & -4.78281 & 2.39334 \\ \mathrm{H} & -1.59173 & -3.29789 & 1.8047 \\ \mathrm{C} & 0.47778 & -3.53209 & 1.25895 \\ \mathrm{H} & & -3.00037 & 2.17148 \\ \mathrm{C} & -.1915 & -4.35407 & 1.09461 \\ \mathrm{H} & -2.56742 & 0.10093\end{array}$




\begin{tabular}{|c|c|c|c|}
\hline $\mathrm{H}$ & 1.19982 & -2.89806 & -0.72668 \\
\hline $\mathrm{C}$ & 3.00357 & 0.25064 & -1.45117 \\
\hline $\mathrm{H}$ & 2.37153 & 0.50789 & -2.31103 \\
\hline $\mathrm{C}$ & 3.46952 & -1.1993 & -1.70579 \\
\hline $\mathrm{H}$ & 4.08703 & -1.5571 & -0.87235 \\
\hline $\mathrm{H}$ & 2.59868 & -1.85318 & -1.78198 \\
\hline $\mathrm{C}$ & 4.28077 & -1.29348 & -3.01068 \\
\hline $\mathrm{H}$ & 4.62415 & -2.32613 & -3.15569 \\
\hline $\mathrm{H}$ & 3.61702 & -1.06315 & -3.85602 \\
\hline $\mathrm{C}$ & 5.47434 & -0.32878 & -3.01962 \\
\hline $\mathrm{H}$ & 6.00354 & -0.38295 & -3.97979 \\
\hline $\mathrm{H}$ & 6.19611 & -0.63421 & -2.24688 \\
\hline $\mathrm{C}$ & 5.0198 & 1.11065 & -2.74275 \\
\hline $\mathrm{H}$ & 4.39372 & 1.46127 & -3.5763 \\
\hline $\mathrm{H}$ & 5.88522 & 1.78475 & -2.69565 \\
\hline $\mathrm{C}$ & 4.21574 & 1.20745 & -1.43324 \\
\hline $\mathrm{H}$ & 4.87625 & 0.94815 & -0.59498 \\
\hline $\mathrm{H}$ & 3.90139 & 2.24542 & -1.2755 \\
\hline $\mathrm{C}$ & 1.3051 & 2.27408 & -0.15794 \\
\hline $\mathrm{H}$ & 2.23693 & 2.83544 & -0.0025 \\
\hline $\mathrm{C}$ & 0.75599 & 2.67344 & -1.54519 \\
\hline $\mathrm{H}$ & 1.45432 & 2.39708 & -2.34172 \\
\hline $\mathrm{H}$ & -0.17205 & 2.12309 & -1.74708 \\
\hline $\mathrm{C}$ & 0.49206 & 4.18858 & -1.61165 \\
\hline $\mathrm{H}$ & 0.07853 & 4.44681 & -2.59523 \\
\hline $\mathrm{H}$ & 1.4504 & 4.72317 & -1.52719 \\
\hline $\mathrm{C}$ & -0.4516 & 4.65844 & -0.49595 \\
\hline $\mathrm{H}$ & -0.56905 & 5.74966 & -0.53248 \\
\hline $\mathrm{H}$ & -1.44665 & 4.22485 & -0.66447 \\
\hline $\mathrm{C}$ & 0.05374 & 4.21996 & 0.88593 \\
\hline $\mathrm{H}$ & -0.66966 & 4.4991 & 1.66295 \\
\hline $\mathrm{H}$ & 0.98615 & 4.75582 & 1.1211 \\
\hline $\mathrm{C}$ & 0.31032 & 2.70423 & 0.94287 \\
\hline $\mathrm{H}$ & -0.63688 & 2.16597 & 0.81333 \\
\hline $\mathrm{H}$ & 0.67363 & 2.42476 & 1.93702 \\
\hline $\mathrm{C}$ & 2.66743 & 0.35131 & 1.63775 \\
\hline $\mathrm{H}$ & 1.8092 & 0.3015 & 2.32141 \\
\hline $\mathrm{C}$ & 3.54723 & 1.54593 & 2.07013 \\
\hline $\mathrm{H}$ & 4.42858 & 1.6252 & 1.42079 \\
\hline $\mathrm{H}$ & 3.00369 & 2.49182 & 1.97942 \\
\hline $\mathrm{C}$ & 4.01145 & 1.37702 & 3.52974 \\
\hline $\mathrm{H}$ & 4.65166 & 2.22291 & 3.81279 \\
\hline $\mathrm{H}$ & 3.13267 & 1.41371 & 4.18961 \\
\hline $\mathrm{C}$ & 4.75288 & 0.05065 & 3.74753 \\
\hline $\mathrm{H}$ & 5.02286 & -0.06484 & 4.80512 \\
\hline $\mathrm{H}$ & 5.69767 & 0.06705 & 3.18332 \\
\hline $\mathrm{C}$ & 3.90601 & -1.14082 & 3.27799 \\
\hline $\mathrm{H}$ & 3.01824 & -1.236 & 3.91905 \\
\hline
\end{tabular}




$\begin{array}{llll}\mathrm{H} & 4.47297 & -2.07535 & 3.38157 \\ \mathrm{C} & 3.45363 & -0.96497 & 1.81814 \\ \mathrm{H} & 2.84502 & -1.8223 & 1.51136 \\ \mathrm{H} & 4.34265 & -0.95785 & 1.17293\end{array}$

UC5 Open Adduct, B3LYP/6-31G(d)//LANL2DZ (Energy = -2911.90840309 Hartrees)

$\begin{array}{llll}\mathrm{Ru} & 0.36459 & 0.64262 & -0.14244 \\ \mathrm{Cl} & 0.79265 & -0.43886 & 2.02697 \\ \mathrm{Cl} & -0.01458 & 1.09692 & -2.54302 \\ \mathrm{P} & -1.96212 & -0.34902 & -0.06576 \\ \mathrm{~N} & 3.45478 & 0.16113 & -0.49316 \\ \mathrm{~N} & 2.96846 & 2.18492 & -1.06061 \\ \mathrm{C} & 2.40759 & 1.04987 & -0.53256 \\ \mathrm{C} & 4.62288 & 0.73413 & -0.98573 \\ \mathrm{H} & 5.54527 & 0.17787 & -1.04023 \\ \mathrm{C} & 4.31645 & 2.00263 & -1.33681 \\ \mathrm{H} & 4.92665 & 2.78302 & -1.76311 \\ \mathrm{C} & 3.42692 & -1.23986 & -0.1396 \\ \mathrm{C} & 3.88762 & -1.63513 & 1.12682 \\ \mathrm{C} & 3.92341 & -3.00355 & 1.40784 \\ \mathrm{H} & 4.26834 & -3.32467 & 2.38815 \\ \mathrm{C} & 3.52645 & -3.96435 & 0.47279 \\ \mathrm{C} & 3.11286 & -3.5291 & -0.78974 \\ \mathrm{H} & 2.84014 & -4.26396 & -1.54406 \\ \mathrm{C} & 3.06269 & -2.17114 & -1.12676 \\ \mathrm{C} & 4.32913 & -0.6288 & 2.16002 \\ \mathrm{H} & 4.74698 & -1.13535 & 3.03516 \\ \mathrm{H} & 3.47331 & -0.02912 & 2.48636 \\ \mathrm{H} & 5.09124 & 0.05572 & 1.76916 \\ \mathrm{C} & 3.53758 & -5.43454 & 0.82161 \\ \mathrm{H} & 4.34147 & -5.67414 & 1.52609 \\ \mathrm{H} & 3.66796 & -6.05837 & -0.06913 \\ \mathrm{H} & 2.5926 & -5.73383 & 1.29437 \\ \mathrm{C} & 2.68891 & -1.74493 & -2.52843 \\ \mathrm{H} & 3.53399 & -1.25637 & -3.03085 \\ \mathrm{H} & 1.8591 & -1.03002 & -2.55249 \\ \mathrm{H} & 2.40591 & -2.61475 & -3.12881 \\ \mathrm{C} & 2.27768 & 3.42053 & -1.45117 \\ \mathrm{H} & 1.22305 & 3.28381 & -1.22259 \\ \mathrm{H} & 2.34977 & 3.50933 & -2.54118 \\ \mathrm{C} & -0.10355 & 2.28305 & 0.52891 \\ \mathrm{H} & -0.92073 & 2.78487 & -0.01371 \\ \mathrm{C} & -3.22002 & 0.38052 & -1.2774 \\ \mathrm{H} & & 0.13961 & -2.24042 \\ & & & \\ & & & \end{array}$




\begin{tabular}{|c|c|c|c|}
\hline $\mathrm{C}$ & -3.32286 & 1.92117 & -1.25036 \\
\hline $\mathrm{H}$ & -3.73827 & 2.26414 & -0.29397 \\
\hline $\mathrm{H}$ & -2.32442 & 2.3507 & -1.35667 \\
\hline $\mathrm{C}$ & -4.20745 & 2.43678 & -2.40026 \\
\hline $\mathrm{H}$ & -4.29137 & 3.52967 & -2.33714 \\
\hline $\mathrm{H}$ & -3.70822 & 2.21641 & -3.35458 \\
\hline $\mathrm{C}$ & -5.60058 & 1.79338 & -2.39446 \\
\hline $\mathrm{H}$ & -6.18814 & 2.145 & -3.25239 \\
\hline $\mathrm{H}$ & -6.14516 & 2.10893 & -1.49158 \\
\hline $\mathrm{C}$ & -5.49687 & 0.26243 & -2.41032 \\
\hline $\mathrm{H}$ & -5.05675 & -0.06292 & -3.36436 \\
\hline $\mathrm{H}$ & -6.49525 & -0.19119 & -2.35483 \\
\hline $\mathrm{C}$ & -4.6274 & -0.25468 & -1.2499 \\
\hline $\mathrm{H}$ & -5.12441 & -0.00618 & -0.3028 \\
\hline $\mathrm{H}$ & -4.56984 & -1.34823 & -1.29661 \\
\hline $\mathrm{C}$ & -1.92564 & -2.16671 & -0.59145 \\
\hline $\mathrm{H}$ & -2.94779 & -2.53966 & -0.43609 \\
\hline $\mathrm{C}$ & -1.58447 & -2.37394 & -2.08393 \\
\hline $\mathrm{H}$ & -2.27199 & -1.81476 & -2.72662 \\
\hline $\mathrm{H}$ & -0.58336 & -1.9771 & -2.29352 \\
\hline $\mathrm{C}$ & -1.64743 & -3.86426 & -2.46451 \\
\hline $\mathrm{H}$ & -1.37254 & -3.98415 & -3.5205 \\
\hline $\mathrm{H}$ & -2.68633 & -4.21569 & -2.37177 \\
\hline $\mathrm{C}$ & -0.74279 & -4.72645 & -1.57411 \\
\hline $\mathrm{H}$ & -0.85082 & -5.78758 & -1.83457 \\
\hline $\mathrm{H}$ & 0.30734 & -4.46047 & -1.76097 \\
\hline $\mathrm{C}$ & -1.05531 & -4.50252 & -0.08838 \\
\hline $\mathrm{H}$ & -0.35924 & -5.07465 & 0.53868 \\
\hline $\mathrm{H}$ & -2.06307 & -4.88593 & 0.13368 \\
\hline $\mathrm{C}$ & -0.97866 & -3.01316 & 0.28803 \\
\hline $\mathrm{H}$ & 0.05176 & -2.6589 & 0.16639 \\
\hline $\mathrm{H}$ & -1.20749 & -2.88571 & 1.35088 \\
\hline $\mathrm{C}$ & -2.69427 & -0.41846 & 1.67645 \\
\hline $\mathrm{H}$ & -1.80175 & -0.68807 & 2.25694 \\
\hline $\mathrm{C}$ & -3.7863 & -1.46966 & 1.97663 \\
\hline $\mathrm{H}$ & -4.70501 & -1.23555 & 1.42415 \\
\hline $\mathrm{H}$ & -3.47223 & -2.46803 & 1.65549 \\
\hline $\mathrm{C}$ & -4.10345 & -1.50772 & 3.48404 \\
\hline $\mathrm{H}$ & -4.89447 & -2.24466 & 3.67581 \\
\hline $\mathrm{H}$ & -3.21275 & -1.85438 & 4.02768 \\
\hline $\mathrm{C}$ & -4.51774 & -0.12945 & 4.01803 \\
\hline $\mathrm{H}$ & -4.68831 & -0.1764 & 5.1013 \\
\hline $\mathrm{H}$ & -5.47627 & 0.16226 & 3.56262 \\
\hline $\mathrm{C}$ & -3.45879 & 0.93226 & 3.68889 \\
\hline $\mathrm{H}$ & -2.53348 & 0.71027 & 4.23905 \\
\hline $\mathrm{H}$ & -3.79575 & 1.92259 & 4.02274 \\
\hline $\mathrm{C}$ & -3.14843 & 0.96742 & 2.18241 \\
\hline $\mathrm{H}$ & -2.37686 & 1.71822 & 1.9803 \\
\hline
\end{tabular}




$\begin{array}{llll}\mathrm{H} & -4.05174 & 1.28422 & 1.64299 \\ \mathrm{C} & 0.35114 & 3.14035 & 1.68177 \\ \mathrm{H} & 0.40286 & 4.1734 & 1.29056 \\ \mathrm{H} & -0.50445 & 3.16852 & 2.38156 \\ \mathrm{C} & 1.62777 & 2.82691 & 2.46928 \\ \mathrm{H} & 2.47354 & 2.74504 & 1.77661 \\ \mathrm{H} & 1.52432 & 1.84703 & 2.93936 \\ \mathrm{C} & 1.92178 & 3.90064 & 3.52345 \\ \mathrm{H} & 2.83347 & 3.66368 & 4.08355 \\ \mathrm{H} & 1.10139 & 3.98291 & 4.24782 \\ \mathrm{H} & 2.05885 & 4.89153 & 3.06966 \\ \mathrm{C} & 2.84176 & 4.67448 & -0.7734 \\ \mathrm{H} & 2.77665 & 4.561 & 0.31491 \\ \mathrm{H} & 3.90813 & 4.78561 & -1.01046 \\ \mathrm{C} & 2.08962 & 5.93346 & -1.21925 \\ \mathrm{H} & 2.50012 & 6.82695 & -0.73723 \\ \mathrm{H} & 2.15925 & 6.07632 & -2.3043 \\ \mathrm{H} & 1.02534 & 5.87328 & -0.96179\end{array}$

CB6, B3LYP/6-31G(d)//LANL2DZ (Energy = -3603.06176552 Hartrees)

$\begin{array}{llll}\mathrm{Cl} & 0.55356 & -0.24187 & -2.25576 \\ \mathrm{Cl} & 1.34586 & -0.63501 & 2.63886 \\ \mathrm{Ru} & 0.8127 & -0.40582 & 0.20805 \\ \mathrm{P} & 2.06364 & 1.83378 & 0.15356 \\ \mathrm{C} & 1.05235 & 3.42738 & 0.18425 \\ \mathrm{H} & 1.78752 & 4.24415 & 0.19008 \\ \mathrm{C} & 0.20867 & 3.554 & 1.4706 \\ \mathrm{H} & 0.83957 & 3.46354 & 2.36202 \\ \mathrm{H} & -0.50805 & 2.72687 & 1.51336 \\ \mathrm{C} & -0.55596 & 4.88887 & 1.51189 \\ \mathrm{H} & -1.16762 & 4.93373 & 2.42243 \\ \mathrm{H} & 0.16355 & 5.71908 & 1.57873 \\ \mathrm{C} & -1.43353 & 5.07691 & 0.26653 \\ \mathrm{H} & -2.22762 & 4.31682 & 0.26511 \\ \mathrm{H} & -1.93218 & 6.0547 & 0.29616 \\ \mathrm{C} & -0.60163 & 4.9428 & -1.01654 \\ \mathrm{H} & 0.11383 & 5.77756 & -1.07203 \\ \mathrm{H} & -1.24683 & 5.02628 & -1.90051 \\ \mathrm{C} & 0.16776 & 3.61061 & -1.06754 \\ \mathrm{H} & 0.7756 & 3.58132 & -1.97832 \\ \mathrm{H} & -0.54095 & 2.77948 & -1.14147 \\ \mathrm{C} & 3.04787 & 2.00476 & -1.44719 \\ \mathrm{H} & 2.23853 & 1.98176 & -2.18911 \\ \mathrm{C} & 3.84867 & 3.30914 & -1.64656 \\ \mathrm{H} & 3.2353 & 4.19248 & -1.43492 \\ \mathrm{H} & 4.69274 & 3.33629 & -0.9444\end{array}$




\begin{tabular}{|c|c|c|c|}
\hline $\mathrm{C}$ & 4.39461 & 3.40035 & -3.08405 \\
\hline $\mathrm{H}$ & 3.54936 & 3.47483 & -3.78383 \\
\hline $\mathrm{H}$ & 4.97998 & 4.32209 & -3.20064 \\
\hline $\mathrm{C}$ & 5.24661 & 2.17545 & -3.44688 \\
\hline $\mathrm{H}$ & 6.15847 & 2.17674 & -2.83035 \\
\hline $\mathrm{H}$ & 5.57672 & 2.2393 & -4.49203 \\
\hline $\mathrm{C}$ & 4.47459 & 0.87014 & -3.20535 \\
\hline $\mathrm{H}$ & 5.11673 & 0.00381 & -3.41118 \\
\hline $\mathrm{H}$ & 3.62848 & 0.80626 & -3.90388 \\
\hline $\mathrm{C}$ & 3.9375 & 0.78362 & -1.76667 \\
\hline $\mathrm{H}$ & 4.78515 & 0.73809 & -1.07281 \\
\hline $\mathrm{H}$ & 3.3687 & -0.14221 & -1.6475 \\
\hline $\mathrm{C}$ & 3.20028 & 2.03933 & 1.65048 \\
\hline $\mathrm{H}$ & 2.52155 & 1.76141 & 2.46776 \\
\hline $\mathrm{C}$ & 3.76294 & 3.4461 & 1.95406 \\
\hline $\mathrm{H}$ & 4.47678 & 3.74425 & 1.17519 \\
\hline $\mathrm{H}$ & 2.97231 & 4.20343 & 1.9602 \\
\hline $\mathrm{C}$ & 4.47438 & 3.45976 & 3.32116 \\
\hline $\mathrm{H}$ & 4.8859 & 4.45974 & 3.51263 \\
\hline $\mathrm{H}$ & 3.73215 & 3.26982 & 4.11018 \\
\hline $\mathrm{C}$ & 5.58286 & 2.40082 & 3.40475 \\
\hline $\mathrm{H}$ & 6.02868 & 2.39745 & 4.408 \\
\hline $\mathrm{H}$ & 6.38963 & 2.66781 & 2.70516 \\
\hline $\mathrm{C}$ & 5.04705 & 1.00654 & 3.04921 \\
\hline $\mathrm{H}$ & 4.32969 & 0.67592 & 3.81256 \\
\hline $\mathrm{H}$ & 5.8663 & 0.27512 & 3.0473 \\
\hline $\mathrm{C}$ & 4.34768 & 1.0061 & 1.67994 \\
\hline $\mathrm{H}$ & 3.96663 & 0.00484 & 1.4595 \\
\hline $\mathrm{H}$ & 5.08758 & 1.25518 & 0.90776 \\
\hline $\mathrm{C}$ & 0.47213 & -2.47259 & 0.23261 \\
\hline $\mathrm{C}$ & 1.10976 & -4.75238 & 0.37726 \\
\hline $\mathrm{H}$ & 1.63871 & -5.09101 & 1.27491 \\
\hline $\mathrm{H}$ & 1.37994 & -5.41725 & -0.44925 \\
\hline $\mathrm{C}$ & -0.40186 & -4.64362 & 0.59378 \\
\hline $\mathrm{H}$ & -0.98124 & -5.12957 & -0.20022 \\
\hline $\mathrm{N}$ & 1.48851 & -3.36033 & 0.06145 \\
\hline $\mathrm{C}$ & 2.83264 & -3.11037 & -0.39133 \\
\hline C & 3.09331 & -3.15007 & -1.77943 \\
\hline $\mathrm{C}$ & 4.4122 & -2.98301 & -2.21243 \\
\hline $\mathrm{H}$ & 4.61618 & -3.00836 & -3.28076 \\
\hline $\mathrm{C}$ & 5.47104 & -2.80376 & -1.31647 \\
\hline $\mathrm{C}$ & 5.1883 & -2.8414 & 0.05052 \\
\hline $\mathrm{H}$ & 6.00373 & -2.75425 & 0.76553 \\
\hline $\mathrm{C}$ & 3.88797 & -3.01847 & 0.54046 \\
\hline $\mathrm{C}$ & 2.00163 & -3.40795 & -2.78901 \\
\hline $\mathrm{H}$ & 1.40843 & -4.29297 & -2.52797 \\
\hline $\mathrm{H}$ & 2.43391 & -3.57788 & -3.7800 \\
\hline $\mathrm{H}$ & 1.31727 & -2.55626 & -2.8550 \\
\hline
\end{tabular}




\begin{tabular}{|c|c|c|c|}
\hline $\mathrm{C}$ & 6.88027 & -2.58548 & -1.81557 \\
\hline $\mathrm{H}$ & 7.07397 & -3.15389 & -2.73204 \\
\hline $\mathrm{H}$ & 7.62221 & -2.88283 & -1.067 \\
\hline $\mathrm{H}$ & 7.05685 & -1.52686 & -2.04847 \\
\hline $\mathrm{C}$ & 3.67483 & -3.18957 & 2.0254 \\
\hline $\mathrm{H}$ & 3.5783 & -4.25465 & 2.28085 \\
\hline $\mathrm{H}$ & 2.78443 & -2.66692 & 2.38108 \\
\hline $\mathrm{H}$ & 4.53533 & -2.80554 & 2.58225 \\
\hline $\mathrm{N}$ & -0.63324 & -3.18315 & 0.56433 \\
\hline $\mathrm{C}$ & -1.99539 & -2.7427 & 0.66925 \\
\hline $\mathrm{C}$ & -2.7678 & -2.63491 & -0.50022 \\
\hline $\mathrm{C}$ & -4.12417 & -2.30806 & -0.3885 \\
\hline $\mathrm{H}$ & -4.71127 & -2.21712 & -1.29466 \\
\hline $\mathrm{C}$ & -4.70456 & -2.09764 & 0.86497 \\
\hline $\mathrm{C}$ & -3.92853 & -2.2452 & 2.02164 \\
\hline $\mathrm{H}$ & -4.40371 & -2.09094 & 2.98584 \\
\hline $\mathrm{C}$ & -2.5757 & -2.57396 & 1.94473 \\
\hline $\mathrm{C}$ & -2.16606 & -2.85159 & -1.86827 \\
\hline $\mathrm{H}$ & -1.40144 & -2.09972 & -2.09473 \\
\hline $\mathrm{H}$ & -2.93862 & -2.79588 & -2.64137 \\
\hline $\mathrm{H}$ & -1.68559 & -3.83431 & -1.95309 \\
\hline $\mathrm{C}$ & -1.76345 & -2.74241 & 3.20702 \\
\hline $\mathrm{H}$ & -2.34135 & -2.42608 & 4.08089 \\
\hline $\mathrm{H}$ & -0.83536 & -2.16182 & 3.17358 \\
\hline $\mathrm{H}$ & -1.48396 & -3.79292 & 3.36666 \\
\hline $\mathrm{O}$ & -6.0124 & -1.75458 & 1.05809 \\
\hline $\mathrm{C}$ & -6.79704 & -1.40192 & -0.0821 \\
\hline $\mathrm{H}$ & -6.97358 & -2.28792 & -0.71061 \\
\hline $\mathrm{H}$ & -6.25096 & -0.66739 & -0.68514 \\
\hline $\mathrm{C}$ & -8.11862 & -0.82995 & 0.41952 \\
\hline $\mathrm{H}$ & -7.90174 & -0.04138 & 1.14871 \\
\hline $\mathrm{H}$ & -8.64944 & -1.61291 & 0.97553 \\
\hline $\mathrm{C}$ & -9.03675 & -0.30757 & -0.70314 \\
\hline $\mathrm{H}$ & -9.33309 & -1.1608 & -1.32989 \\
\hline $\mathrm{H}$ & -9.96459 & 0.05486 & -0.2393 \\
\hline $\mathrm{C}$ & -8.49548 & 0.79041 & -1.64604 \\
\hline $\mathrm{H}$ & -9.2471 & 0.92219 & -2.43507 \\
\hline $\mathrm{H}$ & -7.58948 & 0.44848 & -2.16006 \\
\hline $\mathrm{C}$ & -8.22329 & 2.1814 & -1.03795 \\
\hline $\mathrm{H}$ & -8.21251 & 2.92206 & -1.84739 \\
\hline $\mathrm{H}$ & -9.04443 & 2.46809 & -0.36569 \\
\hline $\mathrm{C}$ & -6.91625 & 2.32704 & -0.26127 \\
\hline $\mathrm{H}$ & -6.81837 & 3.34426 & 0.14322 \\
\hline $\mathrm{H}$ & -6.8764 & 1.62685 & 0.57741 \\
\hline $\mathrm{O}$ & -5.82968 & 2.0689 & -1.15814 \\
\hline $\mathrm{C}$ & -0.85363 & 0.22205 & 0.75959 \\
\hline $\mathrm{H}$ & -0.91648 & 0.22288 & 1.85508 \\
\hline $\mathrm{C}$ & -2.08622 & 0.74886 & 0.1861 \\
\hline
\end{tabular}




$\begin{array}{llll}\mathrm{C} & -2.41702 & 0.83963 & -1.18879 \\ \mathrm{H} & -1.68615 & 0.52099 & -1.92225 \\ \mathrm{C} & -3.6534 & 1.30887 & -1.59738 \\ \mathrm{H} & -3.91422 & 1.35345 & -2.6506 \\ \mathrm{C} & -4.61802 & 1.71024 & -0.65383 \\ \mathrm{C} & -4.29739 & 1.69082 & 0.71081 \\ \mathrm{H} & -5.00122 & 2.03046 & 1.46123 \\ \mathrm{C} & -3.05733 & 1.20113 & 1.10834 \\ \mathrm{H} & -2.83115 & 1.15983 & 2.17049 \\ \mathrm{H} & -0.72628 & -5.05999 & 1.55182\end{array}$

CB6 Open Adduct, B3LYP/6-31G(d)//LANL2DZ (Energy = -3682.89846429 Hartrees)

\begin{tabular}{llll}
$\mathrm{Cl}$ & 0.87458 & -0.08871 & -2.25767 \\
$\mathrm{Cl}$ & 1.68856 & -0.94728 & 2.56927 \\
$\mathrm{Ru}$ & 1.13054 & -0.49918 & 0.17619 \\
$\mathrm{P}$ & 2.18412 & 1.83976 & 0.39652 \\
$\mathrm{C}$ & 1.03936 & 3.3177 & 0.65784 \\
$\mathrm{H}$ & 1.70101 & 4.18623 & 0.78148 \\
$\mathrm{C}$ & 0.20548 & 3.18254 & 1.94978 \\
$\mathrm{H}$ & 0.85432 & 3.0176 & 2.81743 \\
$\mathrm{H}$ & -0.43828 & 2.29931 & 1.87235 \\
$\mathrm{C}$ & -0.6677 & 4.42741 & 2.18659 \\
$\mathrm{H}$ & -1.26934 & 4.28695 & 3.09408 \\
$\mathrm{H}$ & -0.01952 & 5.29696 & 2.37404 \\
$\mathrm{C}$ & -1.5739 & 4.7205 & 0.98267 \\
$\mathrm{H}$ & -2.30203 & 3.90459 & 0.87085 \\
$\mathrm{H}$ & -2.15148 & 5.6383 & 1.15535 \\
$\mathrm{C}$ & -0.75119 & 4.84495 & -0.30737 \\
$\mathrm{H}$ & -0.10855 & 5.73626 & -0.24156 \\
$\mathrm{H}$ & -1.41317 & 5.00119 & -1.16886 \\
$\mathrm{C}$ & 0.12571 & 3.60398 & -0.5529 \\
$\mathrm{H}$ & 0.72128 & 3.7584 & -1.45923 \\
$\mathrm{H}$ & -0.51338 & 2.73632 & -0.74533 \\
$\mathrm{C}$ & 3.11038 & 2.31076 & -1.17869 \\
$\mathrm{H}$ & 2.29126 & 2.29633 & -1.91027 \\
$\mathrm{C}$ & 3.76973 & 3.70588 & -1.21951 \\
$\mathrm{H}$ & 3.07565 & 4.48766 & -0.89046 \\
$\mathrm{H}$ & 4.62323 & 3.732 & -0.52883 \\
$\mathrm{C}$ & 4.26855 & 4.03253 & -2.63982 \\
$\mathrm{H}$ & 3.40316 & 4.10599 & -3.31471 \\
$\mathrm{H}$ & 4.75422 & 5.01742 & -2.64501 \\
$\mathrm{C}$ & 5.23144 & 2.95807 & -3.16551 \\
$\mathrm{H}$ & 6.15388 & 2.97776 & -2.56521 \\
$\mathrm{H}$ & 5.52656 & 3.18597 & -4.19817 \\
& & & \\
\hline & & &
\end{tabular}




\begin{tabular}{|c|c|c|c|}
\hline C & 4.60334 & 1.55927 & -3.0815 \\
\hline $\mathrm{H}$ & 5.32574 & 0.79729 & -3.40236 \\
\hline $\mathrm{H}$ & 3.75124 & 1.49374 & -3.77247 \\
\hline C & 4.11379 & 1.23917 & -1.65899 \\
\hline $\mathrm{H}$ & 4.97884 & 1.20049 & -0.98645 \\
\hline $\mathrm{H}$ & 3.64836 & 0.25022 & -1.65021 \\
\hline C & 3.33387 & 1.94662 & 1.89482 \\
\hline $\mathrm{H}$ & 2.69656 & 1.51296 & 2.67665 \\
\hline C & 3.7879 & 3.3443 & 2.3713 \\
\hline $\mathrm{H}$ & 4.45769 & 3.79831 & 1.62939 \\
\hline $\mathrm{H}$ & 2.93858 & 4.02586 & 2.48502 \\
\hline $\mathrm{C}$ & 4.5255 & 3.24239 & 3.72073 \\
\hline $\mathrm{H}$ & 4.86061 & 4.24018 & 4.03395 \\
\hline $\mathrm{H}$ & 3.81778 & 2.89443 & 4.48724 \\
\hline C & 5.71579 & 2.27482 & 3.65619 \\
\hline $\mathrm{H}$ & 6.18186 & 2.18111 & 4.64577 \\
\hline $\mathrm{H}$ & 6.48377 & 2.69224 & 2.98719 \\
\hline C & 5.28395 & 0.89785 & 3.13194 \\
\hline $\mathrm{H}$ & 4.61026 & 0.41746 & 3.85445 \\
\hline $\mathrm{H}$ & 6.15785 & 0.24068 & 3.02885 \\
\hline $\mathrm{C}$ & 4.55945 & 1.01423 & 1.78079 \\
\hline $\mathrm{H}$ & 4.25402 & 0.02152 & 1.43765 \\
\hline $\mathrm{H}$ & 5.26215 & 1.41703 & 1.03954 \\
\hline C & 0.97217 & -2.56918 & -0.0516 \\
\hline C & 1.79866 & -4.78419 & -0.22091 \\
\hline $\mathrm{H}$ & 2.36311 & -5.17809 & 0.63196 \\
\hline $\mathrm{H}$ & 2.1178 & -5.32094 & -1.1194 \\
\hline C & 0.28619 & -4.83383 & 0.00593 \\
\hline $\mathrm{H}$ & -0.25945 & -5.23262 & -0.85794 \\
\hline $\mathrm{N}$ & 2.05015 & -3.33653 & -0.36834 \\
\hline C & 3.35865 & -2.91317 & -0.79601 \\
\hline C & 3.60127 & -2.77592 & -2.18105 \\
\hline $\mathrm{C}$ & 4.89076 & -2.43315 & -2.59822 \\
\hline $\mathrm{H}$ & 5.08026 & -2.32031 & -3.66354 \\
\hline $\mathrm{C}$ & 5.94036 & -2.24977 & -1.69204 \\
\hline C & 5.68319 & -2.46498 & -0.33657 \\
\hline $\mathrm{H}$ & 6.49699 & -2.37753 & 0.38024 \\
\hline C & 4.41335 & -2.82098 & 0.13616 \\
\hline C & 2.52481 & -3.03129 & -3.20751 \\
\hline $\mathrm{H}$ & 2.05169 & -4.01065 & -3.06315 \\
\hline $\mathrm{H}$ & 2.95004 & -3.01408 & -4.21581 \\
\hline $\mathrm{H}$ & 1.74109 & -2.26959 & -3.15309 \\
\hline C & 7.31386 & -1.83999 & -2.16983 \\
\hline $\mathrm{H}$ & 7.55239 & -2.2905 & -3.13975 \\
\hline $\mathrm{H}$ & 8.09151 & -2.13417 & -1.45713 \\
\hline $\mathrm{H}$ & 7.37947 & -0.75086 & -2.29464 \\
\hline $\mathrm{C}$ & 4.23914 & -3.17352 & 1.59409 \\
\hline $\mathrm{H}$ & 4.22004 & -4.26461 & 1.72787 \\
\hline
\end{tabular}




\begin{tabular}{|c|c|c|c|}
\hline $\mathrm{H}$ & 3.32154 & -2.75951 & 2.0179 \\
\hline $\mathrm{H}$ & 5.08147 & -2.79553 & 2.18197 \\
\hline $\mathrm{N}$ & -0.05945 & -3.41006 & 0.20615 \\
\hline $\mathrm{C}$ & -1.44199 & -3.10334 & 0.43821 \\
\hline C & -2.29104 & -2.89258 & -0.66226 \\
\hline C & -3.65981 & -2.70315 & -0.4364 \\
\hline $\mathrm{H}$ & -4.30627 & -2.52503 & -1.28759 \\
\hline $\mathrm{C}$ & -4.1752 & -2.73162 & 0.86218 \\
\hline C & -3.31865 & -2.96886 & 1.94476 \\
\hline $\mathrm{H}$ & -3.7456 & -3.00257 & 2.94266 \\
\hline $\mathrm{C}$ & -1.95278 & -3.16668 & 1.75299 \\
\hline C & -1.75702 & -2.85766 & -2.07451 \\
\hline $\mathrm{H}$ & -1.0282 & -2.05094 & -2.2109 \\
\hline $\mathrm{H}$ & -2.57095 & -2.70658 & -2.79008 \\
\hline $\mathrm{H}$ & -1.25324 & -3.79506 & -2.34272 \\
\hline $\mathrm{C}$ & -1.05641 & -3.45062 & 2.93452 \\
\hline $\mathrm{H}$ & -1.61211 & -3.34599 & 3.87145 \\
\hline $\mathrm{H}$ & -0.19611 & -2.77345 & 2.96172 \\
\hline $\mathrm{H}$ & -0.66332 & -4.47598 & 2.90265 \\
\hline $\mathrm{O}$ & -5.49047 & -2.55137 & 1.17585 \\
\hline $\mathrm{C}$ & -6.42189 & -2.31007 & 0.12334 \\
\hline $\mathrm{H}$ & -6.41839 & -3.15423 & -0.58215 \\
\hline $\mathrm{H}$ & -6.13293 & -1.40646 & -0.4328 \\
\hline $\mathrm{C}$ & -7.79939 & -2.14227 & 0.75155 \\
\hline $\mathrm{H}$ & -7.75945 & -1.31356 & 1.47133 \\
\hline $\mathrm{H}$ & -8.03538 & -3.04564 & 1.32885 \\
\hline $\mathrm{C}$ & -8.89527 & -1.88241 & -0.29042 \\
\hline $\mathrm{H}$ & -8.91792 & -2.71138 & -1.01171 \\
\hline $\mathrm{C}$ & -7.79712 & 2.79743 & -0.85293 \\
\hline $\mathrm{H}$ & -8.6406 & 2.80095 & -0.14892 \\
\hline $\mathrm{C}$ & -6.59932 & 2.24628 & -0.0861 \\
\hline $\mathrm{H}$ & -6.2338 & 2.98117 & 0.64601 \\
\hline $\mathrm{H}$ & -6.88827 & 1.33863 & 0.46309 \\
\hline $\mathrm{O}$ & -5.55368 & 1.92579 & -1.00802 \\
\hline $\mathrm{C}$ & -0.59655 & -0.11741 & 0.77005 \\
\hline $\mathrm{H}$ & -0.68073 & -0.29264 & 1.84988 \\
\hline $\mathrm{C}$ & -1.85408 & 0.39866 & 0.24119 \\
\hline $\mathrm{C}$ & -2.14511 & 0.69305 & -1.11439 \\
\hline $\mathrm{H}$ & -1.3776 & 0.52452 & -1.85994 \\
\hline $\mathrm{C}$ & -3.3785 & 1.1944 & -1.48988 \\
\hline $\mathrm{H}$ & -3.59794 & 1.41907 & -2.5294 \\
\hline $\mathrm{C}$ & -4.38045 & 1.43256 & -0.52995 \\
\hline $\mathrm{C}$ & -4.12439 & 1.15133 & 0.81968 \\
\hline $\mathrm{H}$ & -4.87628 & 1.31792 & 1.582 \\
\hline $\mathrm{C}$ & -2.88197 & 0.63681 & 1.18194 \\
\hline $\mathrm{H}$ & -2.69769 & 0.41122 & 2.22876 \\
\hline $\mathrm{H}$ & 0.00514 & -5.42135 & 0.88441 \\
\hline $\mathrm{C}$ & -10.28368 & -1.71546 & 0.33622 \\
\hline
\end{tabular}




$\begin{array}{llll}\mathrm{H} & -10.57775 & -2.61435 & 0.89138 \\ \mathrm{H} & -11.04604 & -1.53112 & -0.4289 \\ \mathrm{H} & -10.30344 & -0.87251 & 1.0378 \\ \mathrm{C} & -6.69192 & 4.32756 & -2.63909 \\ \mathrm{H} & -5.66685 & 4.05293 & -2.37769 \\ \mathrm{H} & -7.0126 & 53.66564 & -3.45292 \\ \mathrm{H} & -6.6846 & 5.35298 & -3.02661 \\ \mathrm{H} & -8.64471 & -0.98204 & -0.8696 \\ \mathrm{C} & -7.63591 & 4.21285 & -1.43584 \\ \mathrm{H} & -8.63195 & 4.56878 & -1.73157 \\ \mathrm{H} & -7.30025 & 4.8897 & -0.63668 \\ \mathrm{H} & -8.05905 & 2.08681 & -1.64858\end{array}$

Ethane, B3LYP/6-31G(d) (Energy = -79.8127393795 Hartrees)

$\begin{array}{llll}\mathrm{C} & 0 . & 0 . & 0.76766 \\ \mathrm{H} & 0 . & 1.0218 & 1.16583 \\ \mathrm{H} & -0.88491 & -0.5109 & 1.16583 \\ \mathrm{H} & 0.88491 & -0.5109 & 1.16583 \\ \mathrm{C} & 0 . & 0 . & -0.76766 \\ \mathrm{H} & -0.88491 & 0.5109 & -1.16583 \\ \mathrm{H} & 0 . & -1.0218 & -1.16583 \\ \mathrm{H} & 0.88491 & 0.5109 & -1.16583\end{array}$

